$$
\begin{aligned}
& \text { Universidade de São Paulo } \\
& \text { Instituto de Física de São Carlos }
\end{aligned}
$$

Gabriel Luchini Martins

\title{
Hidden symmetries in gauge theories \& quasi-integrablility
}



Gabriel Luchini Martins

\section{Hidden symmetries in gauge theories \& quasi-integrablility}

Tese apresentada ao Programa de PósGraduação em Física do Instituto de Física de São Carlos da Universidade de São Paulo, para obtenção do título de doutor em Ciências.

Área de Concentração: Física Básica

Orientador: Prof. Dr. Luiz Agostinho Ferreira

Versão Corrigida

(versão original disponível na Unidade que aloja o Programa)

São Carlos 
AUTORIZO A REPRODUÇÃO E DIVULGAÇÃO TOTAL OU PARCIAL DESTE TRABALHO, POR QUALQÜER MEIO CONVENCIONAL OU ELETRÔNICO PARA FINS DE ESTUDO E PESQUISA, DESDE QUE CITADA A FONTE.

Ficha catalográfica elaborada pelo Serviço de Biblioteca e Informação do IFSC, com os dados fornecidos pelo(a) autor(a)

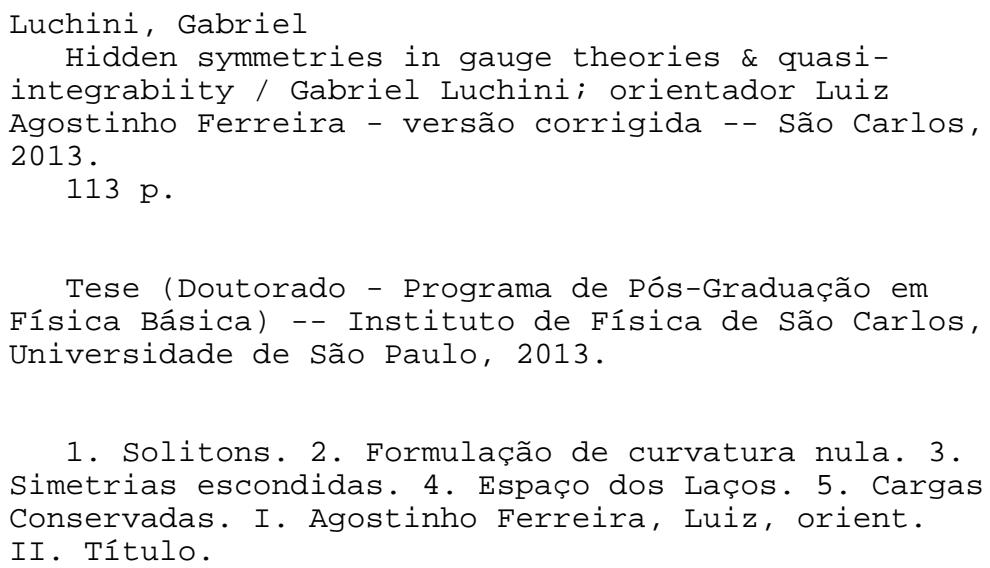




\section{ACKNOWLEDGEMENTS}

I would like to express my deep gratitude to Clisthenis P. Constantinidis for his companionship since the beginning of my studies in physics; from the first year of my graduation as a professor, as my supervisor during my masters and as a very good friend always. I finish my PhD studies in São Carlos thanks to his many good advises, including the one stating I should come to work with Luiz Agostinho.

I wish to thank my friends in Vitória, Zé, Ulysses dS, Ivanzito (and all respective ladies) for standing by me in every new step I make. I also want to thank Massayuki for having listened to me during these 4 years. I could say more, but I think I've said enough. I would also like to thank Ritinha for her friendship, and Yvoninha and Mariana for all the help they gave me since I came to São Carlos. I wish to express my gratitude for the staff in the Institute and in particular to Silvio, who can still be very patient with my requests!

This work is a consequence of hundreds knocks on Luiz's door, who luckily moved to another room a little bit more distant from my office during my second year as a student. I learned with him many valuable lessons, but two of them are very special: first, that an example is much better than a thousand theorems, and the other one is that research is something that must be done for yourself, with honesty and not as a proof of your abilities for the others. I am deeply thankful for the faith that he seems to have in me.

Part of the content of this thesis (half of it) is due to a collaboration with Wojciech Zakrzewski from the Department of Mathematical Science at Durham University. My gratitude for him is very big. The opportunity he gave me to not just work with him but also to go to Durham and participate in that magnificent non-perturbative environment was of a major relevance for my growth. I extend my gratitude for all the people I met there, and somehow contributed to all that. In particular, Laura da Costa and Karen Blundell in Grey College. Also, I must mention how lucky I was in meeting David Tapp, who helped me with everything I needed and was (and is) a true friend, that I really hope to see again.

During almost my entire PhD studies time I was the only student in the group which made 
my life even harder. In this last year, however, Vinicius Aurichio joined us and this was great for me. I am very glad for his companionship as my office mate. Also the presence of David Foster as a pos-doc gave a much more enthusiastic feeling to the place and definitely our daily discussions about math, physics and women gave me much more motivations to work. I learned a lot with him, and for that I am very grateful.

It is not even necessary to say that I could only get to this point thanks to Arlete, Marina, Natália and Mercedes. Although very far away their love for me made them always very close.

Harder than handle a $\mathrm{PhD}$, is to do it and take care of Lays... but what does not kill us makes us stronger, and I am deeply grateful to have met her and for her being sharing all this with me. I found in her the hidden symmetry that makes my happiness conserved. I wish also to thank her family that gave me a safe place to be every time a needed.

A very special thanks to Thiago Mosqueiro, who developed this amazing Latex template that makes my thesis looks more important than it is. 
'...when you have eliminated all which is impossible, then whatever remains, however improbable, must be the truth.'

Sherlock Holmes Quote - The Blanched Soldier 



\section{RESUMO}

LUCHINI, G. Simetrias escondidas em teorias de calibre \& quasi-integrabilidade. 2013. 113 p. Tese (Doutorado em Física Básica) - Instituto de Física de São Carlos, Universidade de São Paulo, São Carlos, 2013.

Essa tese discute algumas extensões de idéias e técnicas usadas em teorias de campos integráveis para tratar teorias que não são integráveis. Sua apresentação é feita em duas partes. A primeira tem como tema teorias de calibre em 3 e 4 dimensões; propomos o que chamamos de equação integral para uma tal teoria, o que nos permite de maneira natural a construção de suas cargas invariantes de calibre, e independentes da parametrização do espaço-tempo. A definição de cargas conservadas in variantes de calibre em teorias não-Abelianas ainda é um assunto em aberto e acreditamos que a nossa solução pode ser um primeiro passo em seu entendimento. A formulação integral mostra uma conexão profunda entre diferentes teorias de calibre: elas compartilham da mesma estrutura básica quando formuladas no espaço dos laços. Mais ainda, em nossa construção os argumentos que levam à conservação das cargas são dinâmicos e independentes de qualquer solução particular. Na segunda parte discutimos o recentemente introduzido conceito de quasi-integrabilidade: em $(1+1)$ dimensões existem modelos não integráveis que admitem soluções solitonicas com propriedades similares àquelas de teorias integráveis. Estudamos o caso de um modelo que consiste de uma deformação (não-integrável) da equação de Schrödinger não-linear (NLS), proveniente de um potencial mais geral, obtido a partir do caso integrável. O que se busca é desenvolver uma abordagem matemática sistemática para tratar teorias mais realistas (e portanto não integráveis), algo bastante relevante do ponto de vista de aplicações; o modelo NLS aparece em diversas áreas da física, especialmente no contexto de fibra ótica e condensação de Bose-Einstein. O problema foi tratado de maneira analítica e numérica, e os resultados se mostram interessantes. De fato, sendo a teoria não integrável não é encontrado um conjunto com infinitas cargas conservadas, mas, pode-se encontrar um conjunto com infinitas cargas assintoticamente conservadas, i.e., quando dois solitons colidem as cargas que eles tinham antes tem os seus valores alterados, 
mas após a colisão, os valores inicias, de antes do espalhamento, são recobrados.

PAlavRAS-ChAVE: Solitons. Formulação de curvature nula. Simetrias escondidas. Espaço de laços. Cargas conservadas. 


\section{ABSTRACT}

LUCHINI, G. Hidden symmetries in gauge theories \& quasi-integrablility. 2013. 113 p. Tese (Doutorado em Física Básica) - Instituto de Física de São Carlos, Universidade de São Paulo, São Carlos, 2013.

This thesis is about some extensions of the ideas and techniques used in integrable field theories to deal with non-integrable theories. It is presented in two parts. The first part deals with gauge theories in 3 and 4 dimensional space-time; we propose what we call the integral formulation of them, which at the end give us a natural way of defining the conserved charges that are gauge invariant and do not depend on the parametrisation of space-time. The definition of gauge invariant conserved charges in non-Abelian gauge theories is an open issue in physics and we think our solution might be a first step into its full understanding. The integral formulation shows a deeper connection between different gauge theories: they share the same basic structure when written in the loop space. Moreover, in our construction the arguments leading to the conservation of the charges are dynamical and independent of the particular solution. In the second part we discuss the recently introduced concept called quasi-integrability: one observes soliton-like configurations evolving through non-integrable equations having properties similar to those expected for integrable theories. We study the case of a model which is a deformation of the non-linear Schrödinger equation consisting of a more general potential, connected in a way with the integrable one. The idea is to develop a mathematical approach to treat more realistic theories, which is in particular very important from the point of view of applications; the NLS model appears in many branches of physics, specially in optical fibres and Bose-Einstein condensation. The problem was treated analytically and numerically, and the results are interesting. Indeed, due to the fact that the model is not integrable one does not find an infinite number of conserved charges but, instead, a set of infinitely many charges that are asymptotically conserved, i.e., when two solitons undergo a scattering process the charges they carry before the collision change, but after the collision their values are recovered. 
KEYWORDs: Solitons. Zero curvature formulation. Hidden symmetries. Loop space. Conserved charges. 


\section{LIST OF FIGURES}

1.1 A 1-soliton solution propagates through the string of pendula. The energy is not dissipated, so, after the pendulum flips 180 degrees it starts to decelerate, and stops at the bottom, without wiggling. . . . . . . . . . . . .

2.1 The $\frac{1}{1}$ factor appears due to the symmetry relating the $n$ ! integrations in the path-ordered product.

2.2 One can use a family of homotopically equivalent loops to scan a 2-dimensional surface.

2.3 The zero curvature implies that the Wilson line is independent of the path.

This leads to a conservation law.

2.4 On the left, a surface $\Sigma$ in $M$ is scanned with loops based at $x_{\mathrm{R}}$. On the right, this surface is represented in $L M$, where each loop in $M$ corresponds to a point and the surface from $x_{\mathrm{R}}$ to the boundary $\partial \Sigma$ is a path. A varliation of this surface, leaving the boundary fixed, is also represented in $L M$. . . .

2.5 The border of the surface is kept fix while performing the variation. When the surfaces are closed, the border is contracted to $x_{\mathrm{R}}$ and the initial surface $(\zeta=0)$ becomes the closed infinitesimal surface $\Sigma_{R}$ while the final surface $(\zeta=2 \pi)$ becomes the boundary of a volume. . . . . . . . . . .

3.1 The surface independence of $V$ means that it can be calculated from the infinitesimal loop around $x_{\mathrm{R}}$ (the initial point in loop space) to the boundary loop $S_{\propto}^{1}$ (the final point in loop space) using any of the two surfaces (paths in loop space) presented here.

4.1 When the volume $\Omega$ becomes the infinitesimal cube the integral equations imply the differential Yang-Mills equations. The big arrows on the bottom and top surfaces indicate the sign of $\frac{d x^{\mu}}{d \tau}$. 
5.1 Plot of $|\psi|^{2}$ against $x$ for the one-soliton solution of the unperturbed NLS model. . . . . . . . . . . . . . . . . . . . p. 87

5.2 Trajectories of two Solitons at $v=0.4(\epsilon=0) \ldots \ldots$. . . . . . . . 88

5.3 Trajectories of two solitons at rest $(\epsilon=0) \ldots \ldots$ p. 88

5.4 Heights of the solitons originaly at rest $(\epsilon=0) \ldots \ldots$ p. 89

5.5 Trajectories of two solitons at $v=0.4(\epsilon=0.06) \ldots \ldots$ p. 90

5.6 Trajectories (and the energy) of two solitons at rest $(\epsilon=0.06) \ldots$ p. 91

5.7 Trajectories (and the energy) of two solitons at rest $(\epsilon=-0.06) \ldots$ p. 92

5.8 Heights of the two solitons observed in their scattering at rest $(\epsilon=-0.06$ $c=0) \ldots \ldots \ldots \ldots \ldots \ldots$. . . . . . . . . . . . . . . . . . . . . .

5.9 Time integrated anomaly of two solitons sent at $v=0.4(\epsilon=0.06) \ldots \quad$ p. 93

5.10 Time integrated anomaly of two solitons at rest $(\epsilon=0.06) \ldots$. . . . . . p. 94

5.11 Time integrated anomaly of two solitons sent at $v=0.4(\epsilon=-0.06) \ldots \quad$ p. 94

5.12 Time integrated anomaly of two solitons at rest $(\epsilon=-0.06) \ldots$ p. 94

A.1 The regularisation of the Wilson line operator is done by replacing the path that passes through to the origin by a path going around it. . . . . . . . . p. 101 


\section{SUMMARY}

1 Introduction

p. 15

2 Hidden Symmetries, Stokes theorem and conservation laws

2.1 The standard non-Abelian Stokes theorem . . . . . . . . . . . . p. 30

2.2 Generalisation of the Stokes theorem . . . . . . . . . . . . . p. 36

3 Integral formulation of theories in $2+1$ dimensions $\quad$ p. 41

3.1 The integral equations of Chern-Simons theory . . . . . . . . . . . . p. p. 41

3.2 The integral equations of $(2+1)$-dimensional Yang-Mills theory . . . . . p. 45

4 The integral Yang-Mills equation in $3+1$ dimensions $\quad$ p. 47

4.1 The full Yang-Mills integral equation . . . . . . . . . . . . p. 47

4.2 The self-dual sector . . . . . . . . . . . . . . . . . p. 51

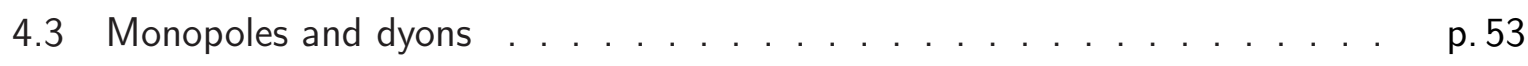

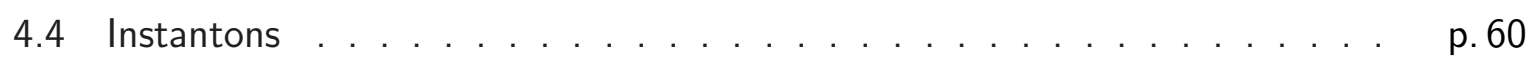

4.5 Merons . . . . . . . . . . . . . . . . . . . . . . . . . . . . p. 63

5 Quasi-integrable deformation of the non-linear Schrödinger equation $\quad$ p. 67

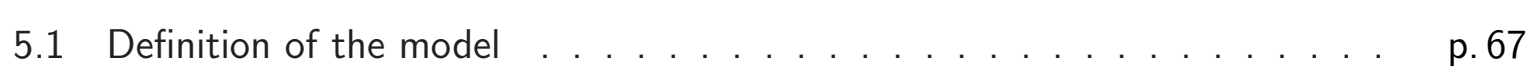

5.1 .1 On the parity symmetry . . . . . . . . . . . . . . . p. 76

5.2 Dynamics versus parity . . . . . . . . . . . . . . . . . . . . p. 78

5.2 .1 Deformations of the NLS theory . . . . . . . . . . . . . . . . . . . p.79 
5.3 The parity properties of NLS solitons . . . . . . . . . . . p. 83

5.3 .1 The one-soliton solutions ................ . . . . 83

5.3.2 The two-soliton solutions .............. . . . . . . 84

5.4 Numerical analysis . . . . . . . . . . . . . . . . . . . . . p. 87

5.4 .1 The NLS model . . . . . . . . . . . . . . . . . . . p. 87

5.4.2 The modified model with $\varepsilon \neq 0 \ldots . . \ldots$ p. 90

6 Final Comments $\quad$ p. 95

$\begin{array}{ll}\text { REFERENCES } & \text { p. } 97\end{array}$

Appendix A - Regularisation of Wilson lines p. 101

Appendix B - Explicity quantities involved in equation (5.1.25) p. 107

$\begin{array}{ll}\text { Appendix C - The Hirota solutions } & \text { p. } 111\end{array}$ 


\section{CHAPTER 1}

\section{Introduction}

All mentors have a way of seeing more of our faults than we would like.

Padmé Amidala

Great achievements in physics were done from attempts to put together apparently conflicting theories. For instance, the incompatibility between Maxwell's electromagnetism and the Galilean covariance led to the development of special relativity; the loss of energy of the orbiting electron predicted by classical electrodynamics and the stability of the atom (an empirical fact), among others, led to the construction of the laws of quantum mechanics; the difficulty in introducing Newton's gravity into the principles of special relativity gave birth to the general theory of relativity.

Our main approach to understand Nature within its different aspects, as in the solution of the problems mentioned above, is through symmetry principles. Three of the four interactions, namely the weak, strong and electromagnetic, are based on the so called gauge principle while the gravitational interaction relies on the equivalence principle.

The symmetries are not just fundamental as a basic ingredient in the construction of the theories, letting the physical degrees of freedom be identified, but are also important in the development of systematic methods leading to solutions and/or observables.

In particular, symmetries referred to as "hidden" play a major role in the understanding of non-linear field theories in 2-dimensional space-time and in the development of exact/nonperturbative methods to treat them. Such theories are certainly important in many branches of condensed matter and, in higher energy physics, are used as toy-models for realistic scenarios. The existence of soliton-like solutions in 3 and 4 dimensions (and the lack of them) leads to the quest of improvement and/or development of powerful tools such as the zero curvature representation used for integrable theories in 2 dimensions(1, 2). 


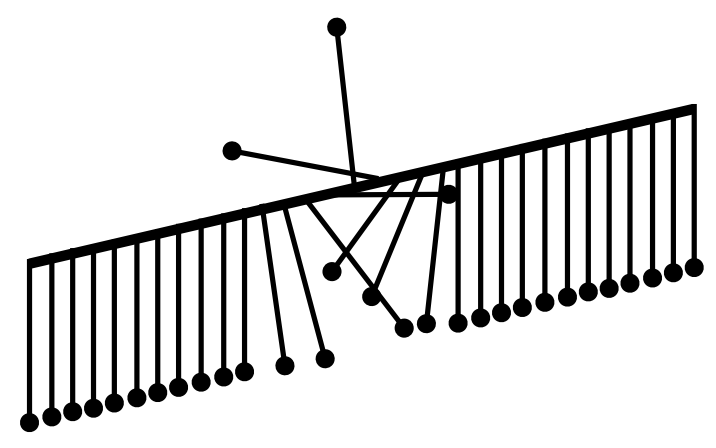

Figure 1.1 - A 1-soliton solution propagates through the string of pendula. The energy is not dissipated, so, after the pendulum flips 180 degrees it starts to decelerate, and stops at the bottom, without wiggling.

To be concrete let us consider the example of the well-known sine-Gordon equation describing the dynamics of the real scalar field $\varphi(t, x)$ :

$$
\partial_{t}^{2} \varphi-\partial_{x}^{2} \varphi+\frac{m^{2}}{\beta} \sin (\beta \varphi)=0
$$

This equation appears in diverse phenomena in physics, and in particular as the continuous version of the mechanical model presented in figure (1.1): a set of pendula attached to a rubber band. In that case $\varphi$ stands for the angle of the straight rod holding the mass blob to the rubber band, and $m$ and $\beta$ are some combinations of the value of gravity, the length of the rod, the separation between two pendula and the torsion of the rubber band. For a small angle we can consider $\sin (\beta \varphi) \approx \beta \varphi$, and (1.0.1) becomes the linear Klein-Gordon equation whose solutions are ordinary (linear) propagating waves. This is the perturbative sector of the theory. Perturbative methods are very well developed in physics and roughly speaking everything in quantum electrodynamics is done using them; the standard model, one of the cornerstones of modern science, is a consequence of the success of that approach. On the other hand this string of pendula presents very interesting configurations in the non-perturbative sector. In figure (1.1) the 1-soliton solution $\varphi(t, x)=\frac{4}{\beta} \arctan e^{\frac{m}{\sqrt{1-v^{2}}}(x-v t)}$ is sketched. In opposition to the linear waves a soliton do not admit the superposition principle. It propagates with constant velocity without changing its shape or dissipating energy, and when two of them undergo a scattering process the only effect they feel is a shift from the position they would have if they were propagating freely. These features lead to the interpretation of solitons as particles. Besides, generally the coupling of solitons is inversely proportional to the coupling constant of fundamental particles, so that they tend to be free in the strong coupling regime, and this is certainly interesting when (as often happens) there is a duality between solitons and particles(3) involving the weak and strong coupling sectors. Their stability and the behaviour 
just described arise* from the existence of infinitely many conserved quantities (often called charges) that can eventually be obtained when one recast the dynamical equations of the theory as a zero curvature equation, $G_{t x} \equiv \partial_{t} C_{x}-\partial_{x} C_{t}+\left[C_{t}, C_{x}\right]=0$, i.e., the vanishing of the curvature of the Lie algebra valued 1-form connection $C=C_{t} d t+C_{x} d x$, a functional of the fields and its derivatives, implies the equations of motion of the theory and vice versa.

In the case of sine-Gordon theory if we take the components of the connection as

$$
C_{t}=\frac{m}{4}\left(\begin{array}{cc}
\frac{i \beta}{m} \frac{\partial \varphi}{\partial x} & \left(e^{i \beta \frac{\varphi}{2}}+\frac{1}{\lambda} e^{-i \beta \frac{\varphi}{2}}\right) \\
\left(e^{i \beta \frac{\varphi}{2}}+\lambda e^{-i \beta \frac{\varphi}{2}}\right) & -\frac{i \beta}{m} \frac{\partial \varphi}{\partial x}
\end{array}\right)
$$

and

$$
C_{x}=\frac{m}{4}\left(\begin{array}{cc}
\frac{i \beta}{m} \frac{\partial \varphi}{\partial t} & \left(e^{i \beta \frac{\varphi}{2}}-\frac{1}{\lambda} e^{-i \beta \frac{\varphi}{2}}\right) \\
\left(-e^{i \beta \frac{\varphi}{2}}+\lambda e^{-i \beta \frac{\varphi}{2}}\right) & -\frac{i \beta}{m} \frac{\partial \varphi}{\partial t}
\end{array}\right),
$$

then the curvature becomes

$$
G_{t x}=\frac{i m}{4}\left(\partial_{t}^{2} \varphi-\partial_{x}^{2} \varphi+\frac{m^{2}}{\beta} \sin (\beta \varphi)\right)\left(\begin{array}{cc}
1 & 0 \\
0 & -1
\end{array}\right),
$$

and we clearly see that $G_{t x}=0 \Leftrightarrow \partial_{t}^{2} \varphi-\partial_{x}^{2} \varphi+\frac{m^{2}}{\beta} \sin (\beta \varphi)=0$.

There is no recipe to get a flat connection like this for a given theory. Its existence is related to the integrability of the theory(5). In fact, the curvature $G_{t x}$ comes from the compatibility conditiont of the associated linear problem described by the set of two equations $\left(\partial_{\mu}+C_{\mu}\right) \Psi=0$, with $\mu=0,1$ corresponding to the $t$ and $x$ components. The quantity $\Psi$ is an element of the group $G$. This equation can be solved if the connection is flat: $C_{\mu}=-\partial_{\mu} \Psi \Psi^{-1}$. A gauge transformation $C_{\mu} \rightarrow C_{\mu}^{\prime} \equiv h C_{\mu} h^{-1}-\partial_{\mu} h h^{-1}$ with $h$ in the gauge group $G$ implies that $G_{\mu \nu} \rightarrow h G_{\mu \nu} h^{-1}$, and therefore does not affect the zero curvature representation; the curvature remains zero. However, the connection changes and due to the non-homogeneity of this transformation one can produce non-trivial solutions for $\Psi$ from very simple ones which is very powerful. For instance, in the string of pendula we can then start from the vacuum configuration, where every pendula are at rest at the bottom, and with such a gauge transformation get a highly non-trivial configuration, which of course is a solution of the equation of motion since the curvature of this gauged connection is also zero.

\footnotetext{
${ }^{*}$ In the case of soliton-like solutions in $d+1$-dimensions, with $d>1$, the stability of the so called topological solitons (4) is related to their topological charges.

†The system is said compatible if $\left[\partial_{t}+C_{t}, \partial_{x}+C_{x}\right]=0$.
} 
Notice that the parameter $\lambda$ was introduced in the definition of the connection in a way that it does not appear in the equation of motion. It is called the spectral parameter and is crucial in the obtention of an infinite number of conserved charges, which, as said before, is responsible for the stability of the soliton. It must be noticed that these charges are not, a priori, related to the Noether's charges, i.e., with the symmetries of the equation of motion. Indeed, equation (1.0.1) has just the 2-dimensional Poincarè invariance and a discrete symmetry under $\varphi \rightarrow \frac{2 \pi n}{\beta}+\varphi$. This is certainly far from being a set of infinitely many symmetries generating that infinite number of conserved charges. Instead, such symmetries giving the stability of the solitons are related to the fact that the charge operator undergoes an iso-spectral evolution in time, and therefore, its eigenvalues (the charges) are conserved; that time evolution is then a symmetry, but this is only revealed when the zero curvature representation of the equations of motion of the theory is found, and that is why one refers to it as a hidden symmetry. This charge operator can be naturally obtained with the use of the non-Abelian Stokes theorem:

$$
P_{1} e^{-\oint_{\partial \Sigma} C_{\mu} d x^{\mu}}=P_{2} e^{\int_{\Sigma} W^{-1} G_{\mu \nu} W d x^{\mu}} d x^{\nu} .
$$

The I.h.s of the equation above is the path-ordered integral of the connection $C$ along a curve, which is the boundary of the 2-dimensional surface $\Sigma$. That $P_{1}$ refers to this ordering. Let us be more precise. This quantity is obtained from the following equation

$$
\frac{d W}{d \sigma}+C_{\mu} \frac{d x^{\mu}}{d \sigma} W=0
$$

which defines the Wilson line $W$ (in a finite representation of $C$, a matrix) along a curve parametrised by $\sigma$. The solution of it is given by an infinite series

$$
W(\sigma)=\mathbb{1}-\int_{0}^{\sigma} C_{\mu}\left(\sigma^{\prime}\right) \frac{d x^{\mu}}{d \sigma^{\prime}}+\int_{0}^{\sigma} C_{\mu}\left(\sigma^{\prime}\right) \frac{d x^{\mu}}{d \sigma^{\prime}} \int_{0}^{\sigma^{\prime}} C_{\nu}\left(\sigma^{\prime \prime}\right) \frac{d x^{\nu}}{d \sigma^{\prime \prime}} W\left(\sigma^{\prime \prime}\right) d \sigma^{\prime \prime} d \sigma^{\prime}
$$

up to a multiplication by a constant element from the right, and $\sigma \geq \sigma^{\prime} \geq \sigma^{\prime \prime}$, etc. This series can be formally written as an exponential. Due to the non-Abelian character of $C$, the order it appears in the products matters, and to guarantee that this is respected we introduce $P_{1}$. The r.h.s of the non-Abelian Stokes theorem is the ordered integral of the curvature of $C, G=d C+C \wedge C$, conjugated with $W$, on the surface $\Sigma$. As we discuss in chapter 2 this surface is scanned with loops based at $x_{\mathrm{R}}$, a point on its border we call reference point. Every point on $\Sigma$ belongs to a unique loop, and every loop can be obtained by smooth variations from the point-loop around $x_{\mathrm{R}}$, until we reach the border, which is the final loop. So, the Wilson line appearing inside the integral on the r.h.s of the theorem above is calculated along each such loop from the reference point. Then, we can get $W$ on the curve $\partial \Sigma$ by considering that this curve is the result of variations from the point-loop. This point of view was presented 
first in (6) and we reproduce it in chapter 2. One finds that it is possible to calculate $W$ using the equation

$$
\frac{d W}{d \tau}-W \int d \sigma W^{-1} G_{\mu \nu} W \frac{\partial x^{\mu}}{\partial \sigma} \frac{\partial x^{\nu}}{\partial \tau}=0
$$

where $\tau$ parametrises the variation from one loop to another, and the integration appearing here is performed along the entire loop. The solution of it gives exactly the r.h.s of the non-Abelian Stokes theorem, where $P_{2}$ stands for the ordering with respect to $\tau$, that we call surface-ordering.

Once a zero curvature representation for the equations of motion is found, this theorem implies that the Wilson line along any of the loops $\Gamma_{c}$ scanning the surface $\Sigma$ is the same, i.e.,

$$
W_{\Gamma_{\mathrm{c}}}=P_{1} e^{-\oint C}=1
$$

This leads to a very important property of the Wilson line: it is path independent. This is not difficult to see. Consider that the loop $\Gamma_{c}$ is made of the composition of two paths: $\Gamma_{\mathrm{c}}=\Gamma_{2} \circ \Gamma_{1}$. Then, we use the fact that the Wilson line follows such a decomposition, becoming $W_{\Gamma_{\mathrm{c}}}=W_{\Gamma_{2}} \cdot W_{\Gamma_{1}}$. Next, we take the reverse order of the path $\Gamma_{2}$, and using the fact that $W_{\Gamma_{2}^{-1}}=W_{\Gamma_{2}}^{-1}$ and that $W_{\Gamma_{\mathrm{c}}}=1$, we get $W_{\Gamma_{1}}=W_{\Gamma_{2}}$; the Wilson line calculated from $x_{\mathrm{R}}$ to the point $\Gamma_{1} \cap \Gamma_{2}$ is the same, independently of the path.

Now, the path independence is what leads to the conserved charges. We split space-time into space and time, and take each of these paths $\Gamma_{1}$ and $\Gamma_{2}$ to be composed by two other paths. The path $\Gamma_{1}$ is made of a path that goes from the reference point to the spatial boundary, at constant time, say $t=0$. This path is called $\Gamma_{0}$ to stress the fact that it takes the whole space at time zero. Then the second path forming $\Gamma_{1}$ is the "time evolution" of the spatial boundary, that goes from $t=0$ to some given $t>0$. This path is called $\Gamma_{\infty}$ to emphasize that it is just the point on the border, that we will take to be at infinity, that "goes up in time" until we reach the point $\Gamma_{1} \cap \Gamma_{2}$, at spatial infinity and time $t>0$. Now the path $\Gamma_{2}^{-1}$ starts with the "time evolution" of the reference point, from time zero to that time $t>0$. This path is the $\Gamma_{-\infty}$. Then we compose it with the path that goes from the reference point at time $t>0$ to the border of space at this time, i.e., this path, called $\Gamma_{t}$, is the one that sweeps the whole space, like $\Gamma_{0}$, but at time $t>0$. At the end we are going from $x_{\mathrm{R}}$ to the spatial border at time $t>0$ using two different paths. We use again the fact that the Wilson line follows the decomposition of the path to get $W_{\Gamma_{-\infty}}^{-1} \cdot W_{\Gamma_{t}}^{-1} \cdot W_{\Gamma_{\infty}} \cdot W_{\Gamma_{0}}=1$. With appropriate boundary conditions we can make the Wilson lines corresponding to the "time evolution" of the borders coincide (and we rename them in a very suggestive way as $U(t)$ ) 
and get the aforementioned iso-spectral evolution

$$
W_{\Gamma_{t}}=U(t) \cdot W_{\Gamma_{0}} \cdot U^{-1}(t)
$$

Then it becomes clear that the eigenvalues of $W$ calculated over the whole space at a certain time slice is the same for any time. This is a conservation law, and it appeared here because we had a flat connection $C$, so that $d C+C \wedge C=0$, which through the Stokes theorem gave the path independence of the Wilson line.

In 1998 Luiz Agostinho Ferreira, Joaquin Sanchez-Guillén and Orlando Alvarez proposed that maybe a first step into the construction of the concept of integrability for theories in a higher (or any) dimensional space-time could be encoded in a generalisation of this charge operator. If the space-time $M$ is now $d+1$-dimensional, a generalisation of $W$ would involve a connection which is a differential form of higher degree; a $d$-form. Remarkably, the demonstration they give for the non-Abelian Stokes theorem (that first appeared in (6), then in (7) and can also be found in details in (8)) gives a systematic method to generalise it for connections of higher degree and also (or consequently) to define the objects that generalise $W$. In fact, what they noticed is that the natural environment to extend the zero curvature representation and therefore the path-independence of the Wilson line, is the so called loop space. For a $d+1$-dimensional space-time $M$ the loop space $L M$ consists of the set of maps from the $(d-1)$-sphere $S^{d-1}$ to $M$, keeping the image of one point, for instance the north-pole of $S^{d-1}$, fixed as the reference point $x_{\mathrm{R}}$ in $M$. For $d=2$, for instance, the image of these maps are loops based on $x_{\mathrm{R}}$. A loop in $M$ corresponds to a point in $L M$. So, if we scan a 2-dimensional surface $\Sigma$ with loops, the initial loop will be a point in loop space and the final loop, the boundary of the surface, another point. The bulk of the surface consists basically of a set of loops that are continuously deformed from the initial one, the point-loop around $x_{\mathrm{R}}$; this corresponds to a path in loop space. Notice now that for $d=1$ the loop space is the set of mappings from the sphere $S^{0}$ to the 2-dimensional space-time. This sphere is in fact made of two points. One has its image fixed in $x_{\mathrm{R}}$ by construction, so the map is made from a point, the other one in $S^{0}$ to another point in space-time. So, the loop space coincides with space-time. That is a great motivation to understand how the loop space is the natural place to generalise the Stokes theorem and consequently the zero-curvature representation.

The above construction in loop space for the $d=2$ case, where the surface is scanned with loops, is exactly the way (1.0.3) was introduced. Indeed, this equation can be used to generalise the Wilson line, once, as presented in (7), the quantity $\int d \sigma W^{-1} G_{\mu \nu} W \frac{\partial x^{\mu}}{\partial \sigma} \frac{\partial x^{\nu}}{\partial \tau}$ is a connection in loop space. Then, the generalisation of this term is done by replacing $G$ by a general 2-form $B$, so it reads $\mathcal{A}=\int_{0}^{\sigma} d \sigma^{\prime} W^{-1}\left(\sigma^{\prime}\right) B_{\mu \nu}\left(\sigma^{\prime}\right) W\left(\sigma^{\prime}\right) \frac{\partial x^{\mu}}{\partial \sigma^{\prime}} \delta x^{\nu}$. Thus, following what we said before 
the idea is to search for this connection in loop space such that $\mathcal{F}=\delta \mathcal{A}+\mathcal{A} \wedge \mathcal{A}=0$, i.e., its curvature in loop space vanishes. This would lead to a generalisation of the above mentioned path-independence, which is the property one seeks for the construction of the conserved charges. Considerable progress was done in this direction; the vanishing of such a curvature can be seen as a guide for integrability in higher dimensions. Some very interesting well-known models were studied under this perspective, and original results appear thanks to this zero curvature formulation in loop space of these models (besides the applications presented in (7) see for instance (9)).

Regardless the success of that construction in this thesis we follow a different approach. Instead of looking for a flat connection in loop space we propose that the differential equations of motion of the theory have an integral version, which is based on the standard and/or generalised Stokes theorem, whose general form in a space-time $M$ of dimension $d+1$ is

$$
P_{d-1} e^{\int_{\partial \Omega} \mathcal{A}}=P_{d} e^{\int_{\Omega} \mathcal{F}}
$$

a relation between the quantities $\mathcal{A}$ and $\mathcal{F}$, constructed from $(d-1)$ and $d$-forms respectively. On the I.h.s we have the ordered integration of $\mathcal{A}$ on the boundary of the hyper-volume $\Omega$, a sub-manifold of $M$, and on the r.h.s the ordered integration of $\mathcal{F}$ in the bulk of $\Omega$.

The idea follows more or less what we have for electromagnetism, i.e., the differential equations known as the Maxwell's equations can be integrated and with the use of Stokes theorem one gets the laws for the fluxes of electric and magnetic fields. Indeed, here we show how such an integral formulation is done for gauge theories. The integral formulation of electromagnetism in terms of fluxes (done by Faraday with his invention of the lines of fields) precedes Maxwell's differential equations and were and are very important for the understanding of the phenomena. However, non-Abelian gauge theories did not follow the same historical route, and as far as we know, the integral version of Yang-Mills theories were never presented before, so apparently this is the first time it is done.

We promote the Stokes theorem from a mathematical identity to a physical equation, which we call the integral equation. The physical fields constitute $\mathcal{A}$ and $\mathcal{F}$ above, more or less like $C_{\mu}$ is written in terms of the physical fields in the zero curvature formulation. Then we write the integral equation in a way that when $\Omega$ is considered infinitesimal the differential equations are recovered.

The key point in this formulation is that the integral equation gives the possibility of finding the path independence property, which is the important thing in the obtention of the conserved charges, without going through the need of a zero curvature. Lets see how it happens for the 
case of theories in $(1+1)$-dimensional space-time, i.e., let us reformulate the ideas presented before from another perspective. Take $\Omega$ a 1-dimensional sub-manifold of $M$, which is a path going from $x_{\mathrm{R}}$ to $x_{\mathrm{f}}$. Take a field $g(x)$, and element of the group $G$. We then construct the quantity $g\left(x_{\mathrm{f}}\right) \cdot g^{-1}\left(x_{\mathrm{R}}\right)$, i.e., a quantity made of the field $g(x)$ on the border of $\Omega$. Then, take also the field $C_{\mu}(x)$ and define the quantity $P_{1} e^{-\int_{\Omega} C}$, on the bulk of $\Omega$. Finally we claim a relation between $g(x)$ and $C_{\mu}(x)$ given by the the integral equation

$$
g\left(x_{\mathrm{f}}\right) \cdot g^{-1}\left(x_{\mathrm{R}}\right)=P_{1} e^{-\int_{\Omega} C} .
$$

Now, if the border of $\Omega$ is fixed then the I.h.s of the above equation is fixed, for any path linking the points $x_{\mathrm{R}}$ and $x_{\mathrm{f}}$, so, as a consequence of the integral equation the quantity on the r.h.s $P_{1} e^{-\int_{\Omega} C}$, which is simply the Wilson line, obtained from (1.0.2), is path independent, which is the property we want. We achieved the path independence without having to talk about a zero curvature.

If $\Omega$ is an infinitesimal path then $g(x) \approx g\left(x_{\mathrm{R}}\right)+\partial_{\mu} g\left(x_{\mathrm{R}}\right) \delta x^{\mu}$ and the I.h.s becomes $\mathbb{1}+\partial_{\mu} g\left(x_{\mathrm{R}}\right) \cdot g^{-1}\left(x_{\mathrm{R}}\right) \delta x^{\mu}$, while the r.h.s $\mathbb{1}-C_{\mu}\left(x_{\mathrm{R}}\right) \delta x^{\mu}$, which gives the differential equation $C_{\mu}=-\partial_{\mu} g g^{-1}$. This is exactly saying that the connection is flat, which is the condition for the solution of the associated linear problem $\left(\partial_{\mu}+C_{\mu}\right) \Psi=0$. Of course, this leads to a zero curvature, but from this point of view it is just a consequence, and not something we need from the beginning. That might seems just another point of view in this case, with no further implications, but it is crucial to the construction in higher dimensions.

Remarkably our integral formulation of gauge theories enable us to define naturally what the conserved charges are. This discussion in gauge theories is not closed: there are many attempts (see for instance (10-14)) to define charges that are conserved and also invariant under gauge transformations, a fundamental property for any physical quantity.

In the standard literature the charges associated to the Yang-Mills theory are constructed as follows. From the Yang-Mills equations $D_{\nu} F^{\nu \mu}=J^{\mu}, D_{\nu} \widetilde{F}^{\nu \mu}=0$, where $J_{\mu}$ stands for the matter current, $\widetilde{F}_{\mu \nu}=\frac{1}{2} \epsilon_{\mu \nu \rho \lambda} F^{\rho \lambda}$ is the Hodge dual of the field strength, and the covariant derivative is $D_{\mu} \star=\partial_{\mu}+i e\left[A_{\mu}, \star\right]$, one defines the quantity $j^{\mu} \equiv \partial_{\nu} F^{\nu \mu}=J^{\mu}-i e\left[A_{\nu}, F^{\nu \mu}\right]$ and its Hodge dual $\widetilde{j^{\mu}} \equiv \partial_{\nu} \widetilde{F}^{\nu \mu}=-i e\left[A_{\nu}, \widetilde{F}^{\nu \mu}\right]$, which are locally conserved due to the antisymmetry of the field strength tensor. With appropriate boundary conditions the charges coming from $j^{\mu}$ and $\widetilde{j^{\mu}}$ are written as

$$
Q_{\mathrm{YM}}=\int_{S_{\infty}^{2}} d \mathbf{S} \cdot \mathbf{E} \quad \widetilde{Q}_{\mathrm{YM}}=\int_{S_{\infty}^{2}} d \mathbf{S} \cdot \mathbf{B}
$$

\footnotetext{
$\ddagger$ Basically this can be found in any good book about the subject. One example is (15).
} 
where $E_{i}=F_{0 i}$ and $B_{i}=-\frac{1}{2} \epsilon_{i j k} F_{j k}$ are the non-Abelian electric and magnetic fields. Under a gauge transformation $h \in G$ these charges become

$$
Q_{\mathrm{YM}} \rightarrow \int_{S_{\infty}^{2}} d \mathbf{S} \cdot h \mathbf{E} g^{-1} \quad \widetilde{Q}_{\mathrm{YM}} \rightarrow \int_{S_{\infty}^{2}} d \mathbf{S} \cdot h \mathbf{B} g^{-1},
$$

and the eigenvalues of them remain invariant only under gauge transformations that go to a constant $h_{\infty}$ at infinity, $Q_{\mathrm{YM}} \rightarrow h_{\infty} Q_{\mathrm{YM}} h_{\infty}^{-1}, \widetilde{Q}_{\mathrm{YM}} \rightarrow h_{\infty} \widetilde{Q}_{\mathrm{YM}} h_{\infty}^{-1}$, and not under a general gauge transformation.

We present here what we think may be the starting point to find a solution to this problem. We borrow the idea used to build the charges responsible for the stability of the solitons in integrable field theories to get the conserved gauge invariant charges in gauge theories. The integral equations are formulated for theories in $(2+1)$-dimensional space-time, namely the Chern-Simons theory and Yang-Mills, and in $(3+1)$ dimensions we describe the Yang-Mills theory and its self-dual sector. The charge operator is found in all the cases, and we give the charges explicitly for some configurations of the $(3+1)$-dimensional Yang-Mills. Our results look very promising. In some cases the integral formulation leads naturally to the quantisation condition of the charges. Moreover it puts different gauge theories in the same status, i.e., apparently the gauge theories can be written in loop space under the same structure, as an equation for fluxes. Also, this links gauge theories and integrability, although we still do not understand how to get an infinite number of conserved charges (a crucial feature in integrable theories), if any, or to use this to construct the solutions. This remains to be investigated together with some other points we present along the thesis.

It is important to emphasize that there is a quite vast literature on integral and loop space formulations of gauge theories (see for instance (16-24)). Our approach differs in many aspects of those formulations even though it shares some of the ideas and insights permeating them.

This is the content of the first part of this thesis. All the results presented here were recently published in two articles (8) and (25) by Luiz Agostinho Ferreira and me.

The second part is about a concept called quasi-integrability introduced recently (26) by Luiz A. Ferreira and Wojciech Zakrzewski. Performing simulations with soliton-like solutions evolving through non-integrable theories they observed a behaviour very similar to that expected for integrable theories (which explains the name "quasi-integrable"). They were able to associate this with the existence of a set of infinitely many quantities that are asymptotically conserved, so giving them the name of quasi-conserved charges. 
As proposed in (26) a $(1+1)$-dimensional theory is said to be quasi-integrable if even without a zero curvature representation of its dynamical equations it presents soliton like solutions that preserve their basic physical properties like mass, topological charges, etc. when they undergo a scattering process. Also, this theory must have an infinity number of what they call quasi-conservation laws: the corresponding charges are conserved when evaluated on the one-soliton solutions, and are asymptotically conserved in the scattering of these solitons. Summarising: during the scattering of the solitons their charges can vary in time, but when the solitons are well separated after the collision they regain the values they had before the scattering. The quasi-conservation law is of the form

$$
\frac{d Q^{(n)}}{d t}=\beta_{n}(t)
$$

with $n$ an integer. The asymptotically conservation is expressed as

$$
Q^{(n)}(t \rightarrow \infty)-Q^{(n)}(t \rightarrow-\infty)=\int_{-\infty}^{\infty} d t \beta_{n}=0
$$

In (26) they considered some modifications of the sin-Gordon equation following (27) whose equations of motion were written as what is now called an anomalous zero curvature representation. Basically, the curvature is not zero for any other case but when the potential of the theory is exactly the integrable one, i.e., the potential of the integrable theory is modified in a way that it can be recovered (for instance, by fixing some parameter), so, this anomalous zero curvature becomes the zero curvature when this happens. Using the techniques already known in integrable field theories the quasi-conserved charges were found and employing analytical and numerical methods the scattering of solitons was studied and it was verified that for some special solutions the charges are indeed asymptotically conserved. The key observation of (26) was based on the fact that the two-soliton solutions satisfying (1.0.5) had the property that their fields were eigenstates of a very special space-time parity transformation

$$
P: \quad(\tilde{x}, \tilde{t}) \rightarrow(-\tilde{x},-\tilde{t}) \quad \text { with } \quad \tilde{x}=x-x_{\Delta} \quad \tilde{t}=t-t_{\Delta} .
$$

where the point $\left(x_{\Delta}, t_{\Delta}\right)$ in space-time, depends upon the parameters of the solution. Since the charges are obtained from some densities, i.e., $Q^{(n)}=\int_{-\infty}^{\infty} d x j_{0}^{(n)}$, so are the functions $\beta_{n}=\int_{-\infty}^{\infty} d x \gamma_{n}$, called integrated anomaly. Therefore, the vanishing of $\int_{-\infty}^{\infty} d t \int_{-\infty}^{\infty} d x \gamma_{n}$, follows from the properties of $\gamma_{n}$ under (1.0.6). An important remark is that the solutions for which the fields are eigenstates of the parity (1.0.6) cannot be selected by choosing appropriate initial boundary conditions; the boundary conditions are set at a given initial time and the transformation (1.0.6) relates the past and the future of the solutions. In other words, boundary conditions are kinematical statements, and the fact that a field is an eigenstate under (1.0.6) 
is a dynamical statement. The physical mechanism that guarantees that such special solutions have the required parity properties is not clear yet. That is the main motivation of this thesis: it is crucial to look at other models, with different symmetries and physical content, that are also deformations of integrable models, and analyse if the quasi-integrability phenomenon also happens.

Hence in this thesis we look at the non-linear Schrödinger (NLS) model and its perturbations. The NLS is an integrable theory. It differs from the sine-Gordon in the sense that its soliton solutions are not topological and it is non-relativistic. It worth to mention that this model appears in several branches of science, from condensed matter to biology, being extremely expressive in the context of optical fibres. Hence the understanding of quasiintegrability for this model would have very important implications. The modifications of the NLS model considered here have equations of motion of the form

$$
i \partial_{t} \psi=-\partial_{x}^{2} \psi+\frac{\partial V}{\partial|\psi|^{2}} \psi
$$

where $\psi$ is a complex scalar field and $V$ is a potential dependent only on the modulus of $\psi$. The NLS equation corresponds to $V \sim|\psi|^{4}$. The analysis of such models start by writing the equations of motion (1.0.7) as an anomalous zero curvature equation of the form

$$
\partial_{t} A_{x}-\partial_{x} A_{t}+\left[A_{x}, A_{t}\right]=\mathcal{X}
$$

where the connection $A_{\mu}$ is a functional of $\psi$ and its derivatives, and takes values in the $S L(2)$ loop algebra (Kac-Moody algebra with vanishing central element), and $\mathcal{X}$ is the anomaly that vanishes when $V$ is the NLS potential.

Then we discuss how to construct the infinite set of quasi-conserved charges by employing the standard techniques of integrable field theories known as Drinfeld-Sokolov reduction (28), or abelianisation procedure $(2,29,30)$. With them we gauge transform the $A_{x}$ component of the connection into an infinite dimensional Abelian sub-algebra of the loop algebra, generated by $T_{3}^{n} \equiv \lambda^{n} T_{3}$. Even though the anomaly $\mathcal{X}$ prevents the gauge transformation to rotate the $A_{t}$ component into the same Abelian sub-algebra, the component of the transformed curvature (1.0.8) in that sub-algebra leads to a set with infinitely many quasi-conservation laws, $\partial^{\mu} j_{\mu}^{(n)}=\gamma_{n}$, or equivalently leads to (1.0.4) with $Q^{(n)}=\int_{-\infty}^{\infty} d x j_{0}^{(n)}$ and $\beta_{n}=\int_{-\infty}^{\infty} d x \gamma_{n}$.

Next a more refined technique, involving two $\mathbb{Z}_{2}$ transformations, is used to understand the conditions for the vanishing of the integrated anomalies. The first $\mathbb{Z}_{2}$ is an order two automorphism of the $S L(2)$ loop algebra and the second is the parity transformation (1.0.6). 
For the solutions for which the field $\psi$ transforms under (1.0.6) as

$$
\psi \rightarrow e^{i \alpha} \psi^{*} \quad \text { with } \alpha \text { constant }
$$

it is shown that $\int_{-\tilde{t}_{0}}^{\tilde{t}_{0}} d t \int_{-\tilde{x}_{0}}^{\tilde{x}_{0}} d x \gamma_{n}=0$, where $\tilde{t}_{0}$ and $\tilde{x}_{0}$ are any given fixed values of the space-time coordinates $\tilde{t}$ and $\tilde{x}$, respectively, introduced in (1.0.6). This leads to

$$
Q^{(n)}\left(t=\tilde{t}_{0}+t_{\Delta}\right)=Q^{(n)}\left(t=-\tilde{t}_{0}+t_{\Delta}\right)
$$

which is a type of a mirror symmetry for the charges. Therefore, for a two-soliton solution satisfying (1.0.9), the asymptotic conservation of the charges (1.0.5) follows from this stronger conclusion.

Such results certainly unravel important structures responsible for the phenomena called quasi-integrability. They involve an anomalous zero curvature equation, internal and external $\mathbb{Z}_{2}$ symmetries, and algebraic techniques borrowed from integrable field theories. However, they rely on the assumption (1.0.9) which is a dynamical statement since it relates the past and the future of the solutions. In order to shed more light on this issue the relation between (1.0.9) and the dynamics defined by (1.0.7) is studied.

It is easier to work with the modulus and phase of $\psi$, and so the fields are parametrised as $\psi=\sqrt{R} e^{i \frac{\varphi}{2}}$, with $R$ and $\varphi$ being real scalars fields. They are separated into their eigencomponents under the parity (1.0.6), as $R=R^{(+)}+R^{(-)}$, and $\varphi=\varphi^{(+)}+\varphi^{(-)}$. The assumption (1.0.9) implies that the solution should contain only the components $\left(R^{(+)}, \varphi^{(-)}\right)$, and nothing of the pair $\left(R^{(-)}, \varphi^{(+)}\right)$. By splitting the equations of motion (1.0.7) into their even and odd components under (1.0.6), we show that there cannot exist non-trivial solutions carrying only the pair $\left(R^{(-)}, \varphi^{(+)}\right)$. In addition, if the potential $V$ in (1.0.7) is a deformation of the NLS potential, in the sense that we can expand it as

$$
V=V_{\mathrm{NLS}}+\varepsilon V_{1}+\varepsilon^{2} V_{2}+\ldots
$$

with $\varepsilon$ being a deformation parameter, then we can make even stronger statements. In such a case we expand the equations of motion and the solutions into power series in $\varepsilon$, as

$$
R^{( \pm)}=R_{0}^{( \pm)}+\varepsilon R_{1}^{( \pm)}+\varepsilon^{2} R_{2}^{( \pm)}+\ldots ; \quad \varphi^{( \pm)}=\varphi_{0}^{( \pm)}+\varepsilon \varphi_{1}^{( \pm)}+\varepsilon^{2} \varphi_{2}^{( \pm)}+\ldots
$$

If we select a zero order solution, i.e., a solution of the NLS equation, satisfying (1.0.9), carrying only the pair $\left(R_{0}^{(+)}, \varphi_{0}^{(-)}\right)$, then the equations for the first order fields, which are obviously linear in them, are such that the pair $\left(R_{1}^{(+)}, \varphi_{1}^{(-)}\right)$satisfies inhomogeneous equations, while 
the pair $\left(R_{1}^{(-)}, \varphi_{1}^{(+)}\right)$, satisfies homogeneous ones. Therefore, $\left(R_{1}^{(-)}, \varphi_{1}^{(+)}\right)=(0$, const. $)$, is a solution of the equations of motion, but $\left(R_{1}^{(+)}, \varphi_{1}^{(-)}\right)=(0$, const. $)$, is not. By selecting the first order solution such that the pair $\left(R_{1}^{(-)}, \varphi_{1}^{(+)}\right)$is absent, we see that the same happens in second order, i.e., that the pair $\left(R_{2}^{(+)}, \varphi_{2}^{(-)}\right)$also satisfies inhomogeneous equations, and the pair $\left(R_{2}^{(-)}, \varphi_{2}^{(+)}\right)$the homogeneous ones. By repeating this procedure, order by order, one can build a perturbative solution which satisfies (1.0.9), and so has charges satisfying (1.0.10). Note that the converse could not be done, i.e., we cannot construct a solution involving only the pair $\left(R^{(-)}, \varphi^{(+)}\right)$. So, the dynamics dictated by (1.0.7) favours solutions of the type (1.0.9).

Finally we discuss the conditions for the soliton solutions of the NLS equation to satisfy the parity property (1.0.9). As it is well known there are two basic types of NLS soliton solutions: the bright solitons for $\eta<0$, and dark solitons for $\eta>0$, where $\eta$ is the coupling constant of the NLS potential given by $V_{N L S}=\eta|\psi|^{4}$. The names originate from the fact that the values of $|\psi|^{2}$ increase (decrease) as one approaches the core of a bright (dark) soliton. For a more detailed discussion about NLS bright/dark solitons see (31-34) and references therein. We shall show that the one-bright-soliton and the one-dark-soliton solutions of the NLS equation satisfy the condition (1.0.9), and that not all two-bright-soliton solutions satisfy it. However, one can choose the parameters of the general solution so that the corresponding two-bright-soliton solutions do satisfy (1.0.9). This involves a choice of the relative phase between the two one-bright-solitons forming the two-soliton solution. Therefore, our perturbative expansion explained above can be used to build a sub-sector of two-brightsoliton solutions of (1.0.7) that obeys $(1.0 .9)$ and so has charges satisfying (1.0.10). This would constitute our quasi-integrable sub-model of (1.0.7). We do not analyse in this thesis the two-dark soliton solutions of the NLS equation basically for conciseness. The construction of the general two-dark soliton solution requires a modification of the Hirota's method described in appendix $\mathrm{C}$ for the case of bright solitons. In addition, our numerical code would have to be altered to deal with dark solitons.

Despite the fact that the equations of motion satisfied by the $n$-order fields $\left(R_{n}^{( \pm)}, \varphi_{n}^{( \pm)}\right)$ are linear, the coefficients are highly non-linear in the lower order fields and so, unfortunately, these equations are not easy to solve. We then use numerical methods to study the properties of our solutions. In addition, such numerical analysis can clarify possible convergence issues of our perturbative expansions. We chose to perform our numerical simulations for a potential of the form

$$
V=\frac{2 \eta}{2+\varepsilon}\left(|\psi|^{2}\right)^{2+\varepsilon} \quad \eta<0
$$


The choice of such potential is rather arbitrary. It possesses a property however which might be relevant, i.e. it does not shift the vacuum of the NLS potential. Other choices like those shown in (5.2.4) may introduce additional vacua besides $\psi=0$.

With the huge contribution of Wojciech Zakrzewski from Durham University several simulations were done using the 4th order Runge Kutta method of simulating the time evolution. These simulations involved the NLS case with the two bright solitons sent towards each other with different values of velocity (including $v=0$ ) and for various values of the relative phase. We then repeated that for the modified models. We looked at various values of $\varepsilon$ and have found that the numerical results were reliable for only a small range of $\varepsilon$ around 0 . For very small values we saw no difference from the results for the NLS model but for $|\varepsilon| \sim 0.1$ or $\sim 0.2$ the results of the simulations became less reliable. Hence, we are quite confident of our results for $|\varepsilon|<0.1$ and in the numerical section we present the results for $\varepsilon= \pm 0.06$. Also the results for the anomaly as seen in the simulations are presented and they confirm our expectations.

The results about the quasi-integrable deformations of the NLS theory presented here were published by Luiz Ferreira, Wojciech Zakrzewski and me in (35). 


\section{CHAPTER 2}

\section{Hidden Symmetries, Stokes theorem and conservation laws}

Non-linear field theories are ubiquitous in Nature. Such theories, in opposition to the linear ones (e.g. electromagnetism) do not admit the superposition principle and in general the solutions are not only harder to be found but also they behave differently when compared to linear waves. In particular the soliton solutions(? ) propagate with constant velocity without changing their shape or dissipating energy, and when two of them undergo a scattering process the only effect they feel is a shift from the position they would have if there was no scattering. These features lead to the interpretation of solitons as particles. Their stability and the behaviour just described arise from the existence of infinitely many conserved quantities that can eventually be obtained when one recast the dynamical equations of the theory as a zero curvature equation(1), $G_{t x} \equiv \partial_{t} C_{x}-\partial_{x} C_{t}+\left[C_{t}, C_{x}\right]=0$, i.e., the vanishing of the curvature of the Lie algebra valued 1 -form connection $C=C_{t} d t+C_{x} d x$, a functional of the fields and its derivatives, implies the equations of motion of the theory and vice versa. There is no recipe to build such a connection, and also the set of theories that can be described in this way (known as integrable) is not very big.

One can immediately notice that the gauge invariance of the zero curvature equation reveals a new (hidden) symmetry of the theory. We now want to discuss how to build up the conserved charges. For theories in 2-dimensions this is done through the (standard*) nonAbelian Stokes theorem, and in the next section we shall prove it following the approach in (6).

\footnotetext{
*The label "standard" here is because we intend to generalise this theorem later, remaining at the end with the generalised and the standard non-Abelian Stokes theorems.
} 


\subsection{The standard non-Abelian Stokes theorem}

Let $M$ be the 2-dimensional space-time manifold. We introduce the Wilson line $W$, a non-local object defined by the integration of the first order linear equation

$$
\frac{d W}{d \sigma}+C_{\mu} \frac{d x^{\mu}}{d \sigma} W=0
$$

along a curve $\Gamma$, a 1-dimensional sub-manifold of $M$. The points of $\Gamma$ are parametrised by $\sigma \in[0,2 \pi]$, so, $x^{\mu}=x^{\mu}(\sigma)$; the initial point (here called the reference point) is $x^{\mu}(0) \equiv x_{\mathrm{R}}$, and the final point, $x^{\mu}(2 \pi) \equiv x_{\mathrm{f}}$. Together with the equation we set the constant element $W_{\mathrm{R}}$ being the initial condition, i.e., the value of $W$ at the reference point. This equation is solved iteratively: one integrates it from the reference point to some arbitrary point $x^{\mu}(\sigma)$ in $\Gamma$

$$
W_{\Gamma}[\sigma, 0]=W_{\mathrm{R}}-\int_{0}^{\sigma} C_{\mu}\left(\sigma^{\prime}\right) \frac{d x^{\mu}}{d \sigma^{\prime}} W_{\Gamma}\left[\sigma^{\prime}, 0\right] d \sigma^{\prime}
$$

and use this result, but for $W_{\Gamma}\left[\sigma^{\prime}, 0\right]$, inside the Wilson line in the integrand above, which gives

$$
W_{\Gamma}[\sigma, 0]=W_{\mathrm{R}}-\int_{0}^{\sigma} C_{\mu}\left(\sigma^{\prime}\right) \frac{d x^{\mu}}{d \sigma^{\prime}} W_{\mathrm{R}}+\int_{0}^{\sigma} C_{\mu}\left(\sigma^{\prime}\right) \frac{d x^{\mu}}{d \sigma^{\prime}} \int_{0}^{\sigma^{\prime}} C_{\nu}\left(\sigma^{\prime \prime}\right) \frac{d x^{\nu}}{d \sigma^{\prime \prime}} W_{\Gamma}\left[\sigma^{\prime \prime}, 0\right] d \sigma^{\prime \prime} d \sigma^{\prime}
$$

and so on, indefinitely. Notice that the product of connections appears in a certain order: the rightmost term in $C_{\mu}\left(\sigma^{\prime}\right) C_{\nu}\left(\sigma^{\prime \prime}\right) \ldots C_{\rho}\left(\sigma^{\prime \ldots \prime}\right)$ is the first one, from the reference point to the final point (the direction the path is oriented) since $\sigma \geq \sigma^{\prime} \geq \sigma^{\prime \prime} \geq \cdots \geq 0$. It is then useful to introduce the path-ordered product as

$$
\begin{aligned}
& P_{1} \int_{0}^{\sigma} \int_{0}^{\sigma^{\prime}} C_{\mu}\left(\sigma^{\prime}\right) C_{\nu}\left(\sigma^{\prime \prime}\right) \frac{d x^{\mu}}{d \sigma^{\prime}} \frac{d x^{\nu}}{d \sigma^{\prime \prime}} d \sigma^{\prime \prime} d \sigma^{\prime}= \\
& \iint_{\sigma \geq \sigma^{\prime} \geq \sigma^{\prime \prime} \geq 0} C_{\mu}\left(\sigma^{\prime}\right) C_{\nu}\left(\sigma^{\prime \prime}\right) \frac{d x^{\mu}}{d \sigma^{\prime}} \frac{d x^{\nu}}{d \sigma^{\prime \prime}} d \sigma^{\prime \prime} d \sigma^{\prime}+\iint_{\sigma \geq \sigma^{\prime \prime} \geq \sigma^{\prime} \geq 0} C_{\nu}\left(\sigma^{\prime \prime}\right) C_{\mu}\left(\sigma^{\prime}\right) \frac{d x^{\nu}}{d \sigma^{\prime \prime}} \frac{d x^{\mu}}{d \sigma^{\prime}} d \sigma^{\prime} d \sigma^{\prime \prime}
\end{aligned}
$$

to guarantee that the rightmost term always comes first. Considering a 2-dimensional plane with vertical axis $\sigma^{\prime}$ and horizontal axis $\sigma^{\prime \prime}$, the first term on the r.h.s above corresponds to the integration on the top triangle in figure (2.1), while the second term, on the bottom triangle. Due to the evident symmetry $\sigma^{\prime} \leftrightarrow \sigma^{\prime \prime}$ between these terms the result of the integrations must

†Taking the connection in a finite representation of the Lie algebra, it becomes a matrix, and $W$ is a matrix as well. 


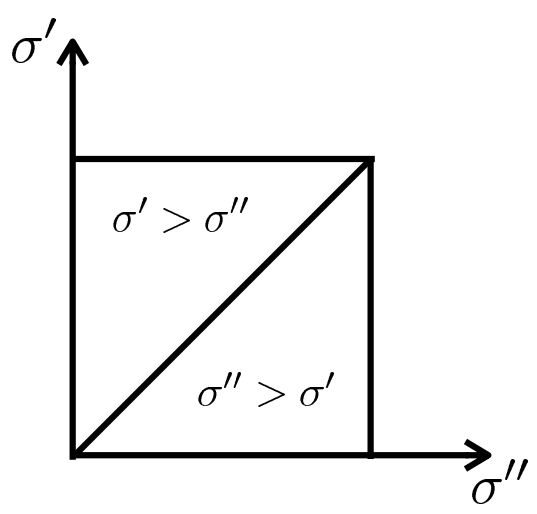

Figure 2.1 - The $\frac{1}{n !}$ factor appears due to the symmetry relating the $n$ ! integrations in the pathordered product.

be the same and therefore

$$
\begin{aligned}
\int_{0}^{\sigma} \int_{0 \quad \sigma \geq \sigma^{\prime} \geq \sigma^{\prime \prime} \geq 0}^{\sigma^{\prime}} C_{\mu}\left(\sigma^{\prime}\right) C_{\nu}\left(\sigma^{\prime \prime}\right) \frac{d x^{\mu}}{d \sigma^{\prime}} \frac{d x^{\nu}}{d \sigma^{\prime \prime}} d \sigma^{\prime \prime} d \sigma^{\prime} & =\frac{1}{2} P_{1} \int_{0}^{\sigma} \int_{0}^{\sigma^{\prime}} C_{\mu}\left(\sigma^{\prime}\right) C_{\nu}\left(\sigma^{\prime \prime}\right) \frac{d x^{\mu}}{d \sigma^{\prime}} \frac{d x^{\nu}}{d \sigma^{\prime \prime}} d \sigma^{\prime \prime} d \sigma^{\prime} \\
& \equiv \frac{1}{2} P_{1}\left(\int_{0}^{\sigma} C_{\mu}\left(\sigma^{\prime}\right) \frac{d x^{\mu}}{d \sigma^{\prime}} d \sigma^{\prime}\right)^{2} .
\end{aligned}
$$

The generalisation of the path-ordering for products involving more terms is straightforward: $P_{1}\left(C_{\mu}\left(\sigma_{1}\right) C_{\nu}\left(\sigma_{2}\right) \ldots C_{\rho}\left(\sigma_{n}\right)\right)=C_{\mu}\left(\sigma_{\pi(1)}\right) C_{\nu}\left(\sigma_{\pi(2)}\right) \ldots C_{\rho}\left(\sigma_{\pi(n)}\right)$ where $\pi$ stands for the permutation such that $\left(\sigma_{\pi(1)}\right) \geq \cdots \geq\left(\sigma_{\pi(n)}\right)$. The integrations appearing in this series are defined on simplexes. A $n$-simplex is, roughly speaking, the generalisation of triangles: they are geometrical objects with flat sides that form the convex set of their $n+1$ vertices. The very first term of the series of $W$ (after the integration constant) is the integration on a 1-simplex, which is a line. The second term, on a triangle, which is a 2-simplex. The third, on a tetrahedron, and so on. The symmetry pattern appearing in the case discussed above of the 2-simplex persists and for a $n$-dimensional cube one has $n$ ! simplexes, so, a factor $\frac{1}{n !}$ will appear in front of the path-ordered integral for the $n^{\text {th }}$ term: $\frac{1}{n !} P_{1}\left(\int_{0}^{\sigma} C_{\mu} d x^{\mu}\right)^{n}$. Finally the iterative solution of (2.1.1) can be recognised as an infinite series

$$
\begin{aligned}
W_{\Gamma}[\sigma, 0] & =\left(\mathbb{1}-P_{1} \int_{0}^{\sigma} C_{\mu} d x^{\mu}+\frac{1}{2 !} P_{1}\left(\int_{0}^{\sigma} C_{\mu} d x^{\mu}\right)^{2}-\frac{1}{3 !} P_{1}\left(\int_{0}^{\sigma} C_{\mu} d x^{\mu}\right)^{3}+\ldots\right) \cdot W_{\mathrm{R}} \\
& =\sum_{n=0}^{\infty} \frac{(-1)^{n}}{n !} P_{1}\left(\int_{0}^{\sigma} C_{\mu} d x^{\mu}\right)^{n} \cdot W_{\mathrm{R}}
\end{aligned}
$$

which can be formally written as the path-ordered exponential

$$
W_{\Gamma}[\sigma, 0]=P_{1} \exp \left(-\int_{0}^{\sigma} C_{\mu} \frac{d x^{\mu}}{d \sigma^{\prime}} d \sigma^{\prime}\right) \cdot W_{\mathrm{R}} .
$$


Although maybe obvious it is important to point out that $M$ must be arc-connected, so that we can define the path $\Gamma$ linking two points. Now, this path may be formed by the composition of several paths, for instance $\Gamma$ may be the union of a path $\Gamma_{1}$ with another, $\Gamma_{2}$, with an intersection point $\Gamma_{1} \cap \Gamma_{2}$. We denote this by $\Gamma=\Gamma_{2} \circ \Gamma_{1}$, following the path-ordering idea that the rightmost part comes first. It is not difficult to see that under such a decomposition the Wilson line is also decomposed as $W_{\Gamma}=W_{\Gamma_{2}} \cdot W_{\Gamma_{1}}$, the product being the group product once $W$ belongs to the group $G$ if $C$ is in its Lie algebra.

Consider a gauge transformation of the connection $C_{\mu} \rightarrow C_{\mu}^{\prime} \equiv h C_{\mu} h^{-1}-\partial_{\mu} h h^{-1}$ with $h$ in the gauge group $G$. We suppose that the Wilson line is transformed to $W^{\prime}$, such that the equation $\frac{d W^{\prime}}{d \sigma}+C_{\mu}^{\prime} \frac{d x^{\mu}}{d \sigma} W^{\prime}=0$ holds. Multiplying the first term of this equation by $h \cdot h^{-1}$ and with some simple manipulations, putting an $h$ term in evidence on the left, we end up with $\frac{d}{d \sigma}\left(h^{-1} \cdot W^{\prime}\right)+C_{\mu} \frac{d x^{\mu}}{d \sigma}\left(h^{-1} \cdot W^{\prime}\right)=0$, which clearly requires $W^{\prime}=h \cdot W$. However, this equation (and equation (2.1.1), more generally) defines the Wilson line up to a constant term on the right, i.e., if $h \cdot W$ is a solution of this equation, then $h \cdot W \cdot k$ is also a solution, with $k$ a constant element of the group. Let us take then $W^{\prime}=h \cdot W \cdot k$. Suppose $\Gamma=\Gamma_{2} \circ \Gamma_{1}$, and denote the intersection point $\Gamma_{1} \cap \Gamma_{2}$ by $\tilde{x}$. Then $W_{\Gamma}$ will be decomposed as discussed above and the gauge transformation will change the first part as $W_{\Gamma_{1}} \rightarrow h(\tilde{x}) \cdot W_{\Gamma_{1}} \cdot k\left(x_{\mathrm{R}}\right)$, while the second part as $W_{\Gamma_{2}} \rightarrow h\left(x_{\mathrm{f}}\right) \cdot W_{\Gamma_{2}} \cdot k(\tilde{x})$, where we used a different constant element for $\Gamma_{2}$. The constant elements can be calculated anywhere (since they are constant), and we set this to be done in the initial point of each curve. Then we have $W^{\prime}=h\left(x_{\mathrm{f}}\right) \cdot W_{\Gamma_{2}} \cdot k(\tilde{x}) \cdot h(\tilde{x}) \cdot W_{\Gamma_{1}} \cdot k\left(x_{\mathrm{R}}\right)$, which by consistency must be equal to $h\left(x_{\mathrm{f}}\right) \cdot W \cdot k\left(x_{\mathrm{R}}\right)$, so $k(\tilde{x})=h^{-1}(\tilde{x})$, and since the intersection point is arbitrary, it holds for the entire curve. Finally, we conclude that the Wilson line calculated on a given curve $\Gamma$ with boundary $\partial \Gamma=\left\{x_{\mathrm{R}} \cup x_{\mathrm{f}}\right\}$ transforms under a gauge transformation of the connection as

$$
W_{\Gamma} \rightarrow h\left(x_{\mathrm{f}}\right) \cdot W_{\Gamma} \cdot h^{-1}\left(x_{\mathrm{R}}\right)
$$

The Wilson line is defined along a curve linking two points. An important question to be understood concerns the dependence of $W$ on the curve, i.e., will the result of the integration of (2.1.1) from $x_{\mathrm{R}}$ to $x_{\mathrm{f}}$ depend on the chosen path? In order to answer that we analyse the behaviour of $W$ under variations of the type $x^{\mu} \rightarrow x^{\mu}+\delta x^{\mu}$. The simplest case is when we change the speed of the curve by changing $\sigma \rightarrow \sigma^{\prime}(\sigma)$. This produces an overall factor $\frac{d \sigma^{\prime}}{d \sigma}$ in (2.1.1), which does not change anything. So, we must consider variations that are not tangent to the curve. The reference point will remain fixed, and on the next point of the curve, infinitesimally close, we define a vector $T^{\mu}$, orthogonal to $S^{\mu} \equiv \frac{d x^{\mu}}{d \sigma}$, the tangent

$\ddagger$ In (2.1.2) we have the exponential map of a Lie algebra element. 
vector. That point will suffer a variation in the direction of this normal vector. To keep track of that a new parameter $\tau \in[0,2 \pi]$ is introduced. For $\tau=0$ the point is on the curve $\Gamma$, and for any other value it is some place else. So, the normal vector can be written as the velocity $T^{\mu} \equiv \frac{d x^{\mu}}{d \tau}$. We want the next point, infinitesimally close to this first one in $\Gamma$ to do the same, and so on. It is possible to vary every point of the curve in the same way because the vector $T^{\mu}$ is parallel transported along $\Gamma$, as one can check by calculating its Lie derivative: $£_{S} T^{\mu}=S^{\nu} \partial_{\nu} T^{\mu}-T^{\nu} \partial_{\nu} S^{\mu}=0$. For that reason once a variation (i.e., a direction of the normal vector, etc.) is defined the whole curve changes smoothly to another curve $\Gamma+\delta \Gamma$, and this process continues until $\tau=2 \pi$ defining a 2-dimensional surface.

In order to calculate the variation of the Wilson line when the curve is changed we take the variation of equation (2.1.1). Multiplying it by $W^{-1}$ from the left, after simple manipulations using that $\frac{d W}{d \sigma}=-C_{\mu} \frac{d x^{\mu}}{d \sigma} W$, and $\frac{d W^{-1}}{d \sigma}=W^{-1} C_{\mu} \frac{d x^{\mu}}{d \sigma}$, one gets $\frac{d}{d \sigma}\left(W^{-1} \delta W\right)+$ $W^{-1} \delta\left(C_{\mu} \frac{d x^{\mu}}{d \sigma}\right) W=0$, which can be integrated: $\delta W=-W \int_{0}^{\sigma} d \sigma^{\prime} W^{-1} \delta\left(C_{\mu} \frac{d x^{\mu}}{d \sigma^{\prime}}\right) W$. The integral on the r.h.s becomes $\int_{0}^{\sigma} d \sigma^{\prime} W^{-1} \delta C_{\mu} W \frac{\partial x^{\mu}}{\partial \sigma^{\prime}}+\int_{0}^{\sigma} d \sigma^{\prime} W^{-1} C_{\mu} \frac{d \delta x^{\mu}}{d \sigma^{\prime}} W$ and we use $\delta C_{\mu}=\partial_{\nu} C_{\mu} \delta x^{\nu}$ in the first term while the last one is integrated by parts, where we again make use of the equations for $W$ and $W^{-1}$, and also that $\frac{d C_{\mu}}{d \sigma}=\partial_{\nu} C_{\mu} \frac{\partial x^{\nu}}{\partial \sigma}$. After all appropriate substitutions the result is

$$
\delta W=-C_{\mu} \delta x^{\mu} W+W \int_{0}^{\sigma} d \sigma^{\prime} W^{-1} G_{\mu \nu} W \frac{\partial x^{\mu}}{\partial \sigma^{\prime}} \delta x^{\nu}
$$

where $G_{\mu \nu}=\partial_{\mu} C_{\nu}-\partial_{\nu} C_{\mu}+\left[C_{\mu}, C_{\nu}\right]$. We are omitting a more precise notation for the sake of clearness, so, lets remark that in the above equation all the Wilson lines outside the integral are defined along the curve $\Gamma$, from $\sigma=0$ to $\sigma \neq 0$. The integral defines something which is on that curve as well, so the Wilson lines appearing in the integrand, and the curvature components $G_{\mu \nu}$, are defined inside this curve at some point parametrised by $\sigma^{\prime} \in[0, \sigma]$. So, these Wilson lines are calculated using equation (2.1.1) from the reference point to $x^{\mu}\left(\sigma^{\prime}\right)$.

If both the reference point and the final point remain fixed then the first term on the r.h.s above vanishes. Moreover, since the variation is orthogonal to the curve, $\delta x^{\mu}=x^{\mu}(\tau+\delta \tau)-$ $x^{\mu}(\tau)=\frac{\partial x^{\mu}}{\partial \tau}$ and $\delta W=W(\tau+\delta \tau)-W(\tau)=\frac{d W}{d \tau} \delta \tau$, so

$$
\frac{d W}{d \tau}-W \int_{0}^{\sigma} d \sigma^{\prime} W^{-1} G_{\mu \nu} W \frac{\partial x^{\mu}}{\partial \sigma^{\prime}} \frac{\partial x^{\nu}}{\partial \tau}=0
$$

defines how the Wilson line changes in $\tau$, i.e., when we vary the curve. In fact, it shows more. This equation shows that there are two different, but equivalent ways to get $W$ along a curve. Consider $\Gamma$ to be the curve at $\tau=2 \pi$, resulting from the continuous deformation of the curve in $\tau=0$. We can get $W$ on $\Gamma$ by integration of (2.1.1) directly, knowing $W_{\mathrm{R}}$, but also, we 

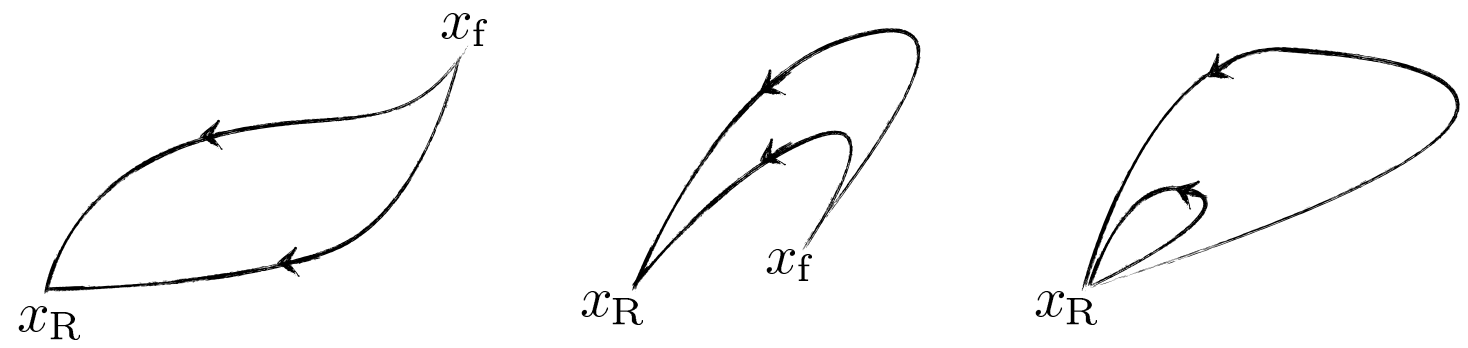

Figure 2.2 - One can use a family of homotopically equivalent loops to scan a 2-dimensional surface.

can get $W$ on $\Gamma$ by integrating (2.1.4), knowing $W$ at $\tau=0$. In particular let us consider the case where $x_{\mathrm{R}}=x_{\mathrm{f}}$. The curve $\tau=0$ becomes the infinitesimal loop around the reference point (see figure (2.2)), so that the Wilson line there coincides with $W_{\mathrm{R}}$. When this curve is varied we produce a new loop, and this process goes on until the loop labelled by $\tau=2 \pi$ is reached. This final loop encloses an area $\Sigma$, so we might refer to it as the boundary $\partial \Sigma$. Because of the nature of the variations performed, as discusses previously, each point inside $\Sigma$ belongs to a single loop, i.e., the loops do not intersect. We figure out that given a reference point in the boundary of a (simply-connected) 2-dimensional surface, we can scan this surface using a homotopic family of loops, based at this reference point. The Wilson line calculated from (2.1.1) reads, in this case,

$$
W_{\partial \Sigma}=P_{1} \exp \left(\int_{0}^{2 \pi} d \sigma C_{\mu} \frac{d x^{\mu}}{d \sigma}\right) \cdot W_{\mathrm{R}}
$$

The integration of (2.1.4) can be done similarly to that of (2.1.1). For convenience we define $\mathcal{C} \equiv \int_{0}^{2 \pi} d \sigma W^{-1} G_{\mu \nu} W \frac{\partial x^{\mu}}{\partial \sigma} \frac{\partial x^{\nu}}{\partial \tau}$, so that (2.1.4) can be written as

$$
\frac{d W}{d \tau}-W \mathcal{C}=0
$$

Integrating it iteratively one gets $W(\tau)=W_{\mathrm{R}}+W_{\mathrm{R}} \int_{0}^{\tau} \mathcal{C}\left(\tau^{\prime}\right) d \tau^{\prime}+\int_{0}^{\tau} \int_{0}^{\tau^{\prime}} W\left(\tau^{\prime \prime}\right) \mathcal{C}\left(\tau^{\prime \prime}\right) \mathcal{C}\left(\tau^{\prime}\right) d \tau^{\prime \prime} d \tau^{\prime}$, etc. and we notice the need to keep track of the ordering in the products inside the integrand. However, this time the rightmost terms are the ones that appear later in the scanning of the surface, since $\tau \geq \tau^{\prime} \geq \tau^{\prime \prime} \geq \cdots \geq 0$. Then, the solution can be formally written as

$$
W_{\partial \Sigma}=W_{\mathrm{R}} \cdot P_{2} \exp \left(\int_{0}^{2 \pi} \mathcal{C} d \tau\right)=W_{\mathrm{R}} \cdot P_{2} \exp \left(\int_{0}^{2 \pi} \int_{0}^{2 \pi} d \tau d \sigma W^{-1} G_{\mu \nu} W \frac{\partial x^{\mu}}{\partial \sigma} \frac{\partial x^{\nu}}{\partial \tau}\right)
$$

where $P_{2}$ stands for the ordering in $\tau$, referred to as surface-ordering for obvious reasons. 


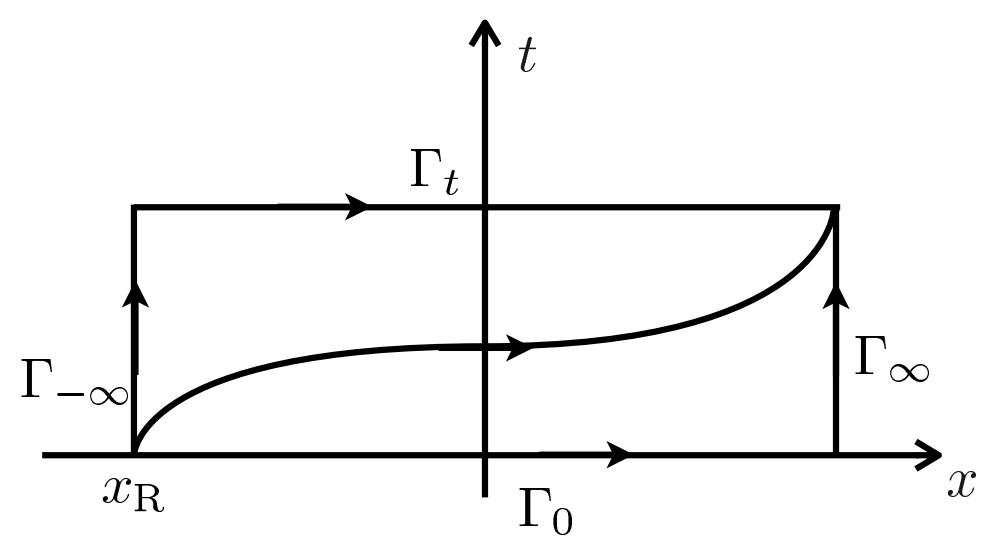

Figure 2.3 - The zero curvature implies that the Wilson line is independent of the path. This leads to a conservation law.

Since the results obtained in (2.1.5) and (2.1.7) must be the same, we have the identity

$$
P_{1} \exp \left(-\oint_{\partial \Sigma} C_{\mu} d x^{\mu}\right) \cdot W_{\mathrm{R}}=W_{\mathrm{R}} \cdot P_{2} \exp \left(\int_{\Sigma} W^{-1} G_{\mu \nu} W d x^{\mu} d x^{\nu}\right)
$$

which is the non-Abelian Stokes theorem.

Now, if we have a zero curvature representation of the equations of motion, we have that under a path deformation, keeping the border fixed, $\delta W=0$, i.e., the Wilson line is independent of the path. In particular, for a closed curve $\Gamma_{c}$, the above relation holds, and plugging $G_{\mu \nu}=0$ in the r.h.s, we get $W_{\partial \Sigma}=W_{\mathrm{R}}$, or, multiplying by $W_{\mathrm{R}}^{-1}$ from the right,

$$
P_{1} \exp \left(-\oint_{\partial \Sigma} C_{\mu} d x^{\mu}\right)=1
$$

In order to construct the conserved charges we start by splitting space-time into space and time. We consider that $M$ is flat, with a Minkowski metric. In curved space-time this separation is not trivial to be done. Then we take the closed path as $\Gamma_{c}=\Gamma_{2}^{-1} \circ \Gamma_{1}$, where $\Gamma_{1}=\Gamma_{\infty} \circ \Gamma_{0}$, linking the reference point to a final one, and $\Gamma_{2}=\Gamma_{t} \circ \Gamma_{-\infty}$, a different way to link the same two points, according to the figure (2.3). For simplicity we could consider $W_{\mathrm{R}}=1$, or in the centre of the group $Z(G)$, so that it may be dropped from (2.1.8). Or, we could just take $W_{\Gamma}=Q_{\Gamma} \cdot W_{\mathrm{R}}$. Equation (2.1.9) implies that the operator $Q_{\Gamma}$ (or equivalently the Wilson line) is independent of the path linking the reference point to the final point: $Q_{\Gamma_{\mathrm{c}}}=Q_{\Gamma_{2}}^{-1} \cdot Q_{\Gamma_{1}}=1$. Then, $Q_{\Gamma_{\mathrm{c}}}=Q_{\Gamma_{-\infty}}^{-1} \cdot Q_{\Gamma_{t}}^{-1} \cdot Q_{\Gamma_{\infty}} \cdot Q_{\Gamma_{0}}=\mathbb{1}$, thus $Q_{\Gamma_{t}}=Q_{\Gamma_{\infty}} \cdot Q_{\Gamma_{0}} \cdot Q_{\Gamma_{-\infty}}^{-1}$. What do we have? Notice that the quantity $W_{\Gamma_{0}}$ is the Wilson line calculated over the entire space, at time zero, and $W_{\Gamma_{t}}$ is the Wilson line calculated over the entire space, at some later time. The paths $\Gamma_{-\infty}$ and $\Gamma_{\infty}$ are simply the "evolution in time" of the spatial boundary. Taking as boundary conditions $C_{t}(t, \infty)=C_{t}(t,-\infty)$ we have $Q_{\Gamma_{\infty}}=Q_{\Gamma_{-\infty}} \equiv U(t)$, and we get an iso-spectral 
evolution for

$$
Q_{\Gamma_{t}}=P_{1} e^{-\int_{\Gamma_{t}} C_{x} d x}
$$

which implies that its eigenvalues are conserved in time, or equivalently $\operatorname{Tr}\left(Q_{\Gamma_{t}}^{n}\right)$. Also, it is clear that these charges (the eigenvalues) are not affected by gauge transformations.

\subsection{Generalisation of the Stokes theorem}

In the previous section it was shown how the (standard) non-Abelian Stokes theorem can be used, with the fact that the connection is flat, to build up conserved charges. These charges do not come from Noether's symmetries, being only revealed once the equations of motion are written as a zero curvature equation. The vanishing of the curvature is equivalent to the path independence of the Wilson line, and considering a loop as in figure (2.3), where we clearly separate the space and time, one can use this independence, with appropriate boundary conditions to get an iso-spectral evolution for the "spatial Wilson line" (i.e., the Wilson line calculated over the entire space, at certain time slice), whose eigenvalues are recognised as the conserved charges.

This construction works pretty well in the $(1+1)$-dimensional space-time, but this is not the case for higher dimensions, where neither the concept of integrability is understood. An idea to generalise this zero curvature formulation for $(d+1)$-dimensional theories was presented( $(6)$ in 1997 by Luiz Agostinho Ferreira, Joaquin Sánchez-Guillén and Orlando Alvarez. They noticed that the loop space looks like the adequate place to follow the steps mentioned above to get the conserved charges. Given a $(d+1)$ dimensional space-time $M$, the loop space $L M$ is defined by the set of mappings from the $(d-1)$ sphere $\left(S^{d-1}\right)$ to $M$, fixing the image of a point of the sphere, say, the north-pole, as the reference point $x_{\mathrm{R}}$ in $M$. The images of these maps are $(d-1)$ closed hyper-surfaces in $M$ based at $x_{\mathrm{R}}$, and each of them corresponds to a point in $L M$, while the hyper-volume, in between two such surfaces, corresponds to a path in $L M$. It is not difficult to see that in the case $d=1$ the loop space coincides with space-time. For $d=2$, the loops based at $x_{\mathrm{R}}$ in $M$ are points in $L M$, and the area of the surface between the infinitesimal loop around $x_{\mathrm{R}}$ and the loop forming the boundary at $\tau=2 \pi$ is a path in $L M$. For $d=3$ the surfaces in $M$ correspond to points in $L M$ and the 3-dimensional volumes in space-time to paths in the loop space.

So, following the approach in (6) the first step is to look for a charge operator that generalises $W_{\Gamma_{t}}$, the "spatial Wilson line". If the dimensionality of space increases then we expect the generalisation of the Wilson line to be related to a connection which is a differential 

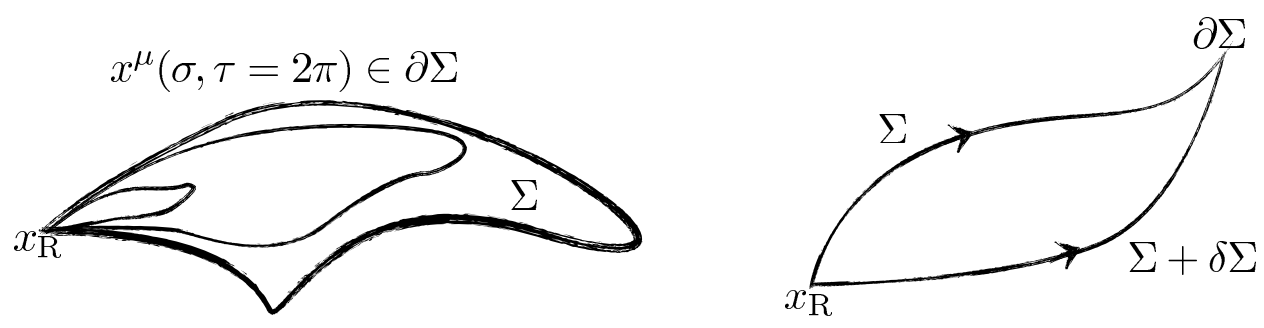

Figure 2.4 - On the left, a surface $\Sigma$ in $M$ is scanned with loops based at $x_{\mathrm{R}}$. On the right, this surface is represented in $L M$, where each loop in $M$ corresponds to a point and the surface from $x_{\mathrm{R}}$ to the boundary $\partial \Sigma$ is a path. A variation of this surface, leaving the boundary fixed, is also represented in $L M$.

form with higher degree. Notice that $W_{\Gamma_{t}}$ is the path-ordered exponential of a 1-form, which is something that we integrate over a 1-dimensional manifold, the space. For a space with 2 dimensions, we might expect a 2 -form, and so on. For a theory in $(d+1)$ dimensions their idea was to introduce a $d$-form field. So, one needs to find a way to define a generalisation of the Wilson line but now for a surface, and then for a volume, etc. which, in the loop space, correspond always to a path. Well, in fact this problem is more or less solved. The equation we are looking for is a generalisation of (2.1.6), where instead of $W$ we write $V$ (and call it the Wilson surface), and instead of $G_{\mu \nu}$, we introduce an anti-symmetric tensor $B_{\mu \nu}$ :

$$
\frac{d V}{d \tau}-V \int_{0}^{2 \pi} d \sigma W^{-1} B_{\mu \nu} W \frac{\partial x^{\mu}}{\partial \sigma} \frac{\partial x^{\nu}}{\partial \tau}=0
$$

with the initial condition being the constant $V_{\mathrm{R}}$, calculated on the infinitesimal surface around the reference point. This equation, when integrated in $\tau$, defines the Wilson surface $V$, in a 2-dimensional surface. How to do that was already discussed, and the result is formally written as

$$
V_{\Sigma}=V_{\mathrm{R}} \cdot P_{2} \exp \left(\int_{0}^{\tau} \mathcal{C} d \tau^{\prime}\right)
$$

where $\mathcal{C} \equiv \int_{0}^{2 \pi} d \sigma W^{-1} B_{\mu \nu} W \frac{\partial x^{\mu}}{\partial \sigma} \frac{\partial x^{\nu}}{\partial \tau^{\prime}}$

As discussed in (7), the quantity $\mathcal{C}$ appearing here can be understood as a connection in the loop space, so that the above $V$ is a direct generalisation of the Wilson line, indeed. As before, one could consider variations of the surface, and analyse how $V$ changes. Following the pattern for $W$, this variation will produce a new equation for $V$, such that if we consider the surface to be closed and the boundary of a volume then $V$ can be obtained by integrating (2.2.1) on that surface directly or by integrating this new equation along the volume from the infinitesimal closed surface around $x_{\mathrm{R}}$. The result from this new equation defines $V$ as a volume-ordered exponential of something that can be identified as the curvature of $\mathcal{C}$ in

$\S$ The 2 -form $B=\frac{1}{2} B_{\mu \nu} d x^{\mu} \wedge d x^{\nu}$ is not necessarily exact. 


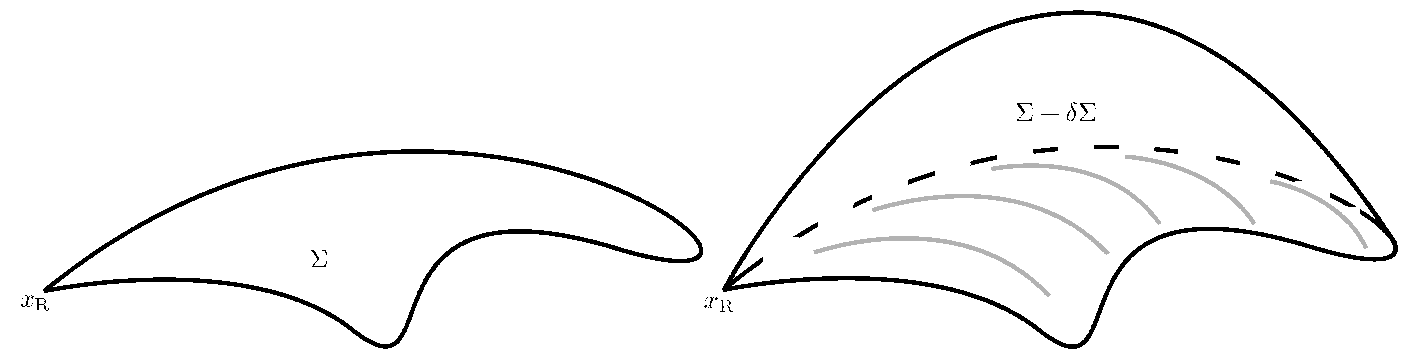

Figure 2.5 - The border of the surface is kept fix while performing the variation. When the surfaces are closed, the border is contracted to $x_{\mathrm{R}}$ and the initial surface $(\zeta=0)$ becomes the closed infinitesimal surface $\Sigma_{\mathrm{R}}$ while the final surface $(\zeta=2 \pi)$ becomes the boundary of a volume.

loop space, and the equivalence of the two ways of finding $V$ is the generalisation of Stokes theorem, as we shall discuss in a while.

Then, in (6) it is explained how to obtain local conditions that would make this curvature in loop space vanishes, and the Wilson surface becomes path independent, and we get a guide to define integrability for theories in $(2+1)$-dimensional space-time, and a way to calculate the conserved charges. Then, for theories in $(3+1)$ dimensions we generalise what was done here for $V$, to, say, a Wilson volume, and so on.

In this thesis we shall use basically the same ideas of (6) and (7), but instead of looking for a zero curvature in loop space we propose an integral equation of motion for the theory which is a consequence of the standard or generalised non-Abelian Stokes theorem and of the differential equations of the physical fields. Now that the goal was explained, let us discuss the calculations to generalise the non-Abelian Stokes theorem involving a 2-form connection.

As mentioned before, consider variations of the surface: $\Sigma \rightarrow \Sigma+\delta \Sigma$. Following the same reasoning used for the variation of the path $\Gamma$ in the case of the Wilson line, we take these variations to be in the direction perpendicular to the surface, and parametrise them with $\zeta \in[0,2 \pi]$, so defining a velocity $\frac{d x^{\mu}}{d \zeta}$. If we consider the surface to be closed, becoming the border of the volume $\Omega$, we notice that by continuously varying from the infinitesimal closed surface around the reference point $\Sigma_{\mathrm{R}}$, where the Wilson surface is $V_{\mathrm{R}}$, we scan $\Omega$ (see figure (2.5) ). The surface labelled by $\zeta=2 \pi$ corresponds to the boundary $\partial \Omega$. Each of this surfaces, in turn, are scanned with loops, based at $x_{\mathrm{R}}$. The calculation is long but straightforward and the result for the variation of the Wilson surface reads

IAlthough we shall be in most of the discussion interested in variations perpendicular to the surface, equation (2.2.3) holds for any kind of variation, including those tangent to the surface. 


$$
\begin{aligned}
& \delta V=\left(\int _ { 0 } ^ { 2 \pi } d \tau \int _ { 0 } ^ { 2 \pi } d \sigma V ( \tau ) \left\{W^{-1}\left(D_{\lambda} B_{\mu \nu}+D_{\mu} B_{\nu \lambda}+D_{\nu} B_{\lambda \mu}\right) W \frac{\partial x^{\mu}}{\partial \sigma} \frac{\partial x^{\nu}}{\partial \tau} \delta(22 \lambda .2 .3\right.\right. \\
& -\int_{0}^{\sigma} d \sigma^{\prime}\left[B_{\kappa \lambda}^{W}\left(\sigma^{\prime}\right)-G_{\kappa \lambda}^{W}\left(\sigma^{\prime}\right), B_{\mu \nu}^{W}(\sigma)\right] \times \\
& \left.\left.\times \frac{\partial x^{\kappa}}{\partial \sigma^{\prime}} \frac{\partial x^{\mu}}{\partial \sigma}\left(\frac{\partial x^{\lambda}}{\partial \tau}\left(\sigma^{\prime}\right) \delta x^{\nu}(\sigma)-\delta x^{\lambda}\left(\sigma^{\prime}\right) \frac{\partial x^{\nu}}{\partial \tau}(\sigma)\right)\right\} V^{-1}(\tau)\right) \cdot V
\end{aligned}
$$

where $D_{\mu} \star=\partial_{\mu} \star+\left[C_{\mu}, \star\right]$, and we use the notation $X^{W} \equiv W^{-1} X W$. Again, since the variation is with respect to the parameter $\zeta$, this equation can be written as an equation in that parameter:

$$
\frac{d V}{d \zeta}-\mathcal{K} V=0
$$

where

$$
\begin{aligned}
\mathcal{K}= & \int_{0}^{2 \pi} d \tau \int_{0}^{2 \pi} d \sigma V(\tau)\left\{W^{-1}\left(D_{\lambda} B_{\mu \nu}+D_{\mu} B_{\nu \lambda}+D_{\nu} B_{\lambda \mu}\right) W \frac{\partial x^{\mu}}{\partial \sigma} \frac{\partial x^{\nu}}{\partial \tau} \frac{\partial x^{\lambda}}{\partial \zeta}\right. \\
& -\int_{0}^{\sigma} d \sigma^{\prime}\left[B_{\kappa \lambda}^{W}\left(\sigma^{\prime}\right)-G_{\kappa \lambda}^{W}\left(\sigma^{\prime}\right), B_{\mu \nu}^{W}(\sigma)\right] \times \\
& \left.\times \frac{\partial x^{\kappa}}{\partial \sigma^{\prime}} \frac{\partial x^{\mu}}{\partial \sigma}\left(\frac{\partial x^{\lambda}}{\partial \tau}\left(\sigma^{\prime}\right) \frac{\partial x^{\nu}}{\partial \zeta}(\sigma)-\frac{\partial x^{\lambda}}{\partial \zeta}\left(\sigma^{\prime}\right) \frac{\partial x^{\nu}}{\partial \tau}(\sigma)\right)\right\} V^{-1}(\tau) .
\end{aligned}
$$

The quantities $W$ and $V$ inside $\mathcal{K}$ are calculated from (2.1.1) and (2.2.1) respectively. $W$ is integrated along the loops scanning each surface, while $V$ is calculated on each of the surfaces scanning the volume. The integration of (2.2.4) is done similarly as those explained before. Now we have a volume-ordering which is represented by the $P_{3}$. The result can be written formally as

$$
V_{\Sigma_{\mathrm{c}}}=P_{3} \exp \left(\int_{0}^{2 \pi} \mathcal{K} d \zeta\right) \cdot V_{\mathrm{R}}
$$

The equality between the Wilson surface obtained in (2.2.5) and that in (2.2.2) (for $\Sigma_{\mathrm{c}}=\partial \Omega$ ) is the statement of the generalised non-Abelian Stokes theorem:

$$
V_{\mathrm{R}} \cdot P_{2} \exp \left(\oint_{\partial \Omega} \mathcal{C} d \tau\right)=P_{3} \exp \left(\int_{\Omega} \mathcal{K} d \zeta\right) \cdot V_{\mathrm{R}}
$$

The idea now is to set the quantities $B_{\mu \nu}$ and $C_{\mu}$ in terms of physical fields, whose dynamics are governed by differential equations, and we state that the Stokes theorem is the integral equation of motion, in the sense that when the hyper-volume becomes infinitesimal one recovers the differential equations. We shall formulate the integral equations using (2.1.8) and (2.2.6). In fact, the general form of the integral equation in the $(d+1)$-dimensional space-time $M$ is

$$
P_{d-1} e^{\int_{\partial \Omega} \mathcal{A}}=P_{d} e^{\int_{\Omega} \mathcal{F}}
$$


where $\Omega$ is a sub-manifold of $M$ with dimension $d$, and $\partial \Omega$ its boundary. The quantities $\mathcal{A}$ and $\mathcal{F}$ are defined from $(d-1)$-forms and $d$-forms respectively, in terms of the physical fields.

Now, if we consider $\Omega$ to be closed, the I.h.s becomes $\mathbb{1}$ and we get $P_{d} e^{\int_{\Omega_{\mathrm{c}}} \mathcal{F}}=\mathbb{1}$. This relation will lead us to a conservation law, in a similar way of that we saw appearing from the zero curvature formulation in $(1+1)$ dimensions, in terms of the Wilson line: splitting the space-time into space and time and with appropriate boundary conditions we find an isospectral evolution for the operator $P_{d} e^{\int_{\Omega_{\text {space }}} \mathcal{F}}$ calculated over the space, at some time slice. This will give us the conserved charges.

The main difficulty in this approach, as in the construction of the zero curvature representation in 2-dimensions, is to find the appropriate way to write $C_{\mu}$ and $B_{\mu \nu}$ in terms of the physical fields, in a way that the integral equations will imply the differential equations when $\Omega$ becomes infinitesimal. In this thesis we show how this can be done for gauge theories: Chern-Simons and Yang-Mills in $(2+1)$ dimensions and Yang-Mills in $(3+1)$ dimensions. We explored some configurations of the latter case such as the monopoles, dyons and also in the euclidean sector with the merons and instantons, calculating their charges, which arise from our construction. 


\section{CHAPTER 3}

\section{Integral formulation of theories in}

\section{$2+1$ dimensions}

In this chapter we show how the standard non-Abelian Stokes theorem (2.1.8) provides an integral version of Chern-Simons and Yang-Mills equations in three dimensions. These equations lead us to some conserved quantities in a very natural way. The construction is very similar to what is usually done in the 2-dimensional space-time, but now the paths in figure (2.3) are replaced by surfaces, which, due to the construction in loop space, are scanned by loops. The physics of the problem show us the boundary conditions, which are in fact precisely what is needed to obtain an iso-spectral evolution for the charge operator that generalises the "spatial Wilson surface" as described above.

\subsection{The integral equations of Chern-Simons theory}

We consider a 3-dimensional Minkowski space-time $M$. The field $C_{\mu}(\mu=0,1,2)$ in (2.1.8) is written in terms of the gauge field $A_{\mu}$ (containing the physical degrees of freedom) as $C_{\mu}=i e A_{\mu} ; e$ is the gauge coupling constant. So, the curvature of $C$ reads $G_{\mu \nu}=$ $\partial_{\mu} C_{\nu}-\partial_{\nu} C_{\mu}+\left[C_{\mu}, C_{\nu}\right]=i e F_{\mu \nu}$, where $F_{\mu \nu}$ is the field strength. The differential ChernSimons equation is given by $F_{\mu \nu}=\frac{1}{\kappa} \epsilon_{\mu \nu \rho} J^{\rho}$, where $J^{\mu}$ is the matter current, and $\kappa$ is a coupling constant. Using that we obtain $G_{\mu \nu}=\frac{i e}{\kappa} \widetilde{J}_{\mu \nu}$, where $\widetilde{J}_{\mu \nu}$ is the Hodge dual of $J^{\mu}$. Finally, we have the I.h.s and the r.h.s of (2.1.8) in terms of the physical fields: the first is related to the gauge field $A_{\mu}$ while the second, to the matter field $J_{\mu}$. Then, the integral equation reads

$$
P_{1} \exp \left(-i e \oint_{\partial \Sigma} A_{\mu} d x^{\mu}\right)=P_{2} \exp \left(\frac{i e}{\kappa} \int_{\Sigma} W^{-1} \widetilde{J}_{\mu \nu} W d x^{\mu} d x^{\nu}\right) .
$$

One notice that the integration constants $W_{\mathrm{R}}$ do not appear in the above equation. This is because the Stokes theorem is now promoted from a mathematical identity to a physical equa- 
tion, and therefore we require its gauge covariance, which in turn implies that the integration constants must be in the centre of the group, as we now show, and therefore they become irrelevant or factorisable from (3.1.1).

The r.h.s of the above equation is $V$, calculated from (2.2.1), where in $\mathcal{C}$ we use $B_{\mu \nu}=$ $\frac{i e}{\kappa} \widetilde{J}_{\mu \nu}$. This quantity, $\mathcal{C}$, is integrated along the loop, starting and ending at the same point $x_{\mathrm{R}}$. Under a gauge transformation $A_{\mu} \rightarrow h A_{\mu} h^{-1}+\frac{i}{e} \partial_{\mu} h h^{-1}$ the field strength transforms as $F_{\mu \nu} \rightarrow h F_{\mu \nu} h^{-1}$, and similarly the dual of the current (by consistency of the Chern-Simons equation). For a loop, the Wilson line transforms as $W \rightarrow h\left(x_{\mathrm{R}}\right) \cdot W \cdot h^{-1}\left(x_{\mathrm{R}}\right)$ and it is easy to see then that $\mathcal{C} \rightarrow \mathcal{C}^{\prime}=h \mathrm{Ch}^{-1}$. Now, we suppose that $V$ defined by (2.2.1) becomes $V^{\prime}$, satisfying $\frac{d V^{\prime}}{d \tau}-V^{\prime} \mathcal{C}^{\prime}=0$. Then, one finds that under a gauge transformation $V^{\prime}=h\left(x_{\mathrm{R}}\right) \cdot V h^{-1}\left(x_{\mathrm{R}}\right)$.

Now, equation (2.2.1) defines $V$ up to a constant group element on the left, i.e., if $V$ is a solution of (2.2.1), then $V^{k}=k \cdot V$ is a solution too. So, under a gauge transformation $V^{k}$ transforms like $V^{k} \rightarrow k \cdot h \cdot V \cdot h^{-1}$, which, on the other hand, is $V^{k} \rightarrow h \cdot k \cdot V \cdot h^{-1}$. Consistency implies that $k \cdot h=h \cdot k$, so $k$ belongs to the centre of the group. Equivalently, the I.h.s. of $(3.1 .1)$ is $W$, a solution of (2.1.1) with $C_{\mu}=i e A_{\mu}$. This is defined up to a constant element on the right, so if $W$ is a solution, $W^{q}=W \cdot q$ is a solution as well. A completely equivalent reasoning as that given for $V^{k}$ holds for $W^{q}$. Then, we conclude that the same construction applies to the case where $k$ and $q$ correspond to $W_{\mathrm{R}}$, and that is how it can be ignored in the integral equation.

The integral equation is defined naturally on the loop space $L \Sigma$; a loop based at $x_{\mathrm{R}}$ on the border $\partial \Sigma$ corresponds to a point in $L \Sigma$, and the surface $\Sigma$ corresponds to a path. If we change the reference point or de scanning the integral equation will transform "covariantly", i.e., both sides will change accordingly. A change on the scanning of the surface with loops (i.e., if one chooses a new way of constructing the loops scanning $\Sigma$ ) will change the path in $L \Sigma$ but the physical surface remains the same.

Now, we verify that (3.1.1) is indeed the integral equation of Chern-Simons theory, by showing that when $\Sigma$ is an infinitesimal surface, the differential equation is recovered. So, let $\Sigma$ be an infinitesimal rectangle of sides $\delta x \delta y$, the reference point being the origin of the Cartesian system. Lets expand the I.h.s around this point. We remember that the quantity $P_{1} \exp \left(-i e \oint_{\partial \Sigma} A_{\mu} d x^{\mu}\right)$ is a solution of equation (2.1.1), with $C_{\mu}=i e A_{\mu}$, along the curve $\partial \Sigma$. So, we can consider the "infinitesimal version" of this equation as $W(\sigma+\delta \sigma)=W(\sigma)-$ $C_{\mu}(\sigma) \delta x^{\mu} W(\sigma)$ and calculate it iteratively along the rectangle. For instance, when we go from

* Regardless the labels $x$ and $y$, it does not mean we are in space; on the contrary, the surface is in space-time. 
$x_{\mathrm{R}}$ to $\delta x$ we get $W\left(x_{\mathrm{R}}+\delta x\right)=W_{\mathrm{R}}-C_{\mu}\left(x_{\mathrm{R}}\right) \delta x^{\mu} W_{\mathrm{R}}$. Next, $W\left(x_{\mathrm{R}}+\delta x+\delta y\right)=W\left(x_{\mathrm{R}}+\delta x\right)-$ $C_{\mu}\left(x_{\mathrm{R}}+\delta x\right) \delta y^{\mu} W\left(x_{\mathrm{R}}+\delta x\right)$, and we use the previous result and taylor expand the connection; everything up to second order. Doing that, and paying attention to the signs that change when we go in the negative direction of the axis, the result appears with not much difficulty: $P_{1} \exp \left(-i e \oint_{\partial \Sigma} A_{\mu} d x^{\mu}\right) \approx 1+i e F_{\mu \nu} \delta x^{\mu} \delta y^{\nu}$, no sums in $\mu, \nu$. For the r.h.s, it is direct, if we keep things up to second order: $P_{2} \exp \left(\frac{i e}{\kappa} \int_{\Sigma} W^{-1} \widetilde{J}_{\mu \nu} W d x^{\mu} d x^{\nu}\right) \approx \mathbb{1}+\frac{i e}{\kappa} \widetilde{J}_{\mu \nu} \delta x^{\mu} \delta y^{\nu}$. This came from the "infinitesimal version" of (2.2.1), $V(\tau+\delta \tau)=V(\tau)+V(\tau) \mathcal{C}(\tau) \delta \tau$, using the fact that we only need it at the reference point since if we go any further a higher order term will appear in the Taylor expansiont ${ }^{t}$. Of course, due to the infinitesimal character of $\Sigma$ the integration becomes the integrand times the area. Finally, we clearly see that the equality of these expansions implies the Chern-Simons equation.

Our next step is to build up the conserved charges. In order to do so we consider the surface to be closed, $\Sigma_{c}$, so that it has no border and as a consequence of the integral equation we get

$$
V_{\Sigma_{\mathrm{c}}}=P_{2} \exp \left(\frac{i e}{\kappa} \int_{\Sigma_{\mathrm{c}}} W^{-1} \widetilde{J}_{\mu \nu} W d x^{\mu} d x^{\nu}\right)=\mathbb{1},
$$

where we emphasize the fact that this quantity is a solution of (2.2.1), with $B_{\mu \nu}=\frac{i e}{\kappa} \widetilde{J}_{\mu \nu}$.

The surface $\Sigma_{c}$ can be considered as formed by two other surfaces (see figure (3.1)), $\Sigma_{\mathrm{c}}=\Sigma_{1} \circ \Sigma_{2}^{-1}$, and the intersection of them is a certain loop $\Gamma$. Then the above equation implies $V_{\Sigma_{1}}=V_{\Sigma_{2}}$. This equation is expressing the fact that the Wilson surface $V$ linking the infinitesimal loop around $x_{\mathrm{R}}$ to the loop $\Gamma$ is surface independent, or, in loop space, path independent. As we did in the 2-dimensional case we split space-time into space and time. The surface $\Sigma_{1}$ is composed by a purely spatial part at $t=0$, the disk $D_{\infty}^{(0)}$ that extends from the reference point to the whole space; and there is a second part which is the the boundary $\partial D_{\infty}^{(0)}=S_{\infty}^{1}$ (a point in $L M$, and a loop in $M$ ) moving forward until a time $t>0: S_{\infty}^{1} \times \mathbb{R}$. Thus, $V_{\Sigma_{1}}=V_{D_{\infty}^{(0)}} \cdot V_{S_{\infty}^{1} \times \mathbb{R}}$. The surface $\Sigma_{2}$ is also composed by two parts. The first part is the infinitesimal loop around $x_{\mathrm{R}}$ moving forward in time, $S_{\mathrm{R}}^{1} \times \mathbb{R}$, and the second part is the whole space, $D_{\infty}^{(t)}$. Thus $V_{\Sigma_{2}}=V_{S_{R}^{1} \times \mathbb{R}} \cdot V_{D_{\infty}^{(t)}}$. Then, surface independence implies

$$
V_{D_{\infty}^{(0)}} \cdot V_{S_{\infty}^{1} \times \mathbb{R}}=V_{S_{\mathbb{R}}^{1} \times \mathbb{R}} \cdot V_{D_{\infty}^{(t)}}
$$

These quantities are calculated from (2.2.1), on each surface, scanned by loops based at $x_{\mathrm{R}}$. Consider the scanning of $\Sigma_{1}$. We start by scanning $D_{\infty}^{(0)}$ with the loops staring and ending at $x_{\mathrm{R}}$. Then, we have to scan the cylinder $S_{\infty}^{1} \times \mathbb{R}$, which is at spatial infinity. Basically we keep going around the same spatial circle $S_{\infty}^{1}$, but at each lap we move forward in time, so

†We use $W \approx\left(1-C_{\mu}\left(x_{\mathrm{R}}\right) \delta x^{\mu}\right)$, its inverse and $J^{\rho}\left(x_{\mathrm{R}}+\delta x\right) \approx J^{\rho}\left(x_{\mathrm{R}}\right)+\partial_{\mu} J^{\rho}\left(x_{\mathrm{R}}\right) \delta x^{\mu}$ 

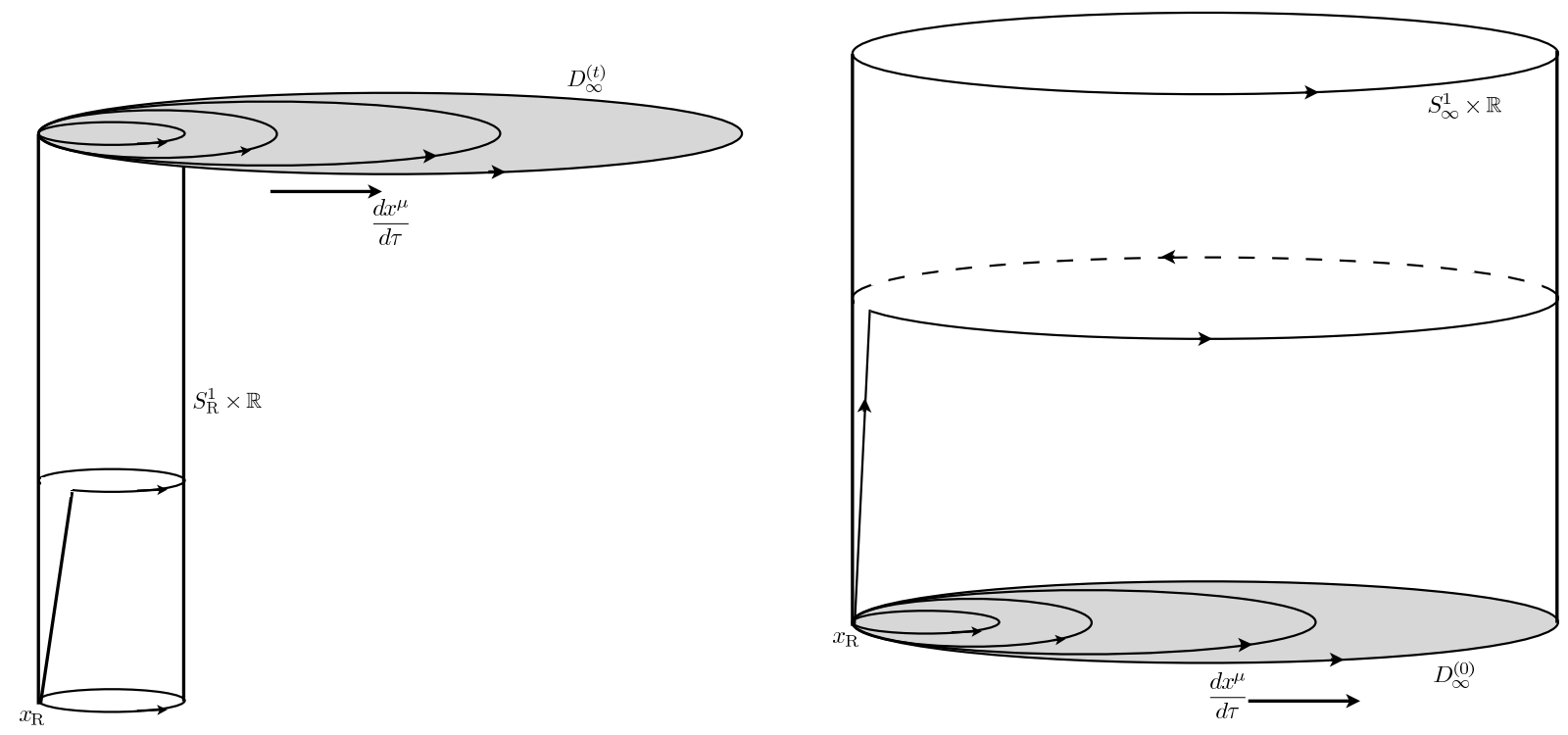

Figure 3.1 - The surface independence of $V$ means that it can be calculated from the infinitesimal loop around $x_{\mathrm{R}}$ (the initial point in loop space) to the boundary loop $S_{\infty}^{1}$ (the final point in loop space) using any of the two surfaces (paths in loop space) presented here.

there is always a leg linking $x_{\mathrm{R}}$ with the circle above. The integration of (2.2.1) depends on the integration of $\mathcal{C}=\frac{i e}{\kappa} \oint d \sigma W^{-1} \widetilde{J}_{\mu \nu} W \frac{\partial x^{\mu}}{\partial \sigma} \frac{\partial x^{\nu}}{\partial \tau}$ in $\tau$, on the spatial surface, so it will depend on the integration of $J_{0}$ over the area of the disk. Then, if we set that at spatial infinity $J_{0} \sim \frac{1}{r^{2+\delta}}$, with $\delta>0$ and $r=\sqrt{x_{1}^{2}+x_{2}^{2}}$, this integration will vanish, and $V_{S_{\infty}^{1} \times \mathbb{R}}=V_{\mathrm{R}}$. Also, due to the fact that $S_{\mathrm{R}}^{1}$ has an infinitesimal radius (it is just a point, basically), we have that $V_{S_{\mathrm{R}}^{1} \times \mathbb{R}}=V_{\mathrm{R}}$. So, remembering that $V_{\mathrm{R}}$ is in the centre of the group one gets

$$
V_{D_{\infty}^{(t)}}=V_{D_{\infty}^{(0)}}
$$

which relates the "spatial Wilson surfaces" at different times. But, that is not all. One has to keep in mind that all Wilson surfaces are calculated by scanning the surface with a family of loops based at the fixed reference point $x_{\mathrm{R}}$, in the border of space at $t=0$. If we want a real conservation law we need to be able to calculate the "spatial Wilson surface" from the reference point at any given time slice, independently. So, we consider the reference point at time $t, x_{\mathrm{R}}^{t}$, in the border of $D_{\infty}^{(t)}$. Then, we can decompose every loop we use to scan $\Sigma_{2}$ into three parts: the "leg" going forward in time from $x_{\mathrm{R}}$ to $x_{\mathrm{R}}^{t}$, then the part that goes around the disk, and the "leg" coming back from $x_{\mathrm{R}}^{t}$ to $x_{\mathrm{R}}$; consequently the Wilson line will be decomposed as well. In particular, when we are somewhere in the loop going around the disk, $W\left(\sigma, x_{\mathrm{R}}\right)=W\left(\sigma, x_{\mathrm{R}}^{t}\right)$. $W\left(x_{\mathrm{R}}^{t}, x_{\mathrm{R}}\right)$, and we have $\mathcal{C}=\int_{0}^{2 \pi} d \sigma W^{-1}\left(x_{\mathrm{R}}^{t}, x_{\mathrm{R}}\right) \cdot W^{-1}\left(\sigma, x_{\mathrm{R}}\right) B_{\mu \nu} W\left(\sigma, x_{\mathrm{R}}\right) \cdot W\left(x_{\mathrm{R}}^{t}, x_{\mathrm{R}}\right)=$ $W^{-1}\left(x_{\mathrm{R}}^{t}, x_{\mathrm{R}}\right) \mathcal{C} W\left(x_{\mathrm{R}}^{t}, x_{\mathrm{R}}\right)$, where $W\left(x_{\mathrm{R}}^{t}, x_{\mathrm{R}}\right)$ is calculated using (2.1.1). So, lets call now $V_{D_{\infty}^{(t)}}^{t}$ the Wilson surface on the disk $D_{\infty}^{(t)}$ obtained from the reference point $x_{\mathrm{R}}^{t}$, and similarly, $V_{D_{\infty}^{(t)}}$ the Wilson surface on the disk $D_{\infty}^{(t)}$ obtained from the reference point $x_{\mathrm{R}}$. Then we see that 
integration of (2.2.4) gives, using $\mathcal{C}$ as above, $V_{D_{\infty}^{(t)}}^{t}=W\left(x_{\mathrm{R}}^{t}, x_{\mathrm{R}}\right) \cdot V_{D_{\infty}^{(t)}} \cdot W^{-1}\left(x_{\mathrm{R}}^{t}, x_{\mathrm{R}}\right)$. Finally, the Wilson surface independence implies in the iso-spectral evolution

$$
V_{D_{\infty}^{(t)}}^{t}=W\left(x_{\mathrm{R}}^{t}, x_{\mathrm{R}}\right) \cdot V_{D_{\infty}^{(0)}} \cdot W^{-1}\left(x_{\mathrm{R}}^{t}, x_{\mathrm{R}}\right)
$$

and therefore the eigenvalues of the operator

$$
V_{D_{\infty}^{(t)}}^{t}=P_{2} \exp \left(\frac{i e}{\kappa} \int_{D_{\infty}^{(t)}} W^{-1} \widetilde{J}_{\mu \nu} W d x^{\mu} d x^{\nu}\right)=P_{1} \exp \left(-i e \oint_{S_{\infty}^{1,(t)}} A_{\mu} d x^{\mu}\right)
$$

are the conserved charges.

As we discussed, under a gauge transformation this operator changes as $V_{D_{\infty}^{(t)}}^{t} \rightarrow h\left(x_{\mathrm{R}}\right)$. $V_{D_{\infty}^{(t)}}^{t} h^{-1}\left(x_{\mathrm{R}}\right)$, and therefore the charges, which are the eigenvalues, remain invariant. Also, if one decides to change the scanning, i.e., the parametrisation of the surface, the path independence in loop space (3.1.2) shows that the charge operator above remains the same. Moreover, by changing the reference point $x_{\mathrm{R}}$ to some other point on the border of the disk, the charge operator will change by conjugation with the Wilson line joining the two points, and clearly, the charges remain the same. Since the reference point is at the border of the disk, at infinity, our boundary condition says that $F_{\mu \nu} \sim 0$, and therefore $A_{\mu}$ becomes flat, so $W$ joining the two different reference points is independent of the curve we choose.

\subsection{The integral equations of $(2+1)$-dimensional Yang- Mills theory}

We start by choosing the connection $C_{\mu}$ in terms of the physical fields, in this case, the Yang-Mills field $A_{\mu}: C_{\mu}=i e\left(A_{\mu}+\beta \widetilde{F}_{\mu}\right)$, where $\widetilde{F}_{\mu}=\frac{1}{2} \epsilon_{\mu \nu \rho} F^{\nu \rho}$ is the Hodge dual of the field strength $F_{\mu \nu}=\partial_{\mu} A_{\nu}-\partial_{\nu} A_{\mu}+i e\left[A_{\mu}, A_{\nu}\right]$, and $\beta \in \mathbb{C}$ is an arbitrary parameter. Notice that this choice is compatible with the fact that $C_{\mu}$ is a connection, i.e., given that under a gauge transformation $A_{\mu} \rightarrow h A_{\mu} h^{-1}+\frac{i}{e} \partial_{\mu} h h^{-1}$, and $F_{\mu \nu} \rightarrow h F_{\mu \nu} h^{-1}$, the combination above implies that $C_{\mu} \rightarrow h C_{\mu} h^{-1}-\partial_{\mu} h h^{-1}$, as expected. Now, in the r.h.s of the Stokes theorem we have the curvature of $C_{\mu}, G_{\mu \nu}$. The differential equation of Yang-Mills read $D_{\nu} F^{\nu \mu}=J^{\mu}$ and $D_{\mu} \widetilde{F}^{\mu}=0$, where $D_{\mu} \star=\partial_{\mu} \star+\left[A_{\mu}, \star\right]$ and $J_{\mu}$ is the matter current. Thus, calculating $G_{\mu \nu}$ and using the differential equations one gets $G_{\mu \nu}=i e\left(F_{\mu \nu}-\beta \widetilde{J}_{\mu \nu}+i e \beta^{2}\left[\widetilde{F}_{\mu}, \widetilde{F}_{\nu}\right]\right)$, and finally the integral equation of Yang-Mills theory reads

$$
P_{1} e^{-i e \oint_{\partial \Sigma}\left(A_{\mu}+\beta \widetilde{F}_{\mu}\right) d x^{\mu}}=P_{2} e^{i e \int_{\Sigma} W^{-1}\left(F_{\mu \nu}-\beta \widetilde{J}_{\mu \nu}+i e \beta^{2}\left[\widetilde{F}_{\mu}, \widetilde{F}_{\nu}\right]\right) W d x^{\mu} d x^{\nu}} .
$$


Now we need to check if when $\Sigma$ is considered to be infinitesimal, the differential equations are recovered. Using the same reasoning as before we consider $\Sigma$ to be an infinitesimal rectangular surface and expand each side of the integral equation on it, which gives: $P_{1} e^{-i e \oint_{\partial \Sigma}\left(A_{\mu}+\beta \widetilde{F}_{\mu}\right) d x^{\mu}} \approx \mathbb{1}+i e\left(F_{\mu \nu}+\beta\left(D_{\mu} \widetilde{F}_{\nu}-D_{\nu} \widetilde{F}_{\mu}\right)+i e \beta^{2}\left[\widetilde{F}_{\mu}, \widetilde{F}_{\nu}\right]\right) \delta x^{\mu} \delta y^{\nu}$ for the I.h.s and, $P_{2} e^{i e \int_{\Sigma} W^{-1}\left(F_{\mu \nu}-\beta \widetilde{J}_{\mu \nu}+i e \beta^{2}\left[\widetilde{F}_{\mu}, \widetilde{F}_{\nu}\right]\right) W d x^{\mu} d x^{\nu}} \approx \mathbb{1}+i e\left(F_{\mu \nu}-\beta \widetilde{J}_{\mu \nu}+i e \beta^{2}\left[\widetilde{F}_{\mu}, \widetilde{F}_{\nu}\right]\right)$ for the r.h.s. Clearly, equating both terms we get $D_{\nu} F^{\nu \mu}=J^{\mu}$.

The conserved charges are constructed in an analogous way as we did for the Chern-Simons theory. For a closed surface we get that

$$
V_{\Sigma_{\mathrm{c}}}=P_{2} e^{i e \int_{\Sigma_{\mathrm{c}}} W^{-1}\left(F_{\mu \nu}-\beta \widetilde{J}_{\mu \nu}+i e \beta^{2}\left[\widetilde{F}_{\mu}, \widetilde{F}_{\nu}\right]\right) W d x^{\mu} d x^{\nu}}=\mathbb{1}
$$

expressing the surface independence of the Wilson surface joining the infinitesimal loop around $x_{\mathrm{R}}$ and $S_{\mathrm{R}}^{1}$, if we take $\Sigma_{\mathrm{c}}$ as before. The next step is to look for the iso-spectral evolution of the "spatial Wilson surface". The difference is that now the boundary conditions are defined not just in terms of the matter current, but also in terms of the Yang-Mills field strength, since in $V_{\Sigma_{\mathrm{c}}}$ above both quantities appear. So, we consider $J_{0} \sim \frac{1}{r^{2+\delta}}$ and also $F_{\mu \nu} \sim \frac{1}{r^{2+\varepsilon}}$, with $\delta$ and $\varepsilon$ positive, at $r \rightarrow \infty$, then one gets the charge operator

$$
V_{D_{\infty}^{(t)}}^{t}=P_{2} e^{i e \int_{D_{\infty}^{(t)}} W^{-1}\left(F_{\mu \nu}-\beta \widetilde{J}_{\mu \nu}+i e \beta^{2}\left[\widetilde{F}_{\mu}, \widetilde{F}_{\nu}\right]\right) W d x^{\mu} d x^{\nu}}=P_{1} e^{-i e \oint_{S_{\infty}^{1,(t)}}\left(A_{\mu}+\beta \widetilde{F}_{\mu}\right) d x^{\mu}}
$$

whose eigenvalues are the conserved charges, with the same nice features under gauge transformation and re-parametrisation, as discussed before. 


\section{CHAPTER 4}

\section{The integral Yang-Mills equation in $3+1$ dimensions}

Here we present the construction of the integral equations of Yang-Mills theory in 4dimensional space-time, and derive the conserved charges from our construction in loop space. The generalised Stokes theorem is used, and also the standard one, in the case of the self-dual sector. Moreover the standard Stokes theorem can appear as an identity given some conditions, originating another operator whose eigenvalues are also conserved charges. We calculate the charges for some configurations of the theory namely monopoles, dyons, instantons and merons.

\subsection{The full Yang-Mills integral equation}

Let $M$ be the 4-dimensional, simply-connected, Minkowski space-time manifold. Suppose $\Omega$ to be a 3-dimensional sub-manifold in $M$, i.e., a volume. According to the ideas we discussed before, the loop space we are interested in is that of the maps from the 2-sphere into $M$, so that a volume such as $\Omega$ in $M$ becomes a path in loop space and each point in this path in loop space is a surface in $M$. In order to proceed like this we define a reference point $x_{\mathrm{R}}$ on the border of $\Omega$, and there we build an infinitesimal closed surface $\Sigma_{R}$, which is changed continuously (picture someone blowing a balloon from $x_{R}$ ) until reaches the border of $\Omega$. Each of these surfaces is scanned by a family of loops, also based at $x_{\mathrm{R}}$. On the surface we calculate the Wilson surface defined by (2.2.1), and the Wilson lines along the loops are calculated from (2.1.1). We shall construct the integral equations of Yang-Mills from the generalised Stokes theorem (2.2.6) and the differential equations $D_{\nu} F^{\nu \mu}=J^{\mu}, D_{\nu} \widetilde{F}^{\nu \mu}=0$, where $J_{\mu}$ stands for the matter current and $\widetilde{F}_{\mu \nu}=\frac{1}{2} \epsilon_{\mu \nu \rho \lambda} F^{\rho \lambda}$ is the Hodge dual of the field strength. For convenience we introduce the notation $\mathcal{K}=\int_{0}^{2 \pi} d \tau V \mathcal{J} V^{-1}$, in $\mathcal{K}$ of (2.2.4). The first step is to define $\mathcal{C}$ and $\mathcal{J}$ in terms of the Yang-Mills field. We set $B_{\mu \nu}$ in (2.2.1) as $B_{\mu \nu}=\alpha F_{\mu \nu}+\beta \widetilde{F}_{\mu \nu}$, where $\alpha$ and $\beta$ are arbitrary constants in $\mathbb{C}$. Then it is not difficult to 
see that this choice and the differential equations give us

$$
\begin{aligned}
\mathcal{J}= & \int_{0}^{2 \pi} d \sigma\left\{W^{-1}\left(i e \beta \widetilde{J}_{\mu \nu \lambda}\right) W \frac{\partial x^{\mu}}{\partial \sigma} \frac{\partial x^{\nu}}{\partial \tau} \frac{\partial x^{\lambda}}{\partial \zeta}\right. \\
& +\int_{0}^{\sigma} d \sigma^{\prime}\left[(\alpha-1) F_{\kappa \lambda}^{W}\left(\sigma^{\prime}\right)+\beta \widetilde{F}_{\kappa \lambda}^{W}\left(\sigma^{\prime}\right),\left(\alpha F_{\mu \nu}^{W}(\sigma)+\beta \widetilde{F}_{\mu \nu}^{W}(\sigma)\right)\right] \times \\
& \left.\times \frac{\partial x^{\kappa}}{\partial \sigma^{\prime}} \frac{\partial x^{\mu}}{\partial \sigma}\left(\frac{\partial x^{\lambda}}{\partial \tau}\left(\sigma^{\prime}\right) \frac{\partial x^{\nu}}{\partial \zeta}(\sigma)-\frac{\partial x^{\lambda}}{\partial \zeta}\left(\sigma^{\prime}\right) \frac{\partial x^{\nu}}{\partial \tau}(\sigma)\right)\right\} .
\end{aligned}
$$

where $J^{\rho}=\frac{1}{3 !} \epsilon^{\rho \mu \nu \lambda} \widetilde{J}_{\mu \nu \lambda}$. Now we can plug that $B_{\mu \nu}$ in $\mathcal{C}$, and get the I.h.s of Stokes theorem (2.2.6), and that $\mathcal{J}$ into $\mathcal{K}$ to get the r.h.s. With the integration constants in the centre of the group, following the arguments given before, we write down the integral Yang-Mills equation:

$$
P_{2} \exp \left(i e \int_{\partial \Omega} W^{-1}\left(\alpha F_{\mu \nu}+\beta \widetilde{F}_{\mu \nu}\right) W d x^{\mu} d x^{\nu}\right)=P_{3} \exp \left(\int_{\Omega} d \zeta d \tau V \mathcal{J} V^{-1}\right) .
$$

Our next step is to show that when $\Omega$ is an infinitesimal cube of sides $\delta x, \delta y$ and $\delta z$, the differential equations are recovered. We start by letting the reference point to be at one of the corners of the cube (see figure (4.1)), such that the sides form a Cartesian frame. We need to evaluate each side of the integral equation above on that cube, up to the first non-trivial order. The I.h.s of the integral equation is defined on the surface of the (closed) cube. This surface is scanned with loops, based at $x_{\mathrm{R}}$. The bottom plane of the cube is "scanned" with one loop, going around the border and coming back to $x_{\mathrm{R}}$. The signs of $\frac{d x^{\mu}}{d \sigma}$ and $\frac{d x^{\mu}}{d \tau}$ are positive. Of course, the cube being infinitesimal so that only one loop is required, makes this harder to see. So, for the sake of visualisation we imagine this cube as a finite surface. Then, $\frac{d x^{\mu}}{d \tau}$ positive means that we start from the reference point going to the border, while for $\frac{d x^{\mu}}{d \tau}$ negative we start from the border, going to the reference point. After "scanning" the infinitesimal bottom surface we move along a straight line in the $\delta z$ direction, until reach the top surface. Then, we continue moving around the border of that surface, but now with a negative $\frac{d x^{\mu}}{d \tau}$, going back to the reference point. So, we evaluate $P_{2} \exp \left(i e \int_{\partial \Omega} W^{-1}\left(\alpha F_{\mu \nu}+\beta \widetilde{F}_{\mu \nu}\right) W d x^{\mu} d x^{\nu}\right)$ in two parts: at the bottom surface and at the top, and then, we repeat this calculation for the other 2 pairs of surfaces of the cube. At the bottom surface we calculate the integrand at the reference point. If we take it at any other point then the contributions will be of higher order; notice that $W\left(x_{\mathrm{R}}+\delta x\right) \sim 1-i e A_{\mu}\left(x_{\mathrm{R}}\right) \delta x^{\mu}$, and $F_{\mu \nu}\left(x_{\mathrm{R}}+\delta x\right) \sim F\left(x_{\mathrm{R}}\right)+\partial_{\rho} F_{\mu \nu}\left(x_{\mathrm{R}}\right) \delta x^{\rho}$, so, at the bottom $\left\{i e\left(W^{-1}\left(\alpha F_{\mu \nu}+\beta \widetilde{F}_{\mu \nu}\right) W \delta x^{\mu} \delta x^{\nu}\right)\right\}_{x_{\mathrm{R}}} \approx i e\left(\alpha F_{\mu \nu}+\beta \widetilde{F}_{\mu \nu}\right) \delta x^{\mu} \delta y^{\nu}$. For the top surface we have to use these Taylor expansions for $W$ and $F_{\mu \nu}$, in the direction $\delta z^{\mu}$. This calculation is straightforward and we get $\left\{-i e\left(W^{-1}\left(\alpha F_{\mu \nu}+\beta \widetilde{F}_{\mu \nu}\right) W \delta x^{\mu} \delta x^{\nu}\right)\right\}_{x_{\mathrm{R}}+\delta z} \approx$ $-i e\left(\alpha F_{\mu \nu}+\beta \widetilde{F}_{\mu \nu}\right) \delta x^{\mu} \delta y^{\nu}-i e\left(\alpha D_{\lambda} F_{\mu \nu}+\beta D_{\lambda} \widetilde{F}_{\mu \nu}\right) \delta x^{\mu} \delta y^{\nu} \delta z^{\lambda}$. Thus, summing these contributions the terms involving the area cancel each other and then summing the three 


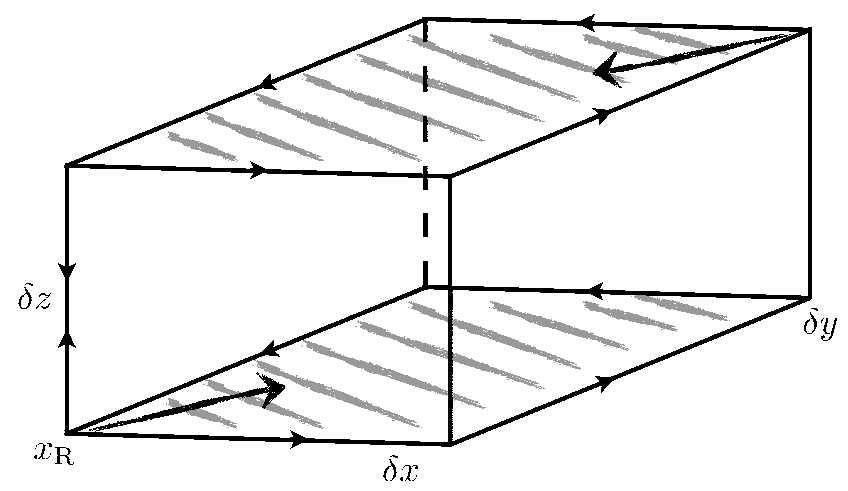

Figure 4.1 - When the volume $\Omega$ becomes the infinitesimal cube the integral equations imply the differential Yang-Mills equations. The big arrows on the bottom and top surfaces indicate the sign of $\frac{d x^{\mu}}{d \tau}$.

pairs of surfaces we get $-i e\left(\alpha D_{\lambda} F_{\mu \nu}+\beta D_{\lambda} \widetilde{F}_{\mu \nu}+\right.$ cyclic permutations $) \delta x^{\mu} \delta y^{\nu} \delta z^{\lambda}$. This is the I.h.s of Stokes theorem. The r.h.s is trivial: $\mathbb{1}+i \beta \widetilde{J}_{\mu \nu \lambda} \delta x^{\mu} \delta y^{\nu} \delta z^{\lambda}$. Clearly, the equality of the results from each side of the equation implies the differential Yang-Mills equations. Now we proceed to construct the conserved charges. Let $\Omega$ be a closed volume, so that $\partial \Omega=\emptyset$, and the integral equation (3.2.1) becomes

$$
V_{\Omega_{c}}=P_{3} \exp \left(\int_{\Omega_{c}} d \zeta d \tau V \mathcal{J} V^{-1}\right)=\mathbb{1}
$$

which express the fact that the operator $V_{\Omega_{c}}$ is independent of the paths in loop space, which in space-time are the volumes joining the infinitesimal closed surface $S_{\mathrm{R}}^{2,(0)}$ and the 2-sphere $S_{\infty}^{2,(t)}$. We now proceed to explain how to decompose $\Omega_{\mathrm{c}}$ getting the surfaces joining these 2-spheres such that the generalisation of the "spatial Wilson surface" can be obtained. Take $\Omega_{\mathrm{c}}=\Omega_{2}^{-1} \circ \Omega_{1}$, where $\Omega_{1}$ and $\Omega_{2}$ are also composed by the union of two other volumes each, as we now explain. The volume $\Omega_{1}$ links two surfaces (its borders): the infinitesimal 2-sphere around the reference point $S_{\mathrm{R}}^{2,(0)}$, and the 2-sphere at spatial infinity at time $t>0, S_{\infty}^{2,(t)}$. We then have $\Omega_{1}=\Omega_{\infty} \circ \Omega_{0}$, where $\Omega_{0}$ is the volume (or path in loop space) at $t=0$ that consists of the whole space from $S_{\mathrm{R}}^{2,(0)}$ to $S_{\infty}^{2,(0)}$, and $\Omega_{\infty}$ is the "time evolution" of the border, so it goes from $S_{\infty}^{2,(0)}$ to $S_{\infty}^{2,(t)}$. The volume $\Omega_{2}$ is made of $\Omega_{t} \circ \Omega_{-\infty}$, where $\Omega_{-\infty}$ is the "time evolution" of the border $S_{0}^{2,(0)}$, the 2-sphere around the reference point, from $t=0$ to $t>0$, where we call it $S_{\mathrm{R}}^{2,(t)}$, and $\Omega_{t}$ stands for the volume linking $S_{0}^{2,(t)}$ to the border $S_{\infty}^{2,(t)}$. The Wilson volume $V_{\Omega}$ is calculated on each of these volumes from (2.2.4). Using the decomposition explained above equation (4.1.2) is written as

$$
V_{\Omega_{-\infty}}^{-1} \cdot V_{\Omega_{t}}^{-1} \cdot V_{\Omega_{\infty}} \cdot V_{\Omega_{0}}=\mathbb{1}
$$


and now our first task is to find appropriate boundary conditions that make this an iso-spectral evolution equation for the "spatial Wilson volume" $V_{\Omega_{t}}$. Although it must be clear by now, we remember that as in the previously discussed cases the idea is to find conditions over the "connection" ( $C_{\mu}$ in 2-dimensions, $\mathcal{C}$ in 3-dimensions and $\mathcal{K}$ now) such that the Wilson operators related to the boundaries can be made equal. Here we must analyse $V_{\Omega_{ \pm \infty}}$. The "connection" appearing in (2.2.4) is $\mathcal{K}=\int_{0}^{2 \pi} d \tau V \mathcal{J} V^{-1}$, and it is calculated on the surfaces scanning the volumes. The surfaces for both borders, i.e., around the reference point and at spatial infinity, can be described topologically as cylinders; the first being $S_{\mathrm{R}}^{2} \times \mathbb{R}$ while the second $S_{\infty}^{2} \times \mathbb{R}$. In order to scan the first surface one starts at the reference point and go around the sphere $S_{\mathrm{R}}^{2}$, with a set of loops, until scan it all. Then, move forward in time to the next point in $\mathbb{R}$, and scan the sphere again, so that when it is done the opposite vertical path in $\mathbb{R}$ is taken to come back to the reference point. This goes on until a certain time $t$. Since the tiny sphere in the first surface has an infinitesimal radius it gives no contribution to $\mathcal{K}$. In fact we could split this quantity into $\mathcal{K}=\mathcal{K}_{\uparrow}+\mathcal{K}_{\circ}+\mathcal{K}_{\downarrow}$, where $\mathcal{K}_{\uparrow}$ and $\mathcal{K}_{\downarrow}$ correspond to the integrations in $\tau$ when we go up and down along $\mathbb{R}$, while $\mathcal{K}_{\circ}$ is the "connection" calculated on the sphere. Thus because of the tiny radius of the sphere $\mathcal{K}_{\circ}=0$ and we get $\mathcal{K}=\mathcal{K}_{\uparrow}+\mathcal{K}_{\downarrow}$. Since the sign of $\frac{d x^{\mu}}{d \tau}$ in $\mathcal{K}_{\uparrow}$ is the opposite of that in $\mathcal{K}_{\downarrow}$, but the integrands are the same, then $\mathcal{K}$ vanishes and the Wilson volume is the integration constant $V_{\mathrm{R}} \in Z(G)$. Now for the other border we scan in the same way, except that now the sphere is huge, with an infinite radius. It is not difficult to see that the same things happen for $\mathcal{K}_{\uparrow}$ and $\mathcal{K}_{\downarrow}$. It is now our job to find the appropriate boundary conditions to get $\mathcal{K}_{0}=0$ so that the Wilson volumes on the borders become the same. Indeed, keeping in mind that $\mathcal{K}_{\circ}$ is calculated on the sphere at spatial infinity, if we assume that at spatial infinity $J_{\mu} \sim \frac{1}{R^{2+\delta}} \quad$ and $\quad F_{\mu \nu} \sim \frac{1}{R^{\frac{3}{2}+\delta^{\prime}}}$ with $\delta, \delta^{\prime}>0$ one gets exactly the desired behaviour for $\mathcal{K}_{\circ}$ and $V_{S_{\mathbb{R}}^{2} \times \mathbb{R}}=V_{S_{\infty}^{2} \times \mathbb{R}}$, so $V_{\Omega_{\infty}^{(t)}}=V_{S_{\infty}^{2} \times \mathbb{R}} \cdot V_{\Omega_{\infty}^{(0)}} \cdot V_{S_{R}^{2} \times \mathbb{R}}^{-1}$.

As before we have to find a way to calculate the "spatial Wilson volume" at each slice of time independently; in the above equation everything is calculated from the reference point at time $t=0$. Following the same argumentations already given it is not very hard to see that $\mathcal{K}$ at the reference point at $t>0$ is related to that at $t=0$ by $\mathcal{K}_{x_{\mathrm{R}}^{t}}=W\left(x_{\mathrm{R}}^{t}, x_{\mathrm{R}}\right) \mathcal{K}_{x_{\mathrm{R}}} W^{-1}\left(x_{\mathrm{R}}^{t}, x_{\mathrm{R}}\right)$, and therefore the Wilson volume satisfies an identical relation: $V_{\Omega_{\infty}^{(t)}}^{t}=W\left(x_{\mathrm{R}}^{t}, x_{\mathrm{R}}\right) \cdot V_{\Omega_{\infty}^{(t)}}$. $W^{-1}\left(x_{\mathrm{R}}^{t}, x_{\mathrm{R}}\right)$. Finally we find the desired iso-spectral evolution

$$
V_{\Omega_{\infty}^{(t)}}^{t}=U(t) \cdot V_{\Omega_{\infty}^{(0)}} \cdot U^{-1}(t)
$$

with $U(t)=W\left(x_{\mathrm{R}}^{t}, x_{\mathrm{R}}\right) \cdot V_{S_{\mathrm{R}}^{2} \times \mathbb{R}}$. So, we have found that the eigenvalues of the operator

$$
Q \equiv P_{2} e^{i e \int_{\partial S} W^{-1}\left(\alpha F_{\mu \nu}+\beta \widetilde{F}_{\mu \nu}\right) W d x^{\mu} d x^{\nu}}=P_{3} e^{\int_{S} d \zeta d \tau V \mathcal{J} V^{-1}},
$$


$S$ being the spatial surface at a given time slice, are the conserved charges, satisfying all good properties discussed before: they are invariant under gauge transformations, re-parametrisation and change of the reference point.

Once the volume independence equation (4.1.2) is obtained we proceed to split the closed volume $\Omega_{\mathrm{c}}$ into two volumes, sharing the same boundaries. Basically, we look at that in loop space as two paths that can be deformed into each other linking two fixed points, $S_{\mathrm{R}}^{2,(0)}$ and $S_{\infty}^{2,(t)}$. That these points are fixed means that not just the physical surfaces remain the same but also if one changes their parametrisation, nothing will happen in the loop space. The scanning of $S_{\mathrm{R}}^{2,(0)}$ is trivial, so there is nothing to worry about. For $S_{\infty}^{2,(t)}$, we must analyse what happens with the Wilson surface, defined by (2.2.1). Equation (2.2.3) gives how it changes under a general variation of the space-time points. A re-parametrisation consists of variations that are parallel to the surface, then the first term, involving the 3-form with components ( $D_{\lambda} B_{\mu \nu}+$ cyclic parmutations) on a 2-dimensional surface vanishes. In order to guarantee the re-parametrisation independence one needs

$$
\begin{aligned}
& \int_{0}^{2 \pi} d \sigma \int_{0}^{\sigma} d \sigma^{\prime}\left[(\alpha-1) F_{\kappa \lambda}^{W}\left(\sigma^{\prime}\right)+\beta \widetilde{F}_{\kappa \lambda}^{W}\left(\sigma^{\prime}\right),\left(\alpha F_{\mu \nu}^{W}(\sigma)+\beta \widetilde{F}_{\mu \nu}^{W}(\sigma)\right)\right] \times \\
& \times \frac{\partial x^{\kappa}}{\partial \sigma^{\prime}} \frac{\partial x^{\mu}}{\partial \sigma}\left(\frac{\partial x^{\lambda}}{\partial \tau}\left(\sigma^{\prime}\right) \delta x^{\nu}(\sigma)-\delta x^{\lambda}\left(\sigma^{\prime}\right) \frac{\partial x^{\nu}}{\partial \tau}(\sigma)\right)=0 .
\end{aligned}
$$

This integration is performed over a loop at spatial infinity, so that the accomplishment of this condition is determined by the behaviour of the field strength ar $r \rightarrow \infty$. There are at least two sufficient conditions that make it possible to get re-parametrisation invariance: $(i)$ for $r \rightarrow \infty$ the field strength behaves like $F_{\mu \nu} \rightarrow \frac{1}{r^{2}}$ and $(i i)$ the commutator appearing above vanishes, i.e., the quantities $F_{\mu \nu}^{W}$ lie in an Abelian sub-algebra. As we discuss later, the first condition is exactly the case of instantons solutions while monopoles dyons and merons fulfil the second condition.

An analogous argumentation holds for the the charges in 3-dimensional theories. However, there it is trivial since the boundary is a circle $S_{\infty}^{1}$ and the re-parametrisations (variations tangent to the circle) imply an overall factor multiplying the equation (2.1.1), which changes nothing.

\subsection{The self-dual sector}

We are interested here in the Euclidean space-time where the Yang-Mills field strength satisfies the so called self-duality equation $F_{\mu \nu}=\kappa \widetilde{F}_{\mu \nu}$, with $\kappa= \pm 1$. We now show that using the standard non-Abelian Stokes theorem (2.1.8) we formulate the integral equation 
of self-dual Yang-Mills. Let $C_{\mu}=i e A_{\mu}$, where $A_{\mu}$ is the Yang-Mills field. Consider the quantity $H_{\mu \nu}=i e\left(\alpha F_{\mu \nu}+\kappa(1-\alpha) \widetilde{F}_{\mu \nu}\right)$, with $\alpha$ an arbitrary parameter. If one writes it as $H_{\mu \nu}=i e\left(\alpha\left(F_{\mu \nu}-\kappa \widetilde{F}_{\mu \nu}\right)+\kappa \widetilde{F}_{\mu \nu}\right)$ then it becomes evident that when the self-duality equation holds, $H_{\mu \nu}=i e F_{\mu \nu}$, coinciding with $G_{\mu \nu}=i e F_{\mu \nu}$, the curvature of $C_{\mu}$ given above. This also happens trivially in the case $\alpha=1$, when we get just an identity. The integral self-dual Yang-Mills equation is (the integration constants are in the centre of the group)

$$
P_{1} \exp \left(-i e \oint_{\partial \Sigma} A_{\mu} d x^{\mu}\right)=P_{2} \exp \left(\int_{\Sigma} W^{-1}\left(\alpha F_{\mu \nu}+\kappa(1-\alpha) \widetilde{F}_{\mu \nu}\right) W d x^{\mu} d x^{\nu}\right)
$$

for when $\Sigma$ is an infinitesimal rectangle with sides $\delta x, \delta y$ one gets (the calculations are done identically to those we discussed before) $P_{1} \exp \left(-i e \oint_{\partial \Sigma} A_{\mu} d x^{\mu}\right) \approx 1+i e F_{\mu \nu} \delta x^{\mu} \delta y^{\nu}$ and $P_{2} \exp \left(\int_{\Sigma} W^{-1}\left(\alpha F_{\mu \nu}+\kappa(1-\alpha) \widetilde{F}_{\mu \nu}\right) W d x^{\mu} d x^{\nu}\right) \approx \mathbb{1}+i e\left(\alpha F_{\mu \nu}+\kappa(1-\alpha) \widetilde{F}_{\mu \nu}\right)$, which implies the differential equations, when $\alpha=1$ is excluded. The 2-dimensional surface $\Sigma$ is a sub-manifold of the 4-dimensional Euclidean space-time $M$, and every calculation here is identical to what was done in the Chern-Simons or 3-dimensional Yang-Mills case. Also, the construction of the conserved charges is similar. The surface independence equation reads $V_{\Sigma_{\mathrm{c}}}=P_{2} e^{\int_{D_{\infty}^{(t)}} W^{-1}\left(\alpha F_{\mu \nu}+\kappa(1-\alpha) \widetilde{F}_{\mu \nu}\right) W d x^{\mu} d x^{\nu}}=\mathbb{1}$, obtained from the integral equation (4.2.1) when $\Sigma$ is closed. Now the boundary condition is $F_{\mu \nu}=\kappa \widetilde{F}_{\mu \nu} \sim \frac{1}{r^{2+\delta}}$ for $r \rightarrow \infty$. Finally the charge operator reads

$$
V_{D_{\infty}^{(t)}}^{t}=P_{2} e^{\int_{D_{\infty}^{(t)}} W^{-1}\left(\alpha F_{\mu \nu}+\kappa(1-\alpha) \widetilde{F}_{\mu \nu}\right) W d x^{\mu} d x^{\nu}}=P_{1} e^{-i e \oint_{S_{\infty}^{1,(t)}} A_{\mu} d x^{\mu}} .
$$

There is more to it. If we consider $\Sigma_{c}$ to be the boundary of a volume $\Omega$, then we can use equation (2.2.6) to get $P_{3} e^{\int_{\Omega} d \zeta d \tau V \mathcal{J} V^{-1}}=1$. This can be simplified once the self-duality equation holds, giving $P_{3} e^{i e \kappa(1-\alpha) \int_{\Omega} V \widetilde{J}_{\mu \nu \rho}^{W} V^{-1} d x^{\mu} d x^{\nu} d x^{\rho}}=1$, which is valid for any volume $\Omega$, and therefore one concludes that $J_{\mu}$ vanishes. Well, this is expected when the self-duality equations are introduced in the Yang-Mills equations.

Suppose $B_{\mu \nu}=i e \lambda F_{\mu \nu}$ in the generalised non-Abelian Stokes theorem, where $F$ is the curvature of the 1 -form Lie algebra-valued connection $A$. Then the first term of $\mathcal{K}$ in the r.h.s of the theorem vanishes, since it becomes just the Bianchi identity for $A$. What remains in

$$
P_{2} e^{i e \lambda \int_{\partial \Omega} F_{\mu \nu}^{W} d x^{\mu} d x^{\nu}}=P_{3} e^{\int_{\Omega} d \zeta d \tau V \mathcal{J} V^{-1}}
$$

is

$$
\begin{aligned}
\mathcal{J}= & e^{2} \lambda(\lambda-1) \int_{0}^{2 \pi} d \sigma \int_{0}^{\sigma} d \sigma^{\prime}\left[F_{\kappa \rho}^{W}\left(\sigma^{\prime}\right), F_{\mu \nu}^{W}(\sigma)\right] \times \\
& \times \frac{\partial x^{\kappa}}{\partial \sigma^{\prime}} \frac{\partial x^{\mu}}{\partial \sigma}\left(\frac{\partial x^{\rho}}{\partial \tau}\left(\sigma^{\prime}\right) \frac{\partial x^{\nu}}{\partial \zeta}(\sigma)-\frac{\partial x^{\rho}}{\partial \zeta}\left(\sigma^{\prime}\right) \frac{\partial x^{\nu}}{\partial \tau}(\sigma)\right),
\end{aligned}
$$


which is quite non-trivial for $\lambda \neq 0,1$; it says that the "flux" of the rescaled field strength (on the I.h.s) depends on that commutator term, and therefore is apparently non-zero even when there is no matter current. This fact deserves further investigation.

As a final remark we notice that with the self-duality condition the full Yang-Mills integral equation (4.1.1) becomes the integral Bianchi identity (4.2.3) with $\lambda=\alpha+\kappa \beta$.

\subsection{Monopoles and dyons}

We now want to evaluate the operator (4.1.4) for the monopole field given by

$$
\begin{aligned}
A_{i} & =-\frac{1}{e} \epsilon_{i j k} \frac{\hat{n}_{j}}{r} T_{k}=\frac{1}{2} \frac{i}{e} \partial_{i} g g^{-1} ; & A_{0} & =0 \\
F_{i j} & =\frac{1}{e} \epsilon_{i j k} \frac{n_{k}}{r^{2}} \hat{n} \cdot T ; & F_{0 i} & =0
\end{aligned}
$$

where $n_{i}=\frac{x_{i}}{r}$ is unit vector in the radial direction, $r^{2}=x_{1}^{2}+x_{2}^{2}+x_{3}^{2}$ defines the radial distance in the Cartesian system, $T_{i}$ are the generators of the $S U(2)$ Lie algebra satisfying

$$
\left[T_{i}, T_{j}\right]=i \epsilon_{i j k} T_{k} \quad i, j, k=1,2,3
$$

and $g$ is the group element $g=\exp (i \pi \hat{n} \cdot T)$. The field (4.3.1) corresponds to the exact Wu-Yang (36) configuration and also to the 'tHooft-Polyakov (37) configuration* at $r \rightarrow \infty$.

Lets first consider the operator (4.1.4) as the Wilson surface, defined by the surfaceordered exponential of the field strength and its dual. This quantity is then calculated on the surface of the 2-sphere at spatial infinity (and that is why our calculations hold equally for the Wu-Yang and 'tHooft-Polyakov case), by scanning it with loops based at the reference point $x_{\mathrm{R}}$. The Wilson line $W$, appearing inside the integral, is calculated from (2.1.1) integrating from the reference point to a given point on a certain loop. One then notice that the term $\hat{n} \cdot T$ appearing in the definition of the field strength is covariantly constant, i.e., $D_{i}(\hat{n} \cdot T)=$ $\partial_{i}(\hat{n} \cdot T)+i e\left[A_{i},(\hat{n} \cdot T)\right]=0$, which implies that $\frac{d}{d \sigma}\left(W^{-1} \hat{n} \cdot T W\right)=0$, and therefore $W^{-1} \hat{n} \cdot T W$ is constant along any loop, and since the sphere is covered with loops, this is constant everywhere on $S_{\infty}^{2}$. So, we can choose any point to perform the integration. Lets take it to be done at $x_{\mathrm{R}}$. The "conjugate field strength" there is then $W^{-1} F_{i j} W \equiv \frac{1}{e} \epsilon_{i j k} \frac{n_{k}}{r^{2}} T_{\mathrm{R}}$, where we denote $\hat{n} \cdot T$ at $x_{\mathrm{R}}$ by $T_{\mathrm{R}}$. Notice that since we bring everything to the reference point the surface-ordering becomes irrelevant; in fact, the "conjugate field strength" is now in the Abelian sub-algebra of $U(1)$ generated by $T_{\mathrm{R}}$. Introducing the Abelian magnetic field $B_{i}^{\mathrm{R}}=-\frac{1}{2} \epsilon_{i j k} W^{-1} F_{j k} W=-\frac{1}{e} \frac{n_{i}}{r^{2}} T_{\mathrm{R}}$ it is easy to see that the charge operator becomes the

*The Higgs field behaviour is not presented since it has no relevance for the following discussion. 
standard exponential

$$
Q=e^{-i e \alpha \int_{S_{\infty}^{2}} d \mathbf{S} \cdot \mathbf{B}^{\mathrm{R}}}=e^{i 4 \pi \alpha T_{\mathrm{R}}} .
$$

It is more convenient to define the quantity $G_{\mathrm{R}} \equiv \int_{S_{\infty}^{2}} d \mathbf{S} \cdot \mathbf{B}^{\mathrm{R}}=-\frac{4 \pi}{e} T_{\mathrm{R}}$. Thus, according to our construction, the eigenvalues of $G_{\mathrm{R}}$ are the conserved magnetic charges. Choosing a finite dimensional representation of $S U(2)$ or $S O(3)$ they are integers or half-integers. So, it follows that the magnetic charge must be quantised as integer multiples of $\frac{2 \pi}{e}$.

Now we can calculate the operator $Q$ in (4.1.4) as a volume-ordered exponential

$$
e^{-i e \alpha G_{\mathrm{R}}}=P_{3} e^{\int_{\text {space }} d \zeta d \tau V \mathcal{J}_{\text {monopole }} V^{-1}},
$$

wheret

$$
\begin{aligned}
\mathcal{J}_{\text {monopole }}= & e^{2} \alpha(\alpha-1) \int_{0}^{2 \pi} d \sigma \int_{0}^{\sigma} d \sigma^{\prime}\left[F_{\kappa \rho}^{W}\left(\sigma^{\prime}\right), F_{\mu \nu}^{W}(\sigma)\right] \times \\
& \times \frac{\partial x^{\kappa}}{\partial \sigma^{\prime}} \frac{\partial x^{\mu}}{\partial \sigma}\left(\frac{\partial x^{\rho}}{\partial \tau}\left(\sigma^{\prime}\right) \frac{\partial x^{\nu}}{\partial \zeta}(\sigma)-\frac{\partial x^{\rho}}{\partial \zeta}\left(\sigma^{\prime}\right) \frac{\partial x^{\nu}}{\partial \tau}(\sigma)\right),
\end{aligned}
$$

The 'tHooft-Poliyakov monopole has a core, and inside it the result presented before for the magnetic charge does not hold. We shall not discuss this here. We remark though that the Higgs field does not appear in our formula for the magnetic charge.

For the Wu-Yang monopole we can evaluate that integral over the entire space. In doing that we inevitably face the problem of passing through a singularity point of the gauge field. This is solved by a regularisation process of the Wilson line. For the sake of the continuation of the discussion we leave the calculations to the appendix $\mathrm{A}$ the result is that $\mathcal{J}_{\text {monopole }}$ vanishes everywhere and therefore

$$
e^{-i e \alpha G \mathrm{R}}=P_{3} e^{\int_{\text {space }} d \zeta d \tau V \mathcal{J}_{\text {monopole }} V^{-1}}=\mathbb{1} .
$$

This result implies that the magnetic charge for the Wu-Yang monopole is quantised as

$$
\text { eigenvalues of } G_{\mathrm{R}}=\frac{2 \pi n}{e \alpha} \quad n=0, \pm 1, \pm 2 \ldots
$$

If the parameter $\alpha$ is indeed arbitrary, and there is no physical condition to fix it, then the only acceptable value for the integer $n$ is $n=0$, and so the magnetic charge of the Wu-Yang monopole should vanish. Perhaps we have to go to the quantum theory to settle that issue. It might happen that quantum conditions restrict the allowed values of $\alpha$. That is one of the important points of our construction to be further investigated.

\footnotetext{
†' In the case of the 'tHooft-Poliyakov monopole, the static solution of the Higgs field requires $A_{0}=0$, which implies $J_{0}=0$, and that is why no current appears in the formula of $\mathcal{J}$ also in this case; the Wu-Yang monopole has no current by definition.
} 
Let us now consider the case of dyon solutions. For the Wu-Yang and the 'tHooft-Polyakov case, as calculated by Julia and Zee (38), the space components of the gauge potential and field tensor, namely $A_{i}$ and $F_{i j}, i, j=1,2,3$, are the same as those in (4.3.1), and the time components, at spatial infinity, are replaced by

$$
A_{0}=\frac{M}{e} \hat{n} \cdot T+\frac{\gamma}{e} \frac{\hat{n} \cdot T}{r}+O\left(\frac{1}{r^{2}}\right) ; \quad F_{0 i}=\frac{\gamma}{e} \frac{n_{i}}{r^{2}} \hat{n} \cdot T+O\left(\frac{1}{r^{3}}\right) ; \quad r \rightarrow \infty
$$

with $M$ and $\gamma$ being parameters of the solution. In the case of the $\mathrm{Wu}$-Yang dyon, i.e. when there is no Higgs field and no symmetry breaking, the formulas (4.3.8), as well as (4.3.1), are true everywhere and not only at spatial infinity. In other words, there are no terms of order $r^{-2}$ and $r^{-3}$ in $A_{0}$ and $F_{0 i}$ respectively. Concerning the quantities entering in the charge operator here we have

$$
W^{-1} \widetilde{F}_{i j} W \rightarrow-\frac{\gamma}{e} \varepsilon_{i j k} \frac{n_{k}}{r^{2}} T_{\mathrm{R}} \quad r \rightarrow \infty
$$

So, $W^{-1} \widetilde{F}_{i j} W$ also belongs to the abelian subalgebra $U(1)$ generated by $T_{\mathrm{R}}$, and it is in fact proportional to $W^{-1} F_{i j} W$. Therefore, the surface ordering is not relevant in the evaluation of the operator (4.1.4), and we get in the dyon case that

$$
Q_{S}=e^{-i e\left[\alpha \int_{S_{\infty}^{2}} d \vec{\Sigma} \cdot \vec{B}^{\mathrm{R}}+\beta \int_{S_{\infty}^{2}} d \vec{\Sigma} \cdot \vec{E}^{\mathrm{R}}\right]}=e^{-i e\left[\alpha G_{\mathrm{R}}+\beta K_{\mathrm{R}}\right]}=e^{i 4 \pi[\alpha-\beta \gamma] T_{\mathrm{R}}}
$$

where we have introduced the abelian electric field $E_{i}^{\mathrm{R}}=W^{-1} F_{0 i} W=\frac{\gamma}{e} \frac{n_{i}}{r^{2}} T_{\mathrm{R}}, \vec{B}^{\mathrm{R}}$ and $G_{\mathrm{R}}$ are the same as before, and using Gauss law we have defined

$$
\int_{S_{\infty}^{2}} d \vec{\Sigma} \cdot \vec{E}^{R}=K_{\mathrm{R}} \quad \text { and so } \quad K_{\mathrm{R}}=\frac{4 \pi \gamma}{e} T_{\mathrm{R}}
$$

According to (4.1.4) the eigenvalues of $Q_{S}$ are constant in time, and so we conclude from (4.3.10) that the eigenvalues of $\left(\alpha G_{\mathrm{R}}+\beta K_{\mathrm{R}}\right)$ are constants. But if we assume that the parameters $\alpha$ and $\beta$ are arbitrary it follows that the eigenvalues of $G_{\mathrm{R}}$ and $K_{\mathrm{R}}$ are independently constant in time. We have seen that, by evaluating the eigenvalues of $T_{\mathrm{R}}$ on finite dimensional representations of the gauge group $S U(2)$ or $S O(3)$, where they are integers or half-integers, the magnetic charges, eigenvalues of $G_{\mathrm{R}}$, are quantised. Under the same assumptions it follows that

$$
\text { eigenvalues of } K_{\mathrm{R}}=\frac{2 \pi \gamma n}{e} \quad n=0, \pm 1, \pm 2, \ldots
$$

Again from (4.1.4) we can express the charges in terms of volume ordered integrals:

$$
e^{-i e\left[\alpha G_{\mathrm{R}}+\beta K_{\mathrm{R}}\right]}=P_{3} e^{\int_{\text {space }} d \zeta d \tau V \mathcal{J}_{\text {dyon }} V^{-1}}
$$


with

$$
\begin{aligned}
\mathcal{J}_{\text {dyon }} \equiv & \int_{0}^{2 \pi} d \sigma\left\{i e \beta \widetilde{J}_{i j k}^{W} \frac{d x^{i}}{d \sigma} \frac{d x^{j}}{d \tau} \frac{d x^{k}}{d \zeta}\right. \\
+ & e^{2} \int_{0}^{\sigma} d \sigma^{\prime}\left[\left((\alpha-1) F_{i j}^{W}+\beta \widetilde{F}_{i j}^{W}\right)\left(\sigma^{\prime}\right),\left(\alpha F_{k l}^{W}+\beta \widetilde{F}_{k l}^{W}\right)(\sigma)\right] \\
& \left.\times \frac{d x^{i}}{d \sigma^{\prime}} \frac{d x^{k}}{d \sigma}\left(\frac{d x^{j}\left(\sigma^{\prime}\right)}{d \tau} \frac{d x^{l}(\sigma)}{d \zeta}-\frac{d x^{j}\left(\sigma^{\prime}\right)}{d \zeta} \frac{d x^{l}(\sigma)}{d \tau}\right)\right\}
\end{aligned}
$$

In the case of the Wu-Yang dyon we have $\widetilde{J}_{123}=J_{0}=0$, since there are no sources. However, for the Julia-Zee dyon we have that the Higgs field contributes to the current $J_{\mu}$. One can extract the operators $G_{\mathrm{R}}$ and $K_{\mathrm{R}}$ from (4.3.13), by setting $\beta=0$ and $\alpha=0$ respectively. Then, the Higgs field contributes to the electric charge only.

In the case of the $\mathrm{Wu}$-Yang dyon it is possible to evaluate (4.3.14) after a regularisation of the Wilson line operator passing through the singularity of the gauge potential (4.3.1). That calculation is shown in the appendix $\mathrm{A}$ and it was found that $\mathcal{J}_{\text {dyon }}$ vanishes in all loops for the Wu-Yang dyon. Therefore

$$
e^{-i e\left[\alpha G_{\mathrm{R}}+\beta K_{\mathrm{R}}\right]}=P_{3} e^{\int_{\text {space }} d \zeta d \tau V \mathcal{J}_{\text {dyon }} V^{-1}}=\mathbb{1}
$$

which implies that

$$
\text { eigenvalues of }\left[\alpha G_{\mathrm{R}}+\beta K_{\mathrm{R}}\right]=\frac{2 \pi n}{e} \quad n=0, \pm 1, \pm 2, \ldots
$$

Again, if the parameters $\alpha$ and $\beta$ are indeed arbitrary, then it follows from (4.3.16) that by taking $\beta=0$, the eigenvalues of $G_{\mathrm{R}}$ should obey (4.3.7). On the other hand by taking $\alpha=0$, one concludes that (4.3.16) implies that

$$
\text { eigenvalues of } K_{\mathrm{R}}=\frac{2 \pi n}{e \beta} \quad n=0, \pm 1, \pm 2 \ldots
$$

Now, if (4.3.7) and (4.3.17) should hold true for arbitrary values of $\alpha$ and $\beta$ respectively, then the only acceptable value of the integer $n$ in both equations is $n=0$, and consequently the electric and magnetic charges of the Wu-Yang dyon should vanish. As discussed below (4.3.7), we have perhaps to consider of the quantum theory to settle that issue, since there could be quantum conditions restricting the values of $\alpha$ and $\beta$.

Suppose now that we consider the standard non-Abelian Stokes theorem (2.1.8) where $\Sigma$ is a closed surface, boundary of the volume $\Omega$. Then, we have the identity (with $C_{\mu}=i e A_{\mu}$ )

$$
P_{2} e^{i e \int_{\partial \Omega} W^{-1} F_{\mu \nu} W d x^{\mu} d x^{\nu}}=1
$$


For any configuration satisfying $F_{\mu \nu} \sim \frac{1}{r^{2+\delta}}$, with $\delta>0$ at $r \rightarrow \infty$ one can build up, using this identity, the iso-spectral evolution of the operator

$$
V_{x_{\mathrm{R}}^{(t)}}^{\prime}\left(D_{\infty}^{(t)}\right)=P_{2} e^{i e \int_{D_{\infty}^{(t)}} d \sigma d \tau W^{-1} F_{\mu \nu} W \frac{d x^{\mu}}{d \sigma} \frac{d x^{\nu}}{d \tau}}=P_{1} e^{-i e \oint_{S_{\infty}^{1,(t)}} d \sigma A_{\mu} \frac{d x^{\mu}}{d \sigma}}
$$

Note that if $D_{\infty}^{(t)}$ is a spatial surface then the surface ordered integral in (4.3.18), namely $P_{2} e^{i e \int_{D_{\infty}^{(t)}} d \sigma d \tau W^{-1} F_{\mu \nu} W \frac{d x^{\mu}}{d \sigma} \frac{d x^{\nu}}{d \tau}}$, corresponds to the flux of the non-abelian magnetic field $\left(B_{i} \equiv\right.$ $\left.-\frac{1}{2} \varepsilon_{i j k} F_{j k}\right)$ through that surface. On the other hand, if $D_{\infty}^{(t)}$ has a time component then that integral corresponds to the flux of the non-abelian electric field $\left(E_{i} \equiv F_{0 i}\right)$ through such spatialtemporal surface. Note that the conservation of those fluxes can be intuitively understood by the fact that the border of $D_{\infty}^{(t)}$ is the circle $S_{\infty}^{1,(t)}$ of infinite radius. Therefore, if the field configuration is localized in a region at a finite distance to the plane containing $S_{\infty}^{1,(t)}$ the solid angle defined by that circle is $2 \pi$ spheroradians. If that field configuration evolves in the time $t$ changing its distance to that plane by a finite amount, the solid angle will remain the same, and so should the flux of the magnetic or electric fields. Of course, that is an intuitive view, and so not precise, of the conservation of the charge, but we will show that it stands reasonable in the examples we discuss here.

It is worth evaluating the conserved charges associated to the operator (4.3.18) in the case of the monopole and dyon solutions. For simplicity we shall take the circle of infinite radius $S_{\infty}^{1,(t)}$ to lie on the plane $x^{1} x^{2}$, for some constant values of $x^{3}$ and $x^{0}$. The calculation for any other plane is similar and leads, as we shall see, to similar results. We use polar coordinates on the plane, with the polar angle being the parameter $\sigma$ parametrising the circle, i.e. $x^{1}=\rho \cos \sigma, x^{2}=\rho \sin \sigma$, and $r^{2}=\rho^{2}+\left(x^{3}\right)^{2}$, with $x^{3}$ constant, and $\rho \rightarrow \infty$. Therefore, for both the monopole and dyon solutions, we get from (4.3.1) that on the circle of infinite radius we have $A_{\mu} \frac{d x^{\mu}}{d \sigma}=\frac{1}{e} T_{3}$, since on that circle $\rho \sim r \rightarrow \infty$ and the unit vector $\hat{n}$, on that limit, has components only on the plane $x^{1} x^{2}$. Therefore, from (4.3.18), we have

$$
V_{x_{\mathrm{R}}^{(t)}}^{\prime}\left(D_{\infty}^{(t)}\right)=P_{1} e^{-i e \oint_{S_{\infty}^{1,(t)}} d \sigma A_{\mu} \frac{d x^{\mu}}{d \sigma}}=e^{-i 2 \pi T_{3}}
$$

The eigenvalues of (4.3.18) are conserved in the time $t$ which in this case can be any linear combination of $x^{0}$ and $x^{3}$. But that is equivalent to say that the eigenvalues of $T_{3}$ are conserved in $t$. Since those are integers or half integers in a finite dimensional representation of $S U(2)$, it follows that the operator $V_{x_{R}^{\prime(t)}}^{\prime}\left(\mathcal{D}_{\infty}^{(t)}\right)$ is either $\mathbb{1}$ or $-\mathbb{1}$, i.e. an element of the centre of $S U(2)$. As pointed out below (4.3.18) such conserved charge can be interpreted as the non-abelian magnetic flux through the surface $D_{\infty}^{(t)}$, which border is $S_{\infty}^{1,(t)}$. Indeed, we see that the argument of the exponential in (4.3.19) is half of the argument of the exponential 
in (4.3.3), if one takes $\alpha=1$, and considers that $T_{\mathrm{R}}$ and $T_{3}$ have the same norm and so the same eigenvalues. Remember $T_{\mathrm{R}}$ is the value of $\hat{n} \cdot T$ at the reference point $x_{\mathrm{R}}$ and so $\operatorname{Tr}\left(T_{\mathrm{R}}\right)^{2}=\operatorname{Tr}\left(T_{i} T_{j}\right) n_{i} n_{j}=\lambda$, where $\operatorname{Tr}\left(T_{i} T_{j}\right)=\lambda \delta_{i j}$, and $\lambda$ depends upon the representation used. Since the argument of the exponential in (4.3.3) corresponds to the total flux of the magnetic field through $S_{\infty}^{2}$, we see that it is the double of the flux through $D_{\infty}^{(t)}$. Due to the spherical symmetry of the solution that is compatible with interpretation, given below (4.3.18), since $S_{\infty}^{2}$ corresponds to a solid angle of $4 \pi$ spheroidal as seen from the centre of the solution and $\mathcal{D}_{\infty}^{(t)}$ corresponds to only $2 \pi$ steradians.

There are several important comments regarding our charges for monopoles and dyons. First of all we recall that usually the charges associated to the Yang-Mills theory are defined from the current $j^{\mu}=\partial_{\nu} F^{\nu \mu}$ and its dual, and with appropriate boundary conditions are writes as

$$
Q_{\mathrm{YM}}=\int_{S_{\infty}^{2}} d \mathbf{S} \cdot \mathbf{E} \quad \widetilde{Q}_{\mathrm{YM}}=\int_{S_{\infty}^{2}} d \mathbf{S} \cdot \mathbf{B}
$$

where $E_{i}=F_{0 i}$ and $B_{i}=-\frac{1}{2} \epsilon_{i j k} F_{j k}$ are the non-Abelian electric and magnetic fields.

The charges we constructed are different from those given by (4.3.20). Indeed, from (4.3.1) and (4.3.8) we have that the magnetic and electric fields at spatial infinity for the Wu-Yang and 'tHooft-Polyakov cases are given by

$$
B_{i} \rightarrow-\frac{1}{e} \frac{n_{i}}{r^{2}} \hat{n} \cdot T ; \quad E_{i} \rightarrow \frac{\gamma}{e} \frac{n_{i}}{r^{2}} \hat{n} \cdot T ; \quad r \rightarrow \infty
$$

So, they do not lie on an abelian $U(1)$ subalgebra like $B_{i}^{\mathrm{R}}$ and $E_{i}^{\mathrm{R}}$ given above, and when integrated on the two-sphere at infinity lead to the vanishing of the charges (4.3.20), i.e.

$$
Q_{Y M}^{\text {monopole/dyon }}=\widetilde{Q}_{Y M}^{\text {monopole/dyon }}=0
$$

Note that even though the evaluation of the charges (4.3.3) and (4.3.10) rely on the choice of a reference point $x_{R}$, which leads to a particular generator $T_{R}$, the charges do not depend upon that reference point. Indeed, if one changes the reference point from $x_{\mathrm{R}}$ to $\widetilde{x}_{\mathrm{R}}$, then the operator $Q_{S}$ changes as $Q_{S} \rightarrow W^{-1}\left(\widetilde{x}_{\mathrm{R}}, x_{\mathrm{R}}\right) \cdot Q_{S} \cdot W\left(\widetilde{x}_{\mathrm{R}}, x_{\mathrm{R}}\right)$ where $W\left(\widetilde{x}_{\mathrm{R}}, x_{\mathrm{R}}\right)$ is the holonomy from the old reference point $x_{\mathrm{R}}$ to the new one $\widetilde{x}_{\mathrm{R}}$. Therefore the charges, which are the eigenvalues of $Q_{S}$, do not change.

Also, under gauge transformations $g \in G$ the charges defined by (4.3.20) become

$$
Q_{\mathrm{YM}} \rightarrow \int_{S_{\infty}^{2}} d \mathbf{S} \cdot g \mathbf{E} g^{-1} \quad \widetilde{Q}_{\mathrm{YM}} \rightarrow \int_{S_{\infty}^{2}} d \mathbf{S} \cdot g \mathbf{B} g^{-1},
$$

and the eigenvalues of them remain invariant only under gauge transformations that go to a 
constant $g_{\infty}$ at infinity: $Q_{\mathrm{YM}} \rightarrow g_{\infty} Q_{\mathrm{YM}} g_{\infty}^{-1}, \widetilde{Q}_{\mathrm{YM}} \rightarrow g_{\infty} \widetilde{Q}_{\mathrm{YM}} g_{\infty}^{-1}$. The charges we constructed here are invariant under general gauge transformations instead, and not just to these restricted ones.

Note that since the charges are the eigenvalues of the operator (4.1.4), the number of charges is equal to the rank of the gauge group $G$. However, since the field tensor and its Hodge dual come multiplied by the arbitrary parameters $\alpha$ and $\beta$ respectively, the number of charges is in fact twice the rank of the gauge group. So, we have rank of $G$ magnetic charges and rank of $G$ electric charges. In this sense the number of charges does not pay attention to the pattern of symmetry breaking. Indeed, our calculations have shown that the electric and magnetic charges are the same for the Wu-Yang case, which is a solution of the pure Yang-Mills theory, and for the 'tHooft-Polyakov case which has a Higgs field breaking the gauge symmetry from $S O(3)$ down to $S O(2)$. In fact, as we have shown above the Higgs field does not play any role in the evaluation of the charges. In addition the conservation of the charges is dynamical, i.e. it follows directly from the integral form of the equations of motion (4.1.1). That contrasts to the conservation of the magnetic charge of the 'tHooftPolyakov monopole which follows from topological (homotopy) considerations related to the mapping of the Higgs field from the spatial infinity to the Higgs vacua. Another point relates to the quantisation of the magnetic charge, which in the case of 'tHooft-Polyakov monopole comes from the topology again, i.e. the charge is determined by second homotopy group of the Higgs vacua. In our case, the quantization of the charges comes from the integral equations of motion themselves, without any reference to the Higgs field since it works equally well for the Wu-Yang and 'tHooft-Polyakov monopoles. It is worth pointing out that the magnetic charges of monopoles of 'tHooft-Polyakov type have already been expressed in the literature, as surface ordered integral using the ordinary non-abelian Stokes theorem. See for instance section 5 of Goddard and Olive's review paper (39). However, that construction is totally based on the properties of the Higgs vacua, since the fact that the Higgs field must be covariantly constant at spatial infinity leads to an equation for it similar to (2.1.1) for the Wilson line $W$. In addition the argument for the conservation of the magnetic charge is particular to that type of solution since it is based on topology considerations of the solution. The generalised non-Abelian Stokes theorem (2.2.6), and consequently the integral Yang-Mills equations (4.1.1) were not known by that time. We believe that the role played by the integral Yang-Mills equation in monopole and dyon solutions deserves further study specially in the quantum theory. It might connect to the so-called Abelian projection and arguments for confinement. 


\subsection{Instantons}

The instantons are euclidean self-dual solutions where the gauge potentials become of the pure gauge form at infinity, i.e. $A_{\mu} \rightarrow \frac{1}{e} \partial_{\mu} g g^{-1}$, for $s \rightarrow \infty$, with $s^{2}=x_{1}^{2}+x_{2}^{2}+x_{3}^{2}+x_{4}^{2}$, where $x^{\mu}, \mu=1,2,3,4$, being the Cartesian coordinates in the Euclidean space-time.

Let us consider the case where the gauge group is $S U(2)$ and take the one-instanton solution (4) given by

$$
A_{\mu}=-\frac{2}{e} \sigma_{\mu \nu} \frac{x^{\nu}-a^{\nu}}{\left(x^{\rho}-a^{\rho}\right)^{2}+\lambda^{2}} ; \quad F_{\mu \nu}=\kappa \widetilde{F}_{\mu \nu}=\frac{\sigma_{\mu \nu}}{e} \frac{4 \lambda^{2}}{\left[\left(x^{\rho}-a^{\rho}\right)^{2}+\lambda^{2}\right]^{2}}
$$

with $\kappa= \pm 1$, and where $a^{\mu}, \mu=1,2,3,4$, and $\lambda$ are parameters of the solution, and

$$
\sigma_{i 4}=-\kappa T_{i} ; \quad \sigma_{i j}=\varepsilon_{i j k} T_{k} ; \quad\left[T_{i}, T_{j}\right]=i \varepsilon_{i j k} T_{k}
$$

with $i, j, k=1,2,3, T_{i}$ being the generators of the $S U(2)$ Lie algebra, and the quantities $\sigma_{\mu \nu}$ satisfy $\frac{1}{2} \varepsilon_{\mu \nu \alpha \beta} \sigma_{\alpha \beta}=\kappa \sigma_{\mu \nu}$.

If one considers a 2 -sphere $S_{\infty}^{2}$ of infinite radius surrounding the instanton then we have that the integrand in the surface ordered integral in (4.1.4) behaves as

$$
(\alpha+\kappa \beta) F_{\mu \nu} \frac{\partial x^{\mu}}{\partial \sigma} \frac{\partial x^{\nu}}{\partial \tau} \rightarrow \frac{1}{r^{2}} \quad \text { as } \quad r \rightarrow \infty
$$

where $r$ is the radius of $S_{\infty}^{2}$. Therefore, the operator (4.1.4) is unity, i.e. $Q_{S_{\infty}^{2}}=1$, and that unity comes from the exponentiation of the trivial element of the Lie algebra. So, the one-instanton solution have vanishing charges associated to (4.1.4) .

Let us now evaluated the charges associated to the operator (4.2.2). Without any loss of generality take the circle $S_{\infty}^{1,(t)}$ of infinite radius to lie on the plane $x^{1} x^{2}$, at some constant values of $x^{3}$ and $x^{4}$. Due to the symmetries of the one-instanton solution the calculation on any other plane is very similar. We shall use polar coordinates $x^{1}=\rho \cos \sigma, x^{2}=\rho \sin \sigma$, with $s^{2}=\rho^{2}+\left(x^{3}\right)^{2}+\left(x^{4}\right)^{2}$, and $\rho \rightarrow \infty$, where we have taken the polar angle $\sigma$ to be the same as the parameter of the circle $S_{\infty}^{1,(t)}$. Therefore, using (4.4.1), the integrand of the path ordered integral in (4.2.2) becomes

$$
A_{\mu} \frac{d x^{\mu}}{d \sigma}=-(2 / e) \rho\left(-\sigma_{1 \nu} \sin \sigma+\sigma_{2 \nu} \cos \sigma\right) \frac{\left(x^{\nu}-a^{\nu}\right)}{\left(x^{\mu}-a^{\mu}\right)^{2}-\lambda^{2}} .
$$

As $\rho \rightarrow \infty$, the only non-vanishing terms are those where $x^{\nu}$ is one of the coordinates of the 
plane, i.e. $x^{1}$ or $x^{2}$. Then $A_{\mu} \frac{d x^{\mu}}{d \sigma} \rightarrow(2 / e) \sigma_{12}$, and so (4.2.2) becomes

$$
V_{D_{\infty}}=P_{1} e^{-i e \oint_{S_{\infty}^{1,(t)}} d \sigma A_{\mu} \frac{d x^{\mu}}{d \sigma}}=e^{-i 2 \int_{0}^{2 \pi} d \theta \sigma_{12}}=e^{-i 4 \pi T_{3}}
$$

where $D_{\infty}$ is the infinity disk with border $S_{\infty}^{1,(t)}$ on the plane $x^{1} x^{2}$. From (4.2.2) this operator should correspond to the (euclidean) magnetic flux $\Phi$ of $B_{i}=-\frac{1}{2} \epsilon_{i j k} F_{j k}$ through $D_{\infty}$, i.e. $V_{D_{\infty}}=e^{-i e \Phi\left(\mathbf{B}, D_{\infty}\right)}$. If one takes finite dimensional representations of $S U(2)$ or $S O(3)$, the eigenvalues of $T_{3}$ are integers or half integers and so $V_{D_{\infty}}=\mathbb{1}$, which is compatible with the fact the connection $A_{\mu}$ for the one-instanton is flat in the limit $s \rightarrow \infty$. However, that fact also implies that the flux should be quantised as

$$
\Phi\left(\mathbf{B}, D_{\infty}\right)=\frac{2 \pi n}{e} \quad n=0, \pm 1, \pm 2, \ldots
$$

However, following the same reasoning, the charges coming from (4.1.4) should also be associated, in such self-dual case solution, to the (euclidean) magnetic flux through the closed sphere $S_{\infty}^{2}$. But as we have shown below (4.4.3) that flux must vanish. Therefore, the only compatible value of $n$ in (4.4.6) seems to be $n=0$.

Let us now consider the case of the two-instanton solution. A closed form for the regular (non-singular) form of that solution is not easy. However we need only its asymptotic form to calculate the charges and that is provided by Giambiagi and Rothe (40). Consider a twoinstanton regular solutions where the position four-vector of each instanton is given by $a_{1}^{\mu}$ and $a_{2}^{\mu}$. Then the asymptotic form of the connection is given by (40)

$$
A_{\mu} \rightarrow \frac{4}{e a^{2} s^{2}}\left[(x \cdot a) \sigma_{\mu \lambda} b_{\lambda}+b_{\mu} x_{\nu} \sigma_{\nu \lambda} b_{\lambda}\right] \quad \text { as } \quad s \rightarrow \infty
$$

where $s^{2}=x_{1}^{2}+x_{2}^{2}+x_{3}^{2}+x_{4}^{2}, \sigma_{\mu \nu}$ is the same as in (4.4.2), $a_{\mu}$ is the difference between the two position four-vectors, and $b_{\mu}$ is the reflection of $a_{\mu}$ through the hyperplane perpendicular to $x_{\mu}$, i.e.

$$
a^{\mu} \equiv a_{1}^{\mu}-a_{2}^{\mu} \quad b_{\mu} \equiv a_{\mu}-2 \frac{(x \cdot a)}{x^{2}} x_{\mu}
$$

and so $b^{2}=a^{2}$.

The leading term of the connection in (4.4.7) is flat, falling as $1 / s$ as $s \rightarrow \infty$. The leading term of the field tensor which would fall as $1 / s^{2}$ vanishes, and therefore $F_{\mu \nu}$ falls at least as $1 / s^{3}$. Consequently, the integrand of the surface ordered integral in (4.1.4), namely $F_{\mu \nu} \frac{d x^{\mu}}{d \sigma} \frac{d x^{\nu}}{d \tau}$, falls faster than $1 / s$, so it vanishes in the limit $s \rightarrow \infty$. We then conclude that, similarly to the one-instanton case, the charges associated to the operator (4.1.4) vanish when evaluated on the two-instanton solution.

\footnotetext{
$¥$ Use the self-duality condition in the integrand on the I.h.s of (4.2.2).
} 
We now evaluate the charges associated to the operator (4.2.2) for the two-instanton solution. Given an infinite plane (disk) $\mathcal{D}_{\infty}$ with border being the circle $S_{\infty}^{1,(t)}$ of infinite radius we can choose, without loss of generality, the axis $x^{1}$ and $x^{2}$ to lie on that plane. We then split the vector $a^{\mu}$ in its perpendicular and parallel parts with respect to the plane, i.e. $a^{\mu}=a_{\perp}^{\mu}+a_{\|}^{\mu}$, and take the axis $x^{1}$ to lie along $a_{\|}^{\mu}$, and the axis $x^{3}$ to lie along $a_{\perp}^{\mu}$. In addition we take polar coordinates on the plane $x^{1} x^{2}$, such that $x^{1}=\rho \cos \sigma$, and $x^{2}=\rho \sin \sigma$, with the polar angle $\sigma$ being the same as the parameter in (4.2.2), parametrising $S_{\infty}^{1,(t)}$. Then the integrand of the path ordered integral in (4.2.2) along the infinite circle $S_{\infty}^{1,(t)}$ for the connection (44.4.7) becomes $(\rho \rightarrow \infty)$

$$
A_{\mu} \frac{d x^{\mu}}{d \sigma} \rightarrow \frac{4}{e}\left[\frac{\left|a_{\|}\right|^{2}}{a^{2}} T_{3}+\frac{\left|a_{\|}\right|\left|a_{\perp}\right|}{a^{2}}\left[\cos (2 \sigma) T_{1}+\sin (2 \sigma) T_{2}\right]\right]
$$

We now perform a gauge transformation $A_{\mu} \rightarrow\left(g_{2} g_{1}\right) A_{\mu}\left(g_{2} g_{1}\right)^{-1}+\frac{i}{e} \partial_{\mu}\left(g_{2} g_{1}\right)\left(g_{2} g_{1}\right)^{-1}$, with $g_{1}=e^{i 2 \sigma T_{3}}$ and $g_{2}=e^{i 2 \varphi T_{2}}$. The angle $\varphi$ is defined as follows: since $a^{2}=\left|a_{\|}\right|^{2}+\mid$ $\left.a_{\perp}\right|^{2}$, we parametrise it as $\left|a_{\|}\right|=|a| \cos \varphi$, and $\left|a_{\perp}\right|=|a| \sin \varphi$, with $0 \leq \varphi \leq \frac{\pi}{2}$. So, since the vector $a_{\mu}$ was chosen to lie on the plane $x^{1} x^{3}, \varphi$ is the angle between $a_{\mu}$ and the plane $x^{1} x^{2}$ measured along the plane $x^{1} x^{3}$. Under such a gauge transformation one gets that

$$
A_{\mu} \frac{d x^{\mu}}{d \sigma} \rightarrow A_{\mu}^{\prime} \frac{d x^{\mu}}{d \sigma}=\frac{2}{e} T_{3}
$$

and so

$$
P_{1} e^{-i e \oint_{S_{\infty}^{1}} d \sigma A_{\mu} \frac{d x^{\mu}}{d \sigma}} \rightarrow g_{1}(\sigma=2 \pi)^{-1} g_{2}^{-1} P_{1} e^{-i e \oint_{S_{\infty}^{1}} d \sigma A_{\mu}^{\prime} \frac{d x^{\mu}}{d \sigma}} g_{2} g_{1}(\sigma=0)
$$

Finally, the operator (4.2.2) becomes

$$
V\left(\mathcal{D}_{\infty}\right)=P_{1} e^{-i e \oint_{S_{\infty}^{1}} d \sigma A_{\mu} \frac{d x \mu}{d \sigma}}=e^{-i 4 \pi T_{3}} e^{-i 2 \varphi T_{2}} e^{-i 4 \pi T_{3}} e^{i 2 \varphi T_{2}}
$$

We can try to interpret that result in terms of the (euclidean) magnetic flux $\Phi$ through the infinite disk $D_{\infty}$. Since we are dealing with a non-abelian gauge theory one should not expect a linear superposition of the fluxes of each instanton. We have seen in (4.4.5) that the exponentiated flux of a single instanton is $e^{-i 4 \pi T_{3}}$. In addition, since $\varphi$ is the angle between the line passing through the centres of the instantons and the disc $D_{\infty}$, the result (4.4.12) could give a hint on how the fluxes compose. That is certainly a point which deserves further study. Again, as in any finite dimensional representation of $S U(2)$ we have that $e^{-i 4 \pi T_{3}}=1$, and so $V_{D_{\infty}}=1$, which is compatible with the fact that $A_{\mu}$ is flat at the leading order we have performed the calculation. Using the flux interpretation of the charges we can write $V_{D_{\infty}}=e^{-i e \Phi_{2-\text { inst. }}\left(D_{\infty}\right)}$, and so the two-instanton flux $\Phi_{2 \text {-inst. }}\left(D_{\infty}\right)$ is quantised as in (4.4.6). 


\subsection{Merons}

Merons are singular euclidean solutions not self-dual with one-half unit of topological charge (see (41-43)). We shall work here with such solutions in the Coulomb gauge since is is more suitable for the evaluation of the charges and it also connects with monopole solutions. The solution for a one-meron located at the origin is given by $(41,44)$

$$
A_{i}=-\frac{1}{e} \varepsilon_{i j k} \frac{n_{j}}{r}\left(1-\frac{x_{4}}{\sqrt{x_{4}^{2}+r^{2}}}\right) T_{k} \quad A_{4}=0
$$

with $r^{2}=x_{1}^{2}+x_{2}^{2}+x_{3}^{2}, i, j, k=1,2,3$, and $T_{k}$ are the generators of the $S U(2)$ Lie algebra. Note that for $x_{4}=0$ the connection (4.5.1) coincides with that for the Wu-Yang monopole given in (4.3.1). In addition, it interpolates between two vacuum configurations, i.e. for $x_{4} \rightarrow \infty$ the connection (4.5.1) vanishes, and for $x_{4} \rightarrow-\infty$ it becomes of a pure gauge form $A_{i}=\frac{i}{e} \partial_{i} g g^{-1}$, with $g=\exp (i \pi \hat{n} \cdot T)$.

In order to evaluate the charges (4.1.4) we need the field tensor at infinity, which is given by

$$
F_{i j} \rightarrow \frac{1}{e} \varepsilon_{i j k} \frac{n_{k}}{r^{2}} \hat{n} \cdot T \quad F_{4 i} \rightarrow \frac{1}{e} \varepsilon_{i j k} \frac{n_{j}}{r^{2}} T_{k} \quad r \rightarrow \infty
$$

Note that when taking the limit $r \rightarrow \infty$ we have kept $x_{4}$ finite. The double limit $r \rightarrow \infty$ and $x_{4} \rightarrow \pm \infty$, is not well defined. The asymptotic form of the space components of the dual tensor is $\left(\varepsilon_{1234}=1\right)$

$$
\widetilde{F}_{i j} \rightarrow-\frac{1}{e} \frac{1}{r^{2}}\left[n_{i} T_{j}-n_{j} T_{i}\right] \quad r \rightarrow \infty .
$$

If we evaluate the operator (4.1.4) on a spatial 2-sphere $S_{\infty}^{2}$ of infinite radius and centred at the origin, it turns out that $\hat{n}$ is perpendicular to $S_{\infty}^{2}$ and the derivatives $\frac{d x^{i}}{d \sigma}$ and $\frac{d x^{i}}{d \tau}$, with $\sigma$ parametrising the loops scanning the sphere and $\tau$ labelling them, are parallel to $S_{\infty}^{2}$. Therefore, we have that $\widetilde{F}_{i j} \frac{\partial x^{i}}{\partial \sigma} \frac{\partial x^{j}}{\partial \tau}=0$. Consequently, the calculation of the operator (4.1.4) for the one-meron solution is identical to that for the monopole (see calculation leading to (4.3.3)). So, we have that

$$
Q_{S}=P_{2} e^{i e \int_{S_{\infty}^{2}} d \sigma d \tau W^{-1}\left[\alpha F_{i j}+\beta \widetilde{F}_{i j}\right] W \frac{\partial x^{i}}{\partial \sigma} \frac{\partial x^{j}}{\partial \tau}}=e^{-i e \alpha \int_{S_{\infty}^{2}} d \vec{\Sigma} \cdot \vec{B}^{\mathrm{R}}}=e^{-i e \alpha G_{\mathrm{R}}}=e^{i 4 \pi \alpha T_{\mathrm{R}}}(4.5
$$

where we have introduced a (euclidean) magnetic field in a way similar to that in (4.3.3), i.e. $B_{i}^{\mathrm{R}} \equiv-\frac{1}{2} \epsilon_{i j k} W^{-1} F_{j k} W=-\frac{1}{e} \frac{n_{i}}{r^{2}} T_{\mathrm{R}}$, with $T_{\mathrm{R}}$ being the value of $\hat{n} \cdot T$ at the reference point $x_{\mathrm{R}}$ used in the scanning of the sphere. Using the same arguments as in the case of the monopole we conclude that the magnetic charges, eigenvalues of $G_{\mathrm{R}}$ are quantised as in 
(4.3.7).

We then conclude that the one-meron solution has a magnetic charge conserved in the euclidean time $x_{4}$, and it is quantised in units of $\frac{2 \pi}{e}$. What it is not clear is what happens to that charge in the limit $x_{4} \rightarrow \pm \infty$, since as we have seen, the connection (4.5.1) becomes flat in that limit, and so the charge should disappear. One of the difficulties in answering that is the fact that the double limit $r \rightarrow \infty$ and $x_{4} \rightarrow \pm \infty$, of the connection (4.5.1) is not well defined.

The evaluation of the charges from the operator (4.3.18) for the one-meron solution is also identical to that of the monopole. Indeed, if we consider the circle $S_{\infty}^{1,(t)}$ of infinite radius to lie on spatial planes, then only the components $A_{i}, i=1,2,3$, of the connection matters. But in the limit $r \rightarrow \infty$ the connection (4.5.1) becomes identical to that for the monopole (4.3.1). Therefore one gets that

$$
V_{x_{\mathrm{R}}^{(t)}}^{\prime}\left(\mathcal{D}_{\infty}^{(t)}\right)=P_{1} e^{-i e \oint_{S_{\infty}^{1,(t)}} d \sigma A_{i} \frac{d x^{i}}{d \sigma}}=e^{-i 2 \pi T_{3}}
$$

The eigenvalues of that operator are conserved in the euclidean time $x_{4}$, and their interpretation, given below (4.3.19), in terms of the magnetic flux through the surface which border is $S_{\infty}^{1,(t)}$ remains valid. Again, we do not know what happens to those charges in the limit $x_{4} \rightarrow \pm \infty$, for the same reasons given above in the case of the one-meron magnetic charge.

The two-meron solution in the Coulomb gauge corresponding to one meron siting at the position $x^{\mu}=a^{\mu}=(0,0,0, a)$ and the other at $x^{\mu}=b^{\mu}=(0,0,0, b)$ is given by $(41,44)$

$$
A_{i}=-\frac{1}{e} \epsilon_{i j k} \frac{n_{j}}{r}\left(1+\frac{r^{2}+\left(x_{4}-a\right)\left(x_{4}-b\right)}{\sqrt{(x-a)^{2}(x-b)^{2}}}\right) T_{k} \quad A_{4}=0
$$

Expanding it in powers of $\frac{1}{r}$ one gets

$$
A_{i}=\frac{i}{e} \partial_{i} g g^{-1}+\frac{1}{e} \frac{(a-b)^{2}}{2} \epsilon_{i j k} \frac{n_{j}}{r^{3}} T_{k}+O\left(\frac{1}{r^{5}}\right)
$$

with $g=\exp (i \pi \hat{n} \cdot T)$, and so the leading term is of pure gauge form, i.e. it is flat. Therefore, we have that

$$
F_{i j} \sim O\left(\frac{1}{r^{4}}\right) \quad F_{0 i} \sim O\left(\frac{1}{r^{5}}\right)
$$

Consequently, the integrand in (4.1.4), namely $\left(\alpha F_{i j}+\beta \widetilde{F}_{i j}\right) \frac{\partial x^{i}}{\partial \sigma} \frac{\partial x^{j}}{\partial \tau}$, behaves as $O\left(\frac{1}{r^{2}}\right)$ in the limit $r \rightarrow \infty$. Therefore, the charges associated to (4.1.4) vanish, i.e. $Q_{S}=1$.

Note that in the limit $r \rightarrow \infty$ the spatial component of the connection (4.5.6) for the two-meron solution is twice that of the one-meron solution (4.5.1). Therefore, the evaluation 
of the charges associated to the operator (4.3.18) is very similar to that leading to (4.5.5) and gives

$$
V_{x_{\mathrm{R}}^{(t)}}^{\prime}\left(D_{\infty}^{(t)}\right)=P_{1} e^{-i e \oint_{S_{\infty}}(t) d \sigma A_{i} \frac{d x^{i}}{d \sigma}}=e^{-i 4 \pi T_{3}}=\mathbb{1}
$$

where the last equality follows from the fact that the leading term of $A_{i}$ is flat. The interpretation for such conserved charges, given below (4.3.18), holds true, i.e. they correspond to the magnetic flux through the surface which border is $S_{\infty}^{1,(t)}$, and such fluxes are also quantized.

The meron-antimeron solution in the Coulomb gauge, corresponding to a meron and an anti-meron located at $x^{\mu}=-a^{\mu}$ and $x^{\mu}=a^{\mu}$ respectively, with $a^{\mu}=(0,0,0, a)$, is given by (41, 44)

$$
A_{i}=-\frac{1}{e} \epsilon_{i j k} \frac{n_{j}}{r}\left(1-\frac{x^{2}-a^{2}}{\sqrt{(x+a)^{2}(x-a)^{2}}}\right) T_{k} \quad A_{4}=0
$$

Again expanding in powers of $\frac{1}{r}$ one gets

$$
A_{i}=-\frac{2 a^{2}}{e} \epsilon_{i j k} \frac{n_{j}}{r^{3}} T_{k}+O\left(\frac{1}{r^{5}}\right)
$$

Then, similarly to the two-meron case one has that $F_{i j} \sim O\left(\frac{1}{r^{4}}\right)$ and $F_{0 i} \sim O\left(\frac{1}{r^{5}}\right)$, and so the charges coming from (4.1.4) are trivial, i.e. $Q_{S}=1$. In addition, since the connection falls faster than $\frac{1}{r}$ the integrand in the operator (4.3.18) vanishes, i.e. $P_{1} e^{-i e \oint_{S_{\infty}^{1,(t)}} d \sigma A_{i} \frac{d x^{i}}{d \sigma}}=1$. The corresponding charges are also trivial in this case. 


\section{CHAPTER 5}

\section{Quasi-integrable deformation of the non-linear Schrödinger equation}

In this chapter we analyse the phenomenon of quasi-integrability through some deformations of the non-linear Schrödinger equation. In section [5.1 the models we study are described in details, the anomalous zero-curvature is constructed, the quasi-conserved charges are calculated and we establish the conditions that have to be satisfied by the solutions for the integrated anomalies to vanish. We also give an argument, valid in a space-time of any dimension, for a field theory to possess charges satisfying symmetries of the type given in (1.0.10). In section 5.2 we discuss how the dynamics of the model favours solutions satisfying (1.0.9). We also discuss further the relation between the dynamics and parity for the case when the potential is a deformation of the NLS potential. In section 5.3 we discuss the parity properties of the one and two-soliton solutions of the NLS theory and show how to select those that satisfy (1.0.9). We then present, in section 5.4, the results of our numerical simulations which support the analytical results discussed in the previous sections. The numerical simulations were a major contribution made by Wojciech Zakrzewski from the Durham University.In the appendix B we present the details of the calculation used in section 5.1, and in appendix C we use the Hirota method to construct one and two-bright-soliton solutions of the NLS theory.

\subsection{Definition of the model}

We consider a non-relativistic complex scalar field in $(1+1)$ dimensions whose dynamics is governed by the Lagrangian

$$
\mathcal{L}=\frac{i}{2}\left(\bar{\psi} \partial_{t} \psi-\psi \partial_{t} \bar{\psi}\right)-\partial_{x} \bar{\psi} \partial_{x} \psi-V\left(|\psi|^{2}\right)
$$

where $\bar{\psi}$ is the complex conjugate of $\psi$. The equations of motion are

$$
i \partial_{t} \psi=-\partial_{x}^{2} \psi+\frac{\partial V}{\partial|\psi|^{2}} \psi
$$


together with its complex conjugate. The corresponding "Hamiltonian" reads

$$
\mathcal{H}=\left|\partial_{x} \psi\right|^{2}+V\left(|\psi|^{2}\right)
$$

We shall consider solutions of (5.1.2) satisfying the following boundary conditions

$$
|\psi|_{x=-\infty}=|\psi|_{x=\infty} ; \quad \partial_{x} \psi \rightarrow 0 \quad \text { for } \quad x \rightarrow \pm \infty
$$

It is easy to check that the energy $E$, momentum $P$ and normalisation $N$ of the solutions of the equations of motion(5.1.2) satisfying (5.1.4), as defined below, are conserved in time:

$$
\begin{aligned}
E & =\int_{-\infty}^{\infty} d x\left(\left|\partial_{x} \psi\right|^{2}+V\right) \\
P & =i \int_{-\infty}^{\infty} d x\left(\bar{\psi} \partial_{x} \psi-\psi \partial_{x} \bar{\psi}\right) \\
N & =\int_{-\infty}^{\infty} d x|\psi|^{2} .
\end{aligned}
$$

In fact, these conserved quantities correspond to the Noether charges of the model. The energy $E$ is connected with the invariance of (5.1.1) under time translations, the momentum $P$ under the space translations, and $N$ is related to the $U(1)$ symmetry of the Lagrangian (5.1.1)

$$
\psi \rightarrow e^{i \alpha} \psi \quad \alpha \equiv \text { const. }
$$

The integrable Non-Linear Schrödinger theory (NLS) corresponds to the potential

$$
V_{\mathrm{NLS}}=\eta\left|\psi_{0}\right|^{4}
$$

which leads to the NLS equation

$$
i \partial_{t} \psi_{0}=-\partial_{x}^{2} \psi_{0}+2 \eta\left|\psi_{0}\right|^{2} \psi_{0}
$$

The sign of the parameter $\eta$ plays an important role in the physical properties of the solutions, and we refer to $(31-34)$ for a more detailed discussion of this point. Indeed, for $\eta<0$ we have the so-called bright soliton solutions given by

$$
\psi_{0}=\frac{|\rho|}{\sqrt{|\eta|}} \frac{e^{i\left[\left(\rho^{2}-\frac{v^{2}}{4}\right) t+\frac{v}{2} x\right]}}{\cosh \left[\rho\left(x-v t-x_{0}\right)\right]}
$$

with $\rho, v$ and $x_{0}$ being real parameters of the solution. For $\eta>0$ we have the dark soliton 
solution given by

$$
\psi_{0}=\frac{|\rho|}{\sqrt{\eta}} \tanh \left[\rho\left(x-v t-x_{0}\right)\right] e^{i\left[\frac{v}{2} x-\left(2 \rho^{2}+\frac{v^{2}}{4}\right) t\right]} .
$$

Note, that the solutions are defined up to an overall constant phase due to the symmetry (5.1.8).

The equation (5.1.2) admits an anomalous zero curvature representation (Lax-ZakharovShabat equation) with the connection given by*

$$
\begin{aligned}
& A_{x}=-i T_{3}^{1}+\bar{\gamma} \bar{\psi} T_{+}^{0}+\gamma \psi T_{-}^{0} \\
& A_{t}=i T_{3}^{2}+i \frac{\delta V}{\delta|\psi|^{2}} T_{3}^{0}-\left(\bar{\gamma} \bar{\psi} T_{+}^{1}+\gamma \psi T_{-}^{1}\right)-i\left(\bar{\gamma} \partial_{x} \bar{\psi} T_{+}^{0}-\gamma \partial_{x} \psi T_{-}^{0}\right)
\end{aligned}
$$

where the generators $T_{i}^{n}, i=3,+,-$, and $n$ integer, satisfy the so-called $S L(2)$ loop algebra commutation relations

$$
\left[T_{3}^{m}, T_{ \pm}^{n}\right]= \pm T_{ \pm}^{m+n} ; \quad\left[T_{+}^{m}, T_{-}^{n}\right]=2 T_{3}^{m+n}
$$

which can be realized in terms of the finite $S L(2)$ algebra generators as $T_{i}^{n} \equiv \lambda^{n} T_{i}$, with $\lambda$ an arbitrary complex parameter. The curvature of the connection (5.1.13) is given by

$$
\begin{aligned}
\partial_{t} A_{x}-\partial_{x} A_{t}+\left[A_{x}, A_{t}\right] & =X T_{3}^{0}+i \bar{\gamma}\left[-i \partial_{t} \bar{\psi}+\partial_{x}^{2} \bar{\psi}-\bar{\psi} \frac{\delta V}{\delta|\psi|^{2}}\right] T_{+}^{0} \\
& -i \gamma\left[i \partial_{t} \psi+\partial_{x}^{2} \psi-\psi \frac{\delta V}{\delta|\psi|^{2}}\right] T_{-}^{0}
\end{aligned}
$$

with

$$
X \equiv-i \partial_{x}\left(\frac{\delta V}{\delta|\psi|^{2}}-2 \gamma \bar{\gamma}|\psi|^{2}\right)
$$

In consequence, when the equations of motion (5.1.2) are imposed, the terms on the r.h.s. of (5.1.15), proportional to $T_{+}^{0}$ and $T_{-}^{0}$, vanish. Note also that by taking

$$
\eta \equiv \gamma \bar{\gamma}
$$

the anomaly $X$, given in (5.1.16), vanishes for the NLS potential (5.1.9), and so the curvature (5.1.15) vanishes, which makes the NLS equation integrable.

In this thesis we discuss a generalisation of this theory (i.e., deformations of the NLS potential) which makes the resultant theory non-integrable (the anomaly (5.1.16) does not vanish), but, as we will show, it exhibit properties very similar to the integrable theory, like

*As it will become clear later, $\bar{\gamma}$ is not necessarily the complex conjugate of $\gamma$. 
the solitons preserving their shapes after the scattering etc. In addition, we will show, using the algebraic techniques borrowed from integrable field theories, that the anomalous LaxZakharov-Shabat equation (5.1.15) leads to an infinite number of quasi-conservation laws and we will find that, under some special circumstances, the corresponding charges are conserved asymptotically in the scattering of soliton type solutions of the deformed theory.

In order, to employ the algebraic techniques mentioned above it is more convenient to work with a new basis of the $S L(2)$ loop algebra and a new parametrisation of the fields. We shall use the modulus $R$ of $\psi^{2}$ and its phase $\varphi$, defined by

$$
\psi=\sqrt{R} e^{i \frac{\varphi}{2}} .
$$

In addition, the complex parameters $\gamma$ and $\bar{\gamma}$, appearing in the connection (5.1.13), are written as

$$
\gamma=i \sqrt{|\eta|} e^{i \phi}, \quad \bar{\gamma}=-i \sigma \sqrt{|\eta|} e^{-i \phi}, \quad \gamma \bar{\gamma}=\eta, \quad \sigma=\operatorname{sign} \eta
$$

The new basis of the $S L(2)$ loop algebraic is then defined as

$$
b_{n}=T_{3}^{n}, \quad F_{1}^{n}=\frac{1}{2}\left(\sigma T_{+}^{n}-T_{-}^{n}\right), \quad F_{2}^{n}=\frac{1}{2}\left(\sigma T_{+}^{n}+T_{-}^{n}\right),
$$

which satisfy

$$
\left[b_{m}, b_{n}\right]=0 ; \quad\left[b_{n}, F_{1}^{m}\right]=F_{2}^{n+m} ; \quad\left[b_{n}, F_{2}^{m}\right]=F_{1}^{n+m} ; \quad\left[F_{1}^{n}, F_{2}^{m}\right]=\sigma b_{n+m}
$$

As usual we perform the gauge transformation

$$
A_{\mu} \rightarrow \tilde{A}_{\mu} \equiv \tilde{g} A_{\mu} \tilde{g}^{-1}+\partial_{\mu} \tilde{g} \tilde{g}^{-1} ; \quad \text { with } \quad \tilde{g}=e^{i\left(\frac{\varphi}{2}+\phi\right) b_{0}}
$$

and find that the connection (5.1.13) has now become

$$
\begin{aligned}
\tilde{A}_{x} & =-i b_{1}+\frac{i}{2} \partial_{x} \varphi b_{0}-2 i \sqrt{|\eta|} \sqrt{R} F_{1}^{0} \\
\tilde{A}_{t} & =i b_{2}+\frac{i}{2} \partial_{t} \varphi b_{0}+i \frac{\delta V}{\delta R} b_{0}+2 i \sqrt{|\eta|} \sqrt{R} F_{1}^{1} \\
& +\sqrt{|\eta|} \sqrt{R}\left[-\frac{\partial_{x} R}{R} F_{2}^{0}+i \partial_{x} \varphi F_{1}^{0}\right]
\end{aligned}
$$

For the fields which satisfy the equations of motion (5.1.2) the curvature becomes

$$
\tilde{F}_{t x}=\partial_{t} \tilde{A}_{x}-\partial_{x} \tilde{A}_{t}+\left[\tilde{A}_{x}, \tilde{A}_{t}\right]=X b_{0} ; \quad \text { with } \quad X \equiv-i \partial_{x}\left(\frac{\delta V}{\delta R}-2 \eta R\right)
$$


To go further we carry out the usual abelianisation technique of the integrable field theories (2, 28-30); i.e., we perform a further gauge transformation

$$
\tilde{A}_{\mu} \rightarrow a_{\mu}=g \tilde{A}_{\mu} g^{-1}+\partial_{\mu} g g^{-1}
$$

with

$$
g=e^{\sum_{n=1}^{\infty} \mathcal{F}^{(-n)}} ; \quad \text { where } \quad \mathcal{F}^{(-n)} \equiv \zeta_{1}^{(-n)} F_{1}^{-n}+\zeta_{2}^{(-n)} F_{2}^{-n}
$$

The parameters $\zeta_{i}^{(-n)}$ are chosen, as we will explain below, so that the $a_{x}$ component of the transformed connection lies in the infinite abelian sub-algebra spanned by the generators $b_{n}$.

An important role in our construction is played by the grading operator $d$ defined as

$$
d \equiv \lambda \frac{d}{d \lambda}, \quad\left[d, b_{n}\right]=n b_{n}, \quad\left[d, F_{i}^{n}\right]=n F_{i}^{n} .
$$

The $\tilde{A}_{x}$ component of the connection (5.1.23) has generators of grade 0 and 1 . Since the group element (5.1.26) is an exponentiation of negative grade generators, the $a_{x}$ component of the transformed connection has generators of grades ranging from 1 to $-\infty$. Splitting the transformed potential (5.1.25) into its eigen-sub-spaces under the grading operator (5.1.27), i.e., $a_{x}=\sum_{n=1}^{\infty} a_{x}^{(n)}$, we find that

$$
\begin{aligned}
a_{x}^{(1)} & =-i b_{1} \\
a_{x}^{(0)} & =i\left[b_{1}, \mathcal{F}^{(-1)}\right]+\tilde{A}_{x}^{(0)} \\
a_{x}^{(-1)} & =i\left[b_{1}, \mathcal{F}^{(-2)}\right]+\left[\mathcal{F}^{(-1)}, \tilde{A}_{x}^{(0)}\right]-\frac{i}{2 !}\left[\mathcal{F}^{(-1)},\left[\mathcal{F}^{(-1)}, b_{1}\right]\right] \\
& +\partial_{x} \mathcal{F}^{(-1)}, \\
a_{x}^{(-2)} & =i\left[b_{1}, \mathcal{F}^{(-3)}\right]+\left[\mathcal{F}^{(-2)}, \tilde{A}_{x}^{(0)}\right]-\frac{i}{2 !}\left[\mathcal{F}^{(-2)},\left[\mathcal{F}^{(-1)}, b_{1}\right]\right] \\
& -\frac{i}{2 !}\left[\mathcal{F}^{(-1)},\left[\mathcal{F}^{(-2)}, b_{1}\right]\right]+\frac{1}{2 !}\left[\mathcal{F}^{(-1)},\left[\mathcal{F}^{(-1)}, \tilde{A}_{x}^{(0)}\right]\right] \\
& -\frac{i}{3 !}\left[\mathcal{F}^{(-1)},\left[\mathcal{F}^{(-1)},\left[\mathcal{F}^{(-1)}, b_{1}\right]\right]\right] \\
& +\partial_{x} \mathcal{F}^{(-2)}+\frac{1}{2 !}\left[\mathcal{F}^{(-1)}, \partial_{x} \mathcal{F}^{(-1)}\right]
\end{aligned}
$$

where we have denoted $\tilde{A}_{x}^{(0)}=\frac{i}{2} \partial_{x} \varphi b_{0}-2 i \sqrt{|\eta|} \sqrt{R} F_{1}^{0}$ (see (15.1.25) $)$.

An important ingredient of this construction is the observation that the generator $b_{1}$ is a semi-simple element (in fact any $b_{n}$ is) in the sense that it splits the $S L(2)$ loop algebra $\mathcal{G}$ 
into the kernel (Ker) and image $(\mathrm{Im})$ of its adjoint action, i.e.

$$
\mathcal{G}=\text { Ker }+\operatorname{Im} ; \quad \text { with } \quad\left[b_{1}, \text { Ker }\right]=0 ; \quad \operatorname{Im}=\left[b_{1}, \mathcal{G}\right] .
$$

The Ker and Im sub-spaces do not have common elements, i.e. any element of $\mathcal{G}$ commuting with $b_{1}$ cannot be written as a commutator of $b_{1}$ with some other element of $\mathcal{G}$. One notes from (5.1.21) that $b_{n}$ constitute a basis of Ker, and $F_{i}^{n}, i=1,2$, a basis of Im. In addition, one notes from (15.1.28) that the first time that $\mathcal{F}^{(-n)}$ appears in the expansion of $a_{x}$, is in the component $a_{x}^{-n+1}$ of grade $-n+1$, and it appears in the form $\left[b_{1}, \mathcal{F}^{(-n)}\right]$. Therefore, one can choose the parameters in $\mathcal{F}^{(-n)}$ so that they cancel the image component of $a_{x}^{-n+1}$. This can be done recursively starting at the component of grade 0 and working downwards. It is then clear that the gauge transformation (5.1.26) can rotate the $a_{x}$ component of the connection into the Abelian sub-algebra generated by the $b_{n}$ 's, i.e.,

$$
a_{x}=-i b_{1}+\sum_{n=0}^{\infty} a_{x}^{(3,-n)} b_{-n} .
$$

Note from (5.1.23) that $\tilde{A}_{x}$ depends on the real fields $R$ and $\partial_{x} \varphi$. Thus, the components $a_{x}^{(3, n)}$ are polynomials in these fields and their $x$-derivatives, and they do not depend on the potential $V$. In consequence, the $a_{x}$ component of the connection is the same for any choice of the potential. In the appendix $(\mathbb{B})$ we give explicit expressions for the first few components of $a_{x}$.

On the other hand the $\tilde{A}_{t}$ component of the connection (5.1.23) depends on the choice of the potential $V$. In fact, for the case of the NLS potential (5.1.9) we note that the gauge transformation (5.1.25), with the group element (5.1.26) fixed as above, does rotate $a_{t}$ into an Abelian sub-algebra generated by the $b_{n}$ 's, when the equations of motion (5.1.10) are satisfied. For other choices of $V$ this does not take place even when the equations of motion (5.1.2) are imposed. Thus, we find that

$$
a_{t}=i b_{2}+\sum_{n=0}^{\infty}\left[a_{t}^{(3,-n)} b_{-n}+a_{t}^{(1,-n)} F_{1}^{-n}+a_{t}^{(2,-n)} F_{2}^{-n}\right] .
$$

Next we note that $a_{t}$ does not have the grade 1 component due to the fact that the coefficient of $F_{1}^{0}$ in $\tilde{A}_{x}$, and the coefficient of $F_{1}^{1}$ in $\tilde{A}_{t}$, are the same up to a sign (see ([5.1.23) $)$. Under the gauge transformation (5.1.25) the curvature $F_{t x}$ transforms to $F_{t x} \rightarrow g F_{t x} g^{-1}$, and so from (5.1.24) we see that

$$
\partial_{t} a_{x}-\partial_{x} a_{t}+\left[a_{t}, a_{x}\right]=X g b_{0} g^{-1}
$$


Since $a_{x}$ lies in the kernel of $b_{1}$ it follows that $\left[a_{t}, a_{x}\right]$ has components only in the image of $b_{1}$. Thus, denoting

$$
g b_{0} g^{-1}=\sum_{n=0}^{\infty}\left[\alpha^{(3,-n)} b_{-n}+\alpha^{(1,-n)} F_{1}^{-n}+\alpha^{(2,-n)} F_{2}^{-n}\right]
$$

we find that

$$
\partial_{t} a_{x}^{(3,-n)}-\partial_{x} a_{t}^{(3,-n)}=X \alpha^{(3,-n)} ; \quad n=0,1,2, \ldots
$$

The explicit expressions for the first few $\alpha^{(i,-n)}, i=1,2,3$, are given in appendix B. Note that if the time component of the connection satisfies the boundary condition $a_{t}^{(3,-n)}(x=\infty)=a_{t}^{(3,-n)}(x=-\infty)$, which is the case in the example we consider, then we have anomalous conservation laws

$\frac{d Q^{(n)}}{d t}=\beta_{n} ; \quad$ with $\quad Q^{(n)}=\int_{-\infty}^{\infty} d x a_{x}^{(3,-n)} ; \quad$ where $\quad \beta_{n}=\int_{-\infty}^{\infty} d x X \alpha^{(3,-n)}$

Of course, in the case of the NLS theory we get an infinite number of conserved quantities since the anomaly $X$, given in (5.1.16) or (5.1.24), vanishes for the NLS potential (5.1.9).

We now use a more refined algebraic technique to explore the structure of the anomalies $\beta_{n}$. The key ingredients are the two $\mathbb{Z}_{2}$ transformations, one in the internal space of the loop algebra and the other in space-time. The first $\mathbb{Z}_{2}$ transformation is an order 2 automorphism of the $S L(2)$ loop algebra (5.1.21) given by

$$
\Sigma\left(b_{n}\right)=-b_{n}, \quad \Sigma\left(F_{1}^{n}\right)=-F_{1}^{n}, \quad \Sigma\left(F_{2}^{n}\right)=F_{2}^{n} .
$$

The second $\mathbb{Z}_{2}$ transformation is a space-time reflection around a given point $\left(x_{\Delta}, t_{\Delta}\right)$, i.e.,

$$
P: \quad(\tilde{x}, \tilde{t}) \rightarrow(-\tilde{x},-\tilde{t}) \quad \text { with } \quad \tilde{x}=x-x_{\Delta} \quad \tilde{t}=t-t_{\Delta}
$$

Consider now solutions of the equations of motion (5.1.2) of the theory (5.1.1) such that, in addition, they satisfy the following property under the parity (5.1.37) (see (5.1.18))

$$
P: \quad R \rightarrow R ; \quad \varphi \rightarrow-\varphi+\text { constant. }
$$

Then, the $x$-component of the connection (5.1.23) transforms as

$$
\Sigma\left(\tilde{A}_{x}\right)=-\tilde{A}_{x} \quad P\left(\tilde{A}_{x}\right)=\tilde{A}_{x}
$$


and so it is odd under the joint action of the two $\mathbb{Z}_{2}$ transformations:

$$
\Omega\left(\tilde{A}_{x}\right)=-\tilde{A}_{x}, \quad \Omega \equiv \Sigma P .
$$

In fact, this property is valid for every individual component of $\tilde{A}_{x}$. Thus we see that we have $\Omega\left(\left[b_{1}, \mathcal{F}^{(-n)}\right]\right)=-\left[b_{1}, \Omega\left(\mathcal{F}^{(-n)}\right)\right]$, and so

$$
(1+\Omega)\left(\left[b_{1}, \mathcal{F}^{(-n)}\right]\right)=\left[b_{1},(1-\Omega) \mathcal{F}^{(-n)}\right]
$$

Since $\tilde{A}_{x}^{(0)}$ is odd under $\Omega$, it follows from the second equation of $(\underline{5.1 .28})$ that

$$
(1+\Omega) a_{x}^{(0)}=i\left[b_{1},(1-\Omega) \mathcal{F}^{(-1)}\right] .
$$

The r.h.s. of (5.1.42) is clearly in the image of the adjoint action, and we have chosen the $\mathcal{F}^{(-n)}$ to rotate $a_{x}$ into the kernel of that same adjoint action. Therefore, the only possibility for (5.1.42) to hold is that both sides vanish, i.e., that

$$
(1+\Omega) a_{x}^{(0)}=0, \quad(1-\Omega) \mathcal{F}^{(-1)}=0
$$

and so that $\mathcal{F}^{(-1)}$ is even under $\Omega$. Using this fact we see from the third equation in (5.1.28) that

$$
(1+\Omega) a_{x}^{(-1)}=i\left[b_{1},(1-\Omega) \mathcal{F}^{(-2)}\right]
$$

Furthermore, using same arguments we conclude also that

$$
(1+\Omega) a_{x}^{(-1)}=0, \quad(1-\Omega) \mathcal{F}^{(-2)}=0
$$

and so that $\mathcal{F}^{(-2)}$ is even under $\Omega$ as well. Again, from the fourth equation in (5.1.28) we see that $(1+\Omega) a_{x}^{(-2)}=i\left[b_{1},(1-\Omega) \mathcal{F}^{(-3)}\right]$, and so by the same arguments as before we conclude that

$$
(1+\Omega) a_{x}^{(-2)}=0, \quad(1-\Omega) \mathcal{F}^{(-3)}=0
$$

Repeating this reasoning we reach the conclusion that all $\mathcal{F}^{(-n)}$ are even under $\Omega$. So, the group element $g$, given in (5.1.26), is even under $\Omega$

$$
\Omega(g)=g
$$

To go further we note that since $\tilde{A}_{x}$ and $\partial_{x}$ are odd under $\Omega$, and since $g$ is even (5.1.25) demonstrates that $a_{x}$ has to be odd under $\Omega$. One can verify all these claims by inspecting the explicit expressions for the parameters $\zeta_{i}^{(-n)}$ given in appendix $\mathbb{B}$. Since the $\mathcal{F}^{(-n)}$ are even 
under $\Omega$, and since the generators satisfy (5.1.36), it follows from (5.1.26) that $P\left(\zeta_{1}^{(-n)}\right)=$ $-\zeta_{1}^{(-n)}$ and $P\left(\zeta_{2}^{(-n)}\right)=\zeta_{2}^{(-n)}$.

Next we use the Killing form of the $S L(2)$ loop algebra given by

$$
\operatorname{Tr}\left(b_{n} b_{m}\right)=\frac{1}{2} \delta_{n+m, 0} ; \quad \operatorname{Tr}\left(b_{n} F_{i}^{m}\right)=0 ; \quad i=1,2,
$$

which can be realized by $\operatorname{Tr}(\star) \equiv \frac{1}{2 \pi i} \oint \frac{d \lambda}{\lambda} \operatorname{tr}(\star)$, with tr being the ordinary finite matrix trace, and $T_{i}^{n}=\lambda T_{i}, i=3, \pm$. In this case we see from (5.1.33) that

$$
\alpha^{(3,-n)}=2 \operatorname{Tr}\left(g b_{0} g^{-1} b_{n}\right)=2 \operatorname{Tr}\left(\Sigma(g) b_{0} \Sigma\left(g^{-1}\right) b_{n}\right)
$$

where in the last equality we have used the fact that the Killing form is invariant under $\Sigma$, and that all the $b_{n}$ 's are odd under it. Thus, using (5.1.47) we have that

$$
P\left(\alpha^{(3,-n)}\right)=2 \operatorname{Tr}\left(\Omega(g) b_{0} \Omega\left(g^{-1}\right) b_{n}\right)=2 \operatorname{Tr}\left(g b_{0} g^{-1} b_{n}\right)=\alpha^{(3, n)}
$$

and so we see that all the $\alpha^{(3,-n)}$ 's are even under $P$. Note that $X$, given in (5.1.24), is an $x$-derivative of a functional of $R$. Since we have assumed that $R$ is even under $P$, we see from (5.1.38) that $X$ is odd, i.e., that $P(X)=-X$ and so that

$$
\int_{-\tilde{t}_{0}}^{\tilde{t}_{0}} d t \int_{-\tilde{x}_{0}}^{\tilde{x}_{0}} d x X \alpha^{(3,-n)}=0,
$$

where $\tilde{t}_{0}$ and $\tilde{x}_{0}$ are given fixed values of the shifted time $\tilde{t}$ and space coordinate $\tilde{x}$ respectively, introduced in (5.1.37). Therefore, by taking $\tilde{x}_{0} \rightarrow \infty$, we conclude that the non-conserved charges (5.1.35) satisfy the following mirror time-symmetry around the point: $t_{\Delta}$.

$$
Q^{(n)}\left(t=\tilde{t}_{0}+t_{\Delta}\right)=Q^{(n)}\left(t=-\tilde{t}_{0}+t_{\Delta}\right)
$$

In consequence, even though the charges $Q^{(n)}$ vary in time, they are symmetric with respect to $t=t_{\Delta}$. Note that we have derived this property for any potential $V$ which depends only on the modulus of $\psi$. The only assumption we have made is that we are considering fields $\psi$ which satisfy (5.1.38).

In the next sections we will show that such solutions are very plausible and that, in fact, the one and two-soliton solutions of the theories (5.1.1) can always be chosen to satisfy (5.1.38). This fact has far reaching consequences for the properties of the theories (5.1.1). For instance, by taking $\tilde{t}_{0} \rightarrow \infty$ one concludes that the scattering of two-soliton solutions presents an infinite number of charges which are asymptotically conserved. Since the $S$-matrix relies only on asymptotic states, it is quite plausible that the theories (5.1.1) share a lot of 
interesting properties with integrable theories (but which have been believed to be only true for integrable field theories).

\subsubsection{On the parity symmetry}

The properties leading to charges satisfying (5.1.52) can be realised in a much wider context. Indeed, consider a field theory in a space-time of $(d+1)$ dimensions with fields labelled by $\varphi_{a}, a=1,2, \ldots n$. These fields can be scalars, vectors, spinors, etc., and the indices $a$ just label their components. Consider a fixed point $x_{\Delta}^{\mu}$ in space-time, and a reflection $P$ around it, i.e.,

$$
P: \quad \tilde{x}^{\mu} \rightarrow-\tilde{x}^{\mu} \quad \text { with } \quad \tilde{x}^{\mu}=x^{\mu}-x_{\Delta}^{\mu} \quad \mu=0,1,2 \ldots d .
$$

Suppose that a such field theory possesses a classical solution $\varphi_{a}^{s}$ such that the fields evaluated on it are eigenvectors of $P$ up to constants, i.e. that

$$
P\left(\varphi_{a}^{s}\right)=\varepsilon_{a} \varphi_{a}^{s}+c_{a}, \quad \varepsilon_{a}= \pm 1 ; \quad c_{a}=\text { const. }
$$

Consider now a functional of the fields and of their derivatives $F=F\left(\varphi_{a}, \partial_{\mu} \varphi_{a}, \partial_{\mu} \partial_{\nu} \varphi_{a}, \ldots\right)$, that is even under $P$ when evaluated on a particular solution, i.e.

$$
P\left[F\left(\varphi_{a}^{s}, \partial_{\mu} \varphi_{a}^{s}, \partial_{\mu} \partial_{\nu} \varphi_{a}^{s}, \ldots\right)\right]=F\left(\varphi_{a}^{s}, \partial_{\mu} \varphi_{a}^{s}, \partial_{\mu} \partial_{\nu} \varphi_{a}^{s}, \ldots\right)
$$

Next, look at a rectangular spatial volume $\mathcal{V}$ bounded by hyperplanes crossing the axes of the space coordinates at the points $\pm \tilde{x}_{0}^{i}, i=1,2, \ldots d$, corresponding to fixed values of the shifted space coordinates $\tilde{x}^{i}$ introduced in (5.1.53), i.e. such that the point $x_{\Delta}^{i}$ lies in the very centre of $\mathcal{V}$. The integral of this functional over $\mathcal{V}$

$$
Q=\int_{\mathcal{V}} d^{d} x F
$$

satisfies

$$
\frac{d Q}{d x^{0}}=\int_{\mathcal{V}} d^{d} x \frac{d F}{d x^{0}}=\int_{\mathcal{V}} d^{d} x\left[\frac{\delta F}{\delta \varphi_{a}} \partial_{0} \varphi_{a}+\frac{\delta F}{\delta \partial_{\mu} \varphi_{a}} \partial_{0} \partial_{\mu} \varphi_{a}+\frac{\delta F}{\delta \partial_{\mu} \partial_{\nu} \varphi_{a}} \partial_{0} \partial_{\mu} \partial_{\nu} \varphi_{a}+\ldots\right] .
$$

When evaluated on the solution $\varphi_{a}^{s}$ each term in the integrand in (5.1.57) is odd under $P$. The reasons for this are simple: any derivative of the form $\partial_{0} \partial_{\mu_{1}} \ldots \partial_{\mu_{m}} \varphi_{a}$, when evaluated on $\varphi_{a}^{s}$, has an eigenvalue of $P$ equal to $\varepsilon_{a}(-1)^{m+1}$. Since $F$ evaluated on $\varphi_{a}^{s}$ is even under $P$, it follows that any derivative of the form $\frac{\delta F}{\delta \partial_{\mu_{1} \ldots \partial_{m} \varphi_{a}}}$ has an eigenvalue of $P$ equal to $\varepsilon_{a}(-1)^{m}$, when evaluated on $\varphi_{a}^{s}$. Therefore, when evaluated on $\varphi_{a}^{s}$ each term of the integrand on the 
r.h.s. of (5.1.57) is odd under $P$. Consequently, one finds that

$$
\begin{aligned}
Q^{s}\left(\tilde{x}^{0}\right) & -Q^{s}\left(-\tilde{x}^{0}\right)=\int_{-\tilde{x}^{0}}^{\tilde{x}^{0}} d x^{0} \frac{d Q^{s}}{d x^{0}} \\
& =\int_{-\tilde{x}^{0}}^{\tilde{x}^{0}} d x^{0} \int_{\mathcal{V}} d^{d} x\left[\frac{\delta F^{s}}{\delta \varphi_{a}^{s}} \partial_{0} \varphi_{a}^{s}+\frac{\delta F^{s}}{\delta \partial_{\mu} \varphi_{a}^{s}} \partial_{0} \partial_{\mu} \varphi_{a}^{s}+\frac{\delta F^{s}}{\delta \partial_{\mu} \partial_{\nu} \varphi_{a}^{s}} \partial_{0} \partial_{\mu} \partial_{\nu} \varphi_{a}^{s}+\ldots\right]=0,
\end{aligned}
$$

where the superscript $s$ denotes that $Q$ is evaluated on the solution $\varphi_{a}^{s}$, and $\tilde{x}^{0}$ is a given fixed value of the shifted time introduced in (5.1.53).

Summarizing: if one has a solution of the theory such that all the fields evaluated on this solution are eigenstates of $P$, i.e. they satisfy (5.1.54), then any even functional of these fields and their derivatives leads to charges that satisfy a mirror time-symmetry like (5.1.58).

In the case studied in this thesis we have shown that the $x$-component of the connection, $a_{x}$, is odd under the transformation $\Omega=\Sigma P$, i.e. $(1+\Omega) a_{x}=0$. Since $a_{x}$ lies in the abelian subalgebra generated by the $b_{n}$ 's (see (5.1.30) ), which are odd under $\Sigma($ see (5.1.36) $)$, it follows that the charge densities $a_{x}^{(3,-n)}$ are even under $P$. Therefore, the charges $Q^{(n)}$ introduced in (5.1.35) are in the class of charges (5.1.56) discussed in this subsection. So, the assumption of the existence of a solution satisfying (5.1.38) has much deeper consequences. It implies not only that the charges (5.1.35) satisfy the mirror time-symmetry (5.1.52), but also that any charge built out of a density that is even under $P$ when evaluated on this solution, also satisfies (5.1.52). The fact that a solution satisfies (5.1.38) implies that its past and future w.r.t. to the point in time $t_{\Delta}$, are strongly linked and, in consequence, so are many of its properties. Indeed, the mirror time-symmetry (5.1.52) is a direct consequence of such a link between the past and the future. The non-linear phenomena behind the quasi-integrability properties we are discussing are certainly driven by the parity property (5.1.38). However, we still have to understand the basic physical processes guarantee that a given solution satisfies (5.1.38). This is one of the great challenges for our techniques to understand. In the next section, we argue that for the theories (5.1.1) for which the potential $V$ is a deformation of the NLS potential (5.1.9), the solutions satisfying (5.1.38) are favoured by the dynamics if the corresponding undeformed solution of the integrable NLS theory also satisfies (5.1.38). 


\subsection{Dynamics versus parity}

In terms of fields $R$ and $\varphi$ introduced in (5.1.18), the equations of motion (5.1.2) become

$$
\begin{aligned}
\partial_{t} R & =-\partial_{x}\left(R \partial_{x} \varphi\right) \\
-R^{2} \partial_{t} \varphi & =-R \partial_{x}^{2} R+\frac{R^{2}}{2}\left(\partial_{x} \varphi\right)^{2}+\frac{1}{2}\left(\partial_{x} R\right)^{2}+2 R^{2} \frac{\partial V}{\partial R} .
\end{aligned}
$$

Let us analyze what type of solutions these equations admit if we assume that the fields of these solutions are eigenstates of the of parity transformation $P$ introduced in (5.1.37). We split the fields as

$$
R=R^{(+)}+R^{(-)} ; \quad \varphi=\varphi^{(+)}+\varphi^{(-)},
$$

where

$$
P\left(R^{( \pm)}\right)= \pm R^{( \pm)}, \quad P\left(\varphi^{( \pm)}\right)= \pm \varphi^{( \pm)}+\text {constant }
$$

Let us now assume that we have a solution for which $R^{(+)}=\varphi^{(-)}=0$. Then, the I.h.s. of the first equation in (5.2.1) is even under $P$, and its r.h.s. is odd. Thus, $\partial_{t} R^{(-)}=0$ and $\partial_{x}\left(R^{(-)} \partial_{x} \varphi^{(+)}\right)=0$. In addition, the second equation in (5.2.1) implies that $\partial_{t} \varphi^{(+)}=$ $-2\left[\frac{\partial V}{\partial R}\right]^{(-)}$.

Note also that if we have a solution for which $R^{(-)}=\varphi^{(-)}=0$ we get very similar results, namely that $\partial_{t} R^{(+)}=0, \partial_{x}\left(R^{(+)} \partial_{x} \varphi^{(+)}\right)=0$, and that $\partial_{t} \varphi^{(+)}=-2\left[\frac{\partial V}{\partial R}\right]^{(-)}$.

In a similar way, if we assume that our solution satisfies $R^{(+)}=\varphi^{(+)}=0$, then the second equation in (5.2.1) implies that $\left[\frac{\partial V}{\partial R}\right]^{(-)}=0$. This condition, however, excludes potentials that are even functions of $R$, like the integrable NLS potential (5.1.9). Thus, we would not expect interesting non-trivial solutions, like a two-soliton solution, with one of these three classes of cases in which the fields are eigenstates of $P$.

The only remaining case is the one we assumed in (5.1.38), namely, that $R^{(-)}=\varphi^{(+)}=0$. One can easily check that the equations (5.2.1) do not impose any restrictions on the solutions of this type. Indeed, $\frac{\partial V}{\partial R}$ is always even under $P$ for any $V$, if $R$ is even under $P$.

Consequently, we would expect most of the interesting non-trivial results for solutions of the theories (5.1.1), for which the fields evaluated on them are eigenstates of $P$, to fall into the class (5.1.38). Of course, there can also exist classes of non-trivial solutions for which the parity components are mixed and the above arguments do not apply. However, this does not mean that the results of these arguments are necessarily incorrect. Sometimes they may still hold even though one has to work harder to prove them. In the next section we present 
a detailed analysis of the case in which the potential $V$ is a deformation of the NLS potential (5.1.9). Our analysis shows that the mixed solutions can always be "gauged away", order by order, in the perturbation expansion around the NLS theory.

\subsubsection{Deformations of the NLS theory}

We now consider the theories (5.1.1) for which the potential $V$ is a deformation of the NLS potential (5.1.9). The deformation is introduced through a parameter $\varepsilon$ such that for $\varepsilon=0, V$ corresponds to (5.1.9). We will not consider here the deformations for which the potential depends upon the phase of $\psi$. Examples of such potentials are

$$
V^{(1)}=\eta R^{2+\varepsilon} ; \quad V^{(2)}=\eta R^{2}+\varepsilon R^{3} ; \quad V^{(3)}=\eta R^{2} e^{-\varepsilon R},
$$

where $R=|\psi|^{2}$ was introduced in (5.1.18).

We start our analysis by expanding the solutions of the corresponding equations of motion in powers of $\varepsilon$ around the solution of the integrable NLS theory as

$$
R=R_{0}+\varepsilon R_{1}+\varepsilon^{2} R_{2}+\ldots, \quad \varphi=\varphi_{0}+\varepsilon \varphi_{1}+\varepsilon^{2} \varphi_{2}+\ldots
$$

Of course, the deformed potential $V$ has the expansion

$$
V=\left.V\right|_{\varepsilon=0}+\varepsilon\left[\left.\frac{\partial V}{\partial \varepsilon}\right|_{\varepsilon=0}+\left.\frac{\partial V}{\partial R}\right|_{\varepsilon=0} R_{1}\right]+O\left(\varepsilon^{2}\right)
$$

and its gradient has the expansion

$$
\begin{aligned}
\frac{\partial V}{\partial R} & =\left.\frac{\partial V}{\partial R}\right|_{\varepsilon=0}+\varepsilon\left[\left.\frac{\partial^{2} V}{\partial \varepsilon \partial R}\right|_{\varepsilon=0}+\left.\frac{\partial^{2} V}{\partial R^{2}}\right|_{\varepsilon=0} R_{1}\right] \\
& +\frac{\varepsilon^{2}}{2}\left[\left.\frac{\partial^{3} V}{\partial \varepsilon^{2} \partial R}\right|_{\varepsilon=0}+\left.2 \frac{\partial^{3} V}{\partial \varepsilon \partial R^{2}}\right|_{\varepsilon=0} R_{1}+\left.\frac{\partial^{3} V}{\partial R^{3}}\right|_{\varepsilon=0} R_{1}^{2}+\left.2 \frac{\partial^{2} V}{\partial R^{2}}\right|_{\varepsilon=0} R_{2}\right]+\ldots
\end{aligned}
$$

We also expand the equations of motion (5.2.1) into powers of $\varepsilon$ and at the same time we split the equations, and so the fields, into their even and odd parts under a given space-time parity $P$ of the type (5.1.37). At this stage the value of the point $\left(x_{\Delta}, t_{\Delta}\right)$ around which we perform the reflection is not yet important. We just use the fact that the operation $P$ satisfies $P^{2}=1$, and so it has eigenvalues \pm 1 . Next we introduce the following notation for the eigen-components of the fields:

$$
\star^{( \pm)} \equiv \frac{1}{2}(1 \pm P) \star .
$$


Then the zero order part of the equations of motion (5.2.1) splits under $P$ as

$$
\begin{aligned}
& \partial_{t} R_{0}^{(-)}=-\partial_{x}\left(R_{0}^{(+)} \partial_{x} \varphi_{0}^{(+)}+R_{0}^{(-)} \partial_{x} \varphi_{0}^{(-)}\right), \\
& \partial_{t} R_{0}^{(+)}=-\partial_{x}\left(R_{0}^{(+)} \partial_{x} \varphi_{0}^{(-)}+R_{0}^{(-)} \partial_{x} \varphi_{0}^{(+)}\right)
\end{aligned}
$$

and

$$
\begin{aligned}
& -\left(\left(R_{0}^{(+)}\right)^{2}+\left(R_{0}^{(-)}\right)^{2}\right) \partial_{t} \varphi_{0}^{(-)}-2 R_{0}^{(+)} R_{0}^{(-)} \partial_{t} \varphi_{0}^{(+)}=-R_{0}^{(+)} \partial_{x}^{2} R_{0}^{(+)}-R_{0}^{(-)} \partial_{x}^{2} R_{0}^{(-)} \\
& +\frac{1}{2}\left(\left(R_{0}^{(+)}\right)^{2}+\left(R_{0}^{(-)}\right)^{2}\right)\left(\left(\partial_{x} \varphi_{0}^{(+)}\right)^{2}+\left(\partial_{x} \varphi_{0}^{(-)}\right)^{2}\right)+2 R_{0}^{(+)} R_{0}^{(-)} \partial_{x} \varphi_{0}^{(+)} \partial_{x} \varphi_{0}^{(-)} \\
& +\frac{1}{2}\left(\left(\partial_{x} R_{0}^{(+)}\right)^{2}+\left(\partial_{x} R_{0}^{(-)}\right)^{2}\right) \\
& +2\left(\left(R_{0}^{(+)}\right)^{2}+\left(R_{0}^{(-)}\right)^{2}\right)\left[\left.\frac{\partial V}{\partial R}\right|_{\varepsilon=0}\right]^{(+)}+4 R_{0}^{(+)} R_{0}^{(-)}\left[\left.\frac{\partial V}{\partial R}\right|_{\varepsilon=0}\right]^{(-)}
\end{aligned}
$$

and

$$
\begin{aligned}
& -\left(\left(R_{0}^{(+)}\right)^{2}+\left(R_{0}^{(-)}\right)^{2}\right) \partial_{t} \varphi_{0}^{(+)}-2 R_{0}^{(+)} R_{0}^{(-)} \partial_{t} \varphi_{0}^{(-)}=-R_{0}^{(+)} \partial_{x}^{2} R_{0}^{(-)}-R_{0}^{(-)} \partial_{x}^{2} R_{0}^{(+)} \\
& +\left(\left(R_{0}^{(+)}\right)^{2}+\left(R_{0}^{(-)}\right)^{2}\right) \partial_{x} \varphi_{0}^{(+)} \partial_{x} \varphi_{0}^{(-)}+R_{0}^{(+)} R_{0}^{(-)}\left(\left(\partial_{x} \varphi_{0}^{(+)}\right)^{2}+\left(\partial_{x} \varphi_{0}^{(-)}\right)^{2}\right) \\
& +\partial_{x} R_{0}^{(+)} \partial_{x} R_{0}^{(-)} \\
& +2\left(\left(R_{0}^{(+)}\right)^{2}+\left(R_{0}^{(-)}\right)^{2}\right)\left[\left.\frac{\partial V}{\partial R}\right|_{\varepsilon=0}\right]^{(-)}+4 R_{0}^{(+)} R_{0}^{(-)}\left[\left.\frac{\partial V}{\partial R}\right|_{\varepsilon=0}\right]^{(+)} .
\end{aligned}
$$

As we have shown in section [5.1, and in particular in sub-section 5.1.1, the mirror timesymmetry property of the charges, given in (5.1.52), is valid for solutions for which the components of the fields with different eigenvalues of $P$ are not mixed. Since, we have two fields $R$ and $\varphi$ we have four possibilities for non-mixing solutions. In our analysis we shall assume that the potentials satisfy the property

$$
\left.\frac{\partial V}{\partial R}\right|_{\varepsilon=0} \sim R_{0}
$$

If one considers solutions for which $R_{0}^{(+)}=0$ and $\partial \varphi_{0}^{(+)}=0$ (with $\partial$ standing for time and space derivatives), then the zero order equations of motion (5.2.9)-(5.2.12) impliy that $R_{0}^{(-)}=0$. In addition, if one assumes $R_{0}^{(+)}=0$ and $\partial \varphi_{0}^{(-)}=0$ then (5.2.9)-(5.2.12) imply $\partial_{t} R_{0}^{(-)}=0$. Finally, if one assumes $R_{0}^{(-)}=0$ and $\partial \varphi_{0}^{(-)}=0$ then one finds that $\partial_{t} R_{0}^{(+)}=0$ and $\partial_{t} \varphi_{0}^{(+)}=0$. Therefore, in none of those three cases one should expect to get interesting solutions, specially two-soliton solutions. Therefore, we shall restrict our attention to the class 
of solutions for which $R_{0}^{(-)}=0$ and $\partial_{t, x} \varphi_{0}^{(+)}=0$, i.e. those that satisfy

$$
P: \quad R_{0} \rightarrow R_{0} \quad \varphi_{0} \rightarrow-\varphi_{0}+\text { const. }
$$

Note that with $R_{0}$ even under $P$ it follows that all derivatives of the form $\left.\frac{\partial^{n+m} V}{\partial \varepsilon^{n} \partial R^{m}}\right|_{\varepsilon=0}$ are even under $P$. Now, assuming (5.2.14) one gets that the first order part of the equations of motion (5.2.1) split under $P$ as

$$
\begin{aligned}
\partial_{t} R_{1}^{(-)} & =-\partial_{x}\left(R_{0}^{(+)} \partial_{x} \varphi_{1}^{(+)}+R_{1}^{(-)} \partial_{x} \varphi_{0}^{(-)}\right), \\
\partial_{t} R_{1}^{(+)} & =-\partial_{x}\left(R_{0}^{(+)} \partial_{x} \varphi_{1}^{(-)}+R_{1}^{(+)} \partial_{x} \varphi_{0}^{(-)}\right), \\
-\left(R_{0}^{(+)}\right)^{2} \partial_{t} \varphi_{1}^{(-)} & =2 R_{0}^{(+)} R_{1}^{(+)} \partial_{t} \varphi_{0}^{(-)}-R_{0}^{(+)} \partial_{x}^{2} R_{1}^{(+)}-R_{1}^{(+)} \partial_{x}^{2} R_{0}^{(+)} \\
& +R_{0}^{(+)} R_{1}^{(+)}\left(\partial_{x} \varphi_{0}^{(-)}\right)^{2}+\left(R_{0}^{(+)}\right)^{2} \partial_{x} \varphi_{0}^{(-)} \partial_{x} \varphi_{1}^{(-)}+\partial_{x} R_{0}^{(+)} \partial_{x} R_{1}^{(+)} \\
& +\left.4 R_{0}^{(+)} R_{1}^{(+)} \frac{\partial V}{\partial R}\right|_{\varepsilon=0}+2\left(R_{0}^{(+)}\right)^{2}\left[\left.\frac{\partial^{2} V}{\partial \varepsilon \partial R}\right|_{\varepsilon=0}+\left.\frac{\partial^{2} V}{\partial R^{2}}\right|_{\varepsilon=0} R_{1}^{(+)}\right], \\
-\left(R_{0}^{(+)}\right)^{2} \partial_{t} \varphi_{1}^{(+)} & =2 R_{0}^{(+)} R_{1}^{(-)} \partial_{t} \varphi_{0}^{(-)}-R_{0}^{(+)} \partial_{x}^{2} R_{1}^{(-)}-R_{1}^{(-)} \partial_{x}^{2} R_{0}^{(+)} \\
& +R_{0}^{(+)} R_{1}^{(-)}\left(\partial_{x} \varphi_{0}^{(-)}\right)^{2}+\left(R_{0}^{(+)}\right)^{2} \partial_{x} \varphi_{0}^{(-)} \partial_{x} \varphi_{1}^{(+)}+\partial_{x} R_{0}^{(+)} \partial_{x} R_{1}^{(-)} \\
+ & \left.4 R_{0}^{(+)} R_{1}^{(-)} \frac{\partial V}{\partial R}\right|_{\varepsilon=0}+\left.2\left(R_{0}^{(+)}\right)^{2} \frac{\partial^{2} V}{\partial R^{2}}\right|_{\varepsilon=0} R_{1}^{(-)} .
\end{aligned}
$$

Once the zero order solutions for $R_{0}^{(+)}$and $\varphi_{0}^{(-)}$have been found, we put them into (5.2.15)(5.2.18) and get four coupled partial differential equations with non-constant coefficients which are linear in the first order fields $R_{1}^{( \pm)}$and $\varphi_{1}^{( \pm)}$.

There are two important facts about (5.2.15)-(5.2.18). First they couple $R_{1}^{(+)}$only to $\varphi_{1}^{(-)}$and $R_{1}^{(-)}$only to $\varphi_{1}^{(+)}$, i.e. the pair of equations (5.2.15) and (5.2.18) is decoupled from the pair formed by (5.2.16) and (5.2.17). Secondly, the pair of equations (5.2.15) and (5.2.18) is homogeneous in the first order fields, but the equation (5.2.17) is non-homogeneous due to the term $\left.2\left(R_{0}^{(+)}\right)^{2} \frac{\partial^{2} V}{\partial \varepsilon \partial R}\right|_{\varepsilon=0}$, which does not involve the first order fields. Therefore, there are no solutions for which $R_{1}^{(+)}=0$ and $\varphi_{1}^{(-)}=$constant. On the other hand we can have solutions for which $R_{1}^{(-)}=0$ and $\varphi_{1}^{(+)}=$constant. In addition, if $R_{1}$ and $\varphi_{1}$, are solutions with a non-definite parity, then $R_{1}-R_{1}^{(-)}$and $\varphi_{1}-\varphi_{1}^{(+)}$are also solutions but now with a definite parity. So, we can always choose the first order solutions to satisfy

$$
P: \quad R_{1} \rightarrow R_{1} \quad \varphi_{1} \rightarrow-\varphi_{1}+\text { const. }
$$

If we now take the zero and first order solutions satisfying (5.2.14) and (5.2.19), respec- 
tively, then the second order part of the equations of motion (5.2.1) splits under $P$ as

$$
\begin{aligned}
& \partial_{t} R_{2}^{(-)}=-\partial_{x}\left(R_{2}^{(-)} \partial_{x} \varphi_{0}^{(-)}\right)-\partial_{x}\left(R_{0}^{(+)} \partial_{x} \varphi_{2}^{(+)}\right) \\
& \partial_{t} R_{2}^{(+)}=-\partial_{x}\left(R_{2}^{(+)} \partial_{x} \varphi_{0}^{(-)}\right)-\partial_{x}\left(R_{0}^{(+)} \partial_{x} \varphi_{2}^{(-)}\right)-\partial_{x}\left(R_{1}^{(+)} \partial_{x} \varphi_{1}^{(-)}\right)
\end{aligned}
$$

and

$$
\begin{aligned}
& -\left(R_{0}^{(+)}\right)^{2} \partial_{t} \varphi_{2}^{(-)}-2 R_{0}^{(+)} R_{1}^{(+)} \partial_{t} \varphi_{1}^{(-)}-\left(\left(R_{1}^{(+)}\right)^{2}+2 R_{0}^{(+)} R_{2}^{(+)}\right) \partial_{t} \varphi_{0}^{(-)} \\
& =-R_{0}^{(+)} \partial_{x}^{2} R_{2}^{(+)}-R_{2}^{(+)} \partial_{x}^{2} R_{0}^{(+)}-R_{1}^{(+)} \partial_{x}^{2} R_{1}^{(+)} \\
& +\left(R_{0}^{(+)}\right)^{2} \partial_{x} \varphi_{0}^{(-)} \partial_{x} \varphi_{2}^{(-)}+\frac{1}{2}\left(R_{0}^{(+)}\right)^{2}\left(\partial_{x} \varphi_{1}^{(-)}\right)^{2}+2 R_{0}^{(+)} R_{1}^{(+)} \partial_{x} \varphi_{0}^{(-)} \partial_{x} \varphi_{1}^{(-)} \\
& +R_{0}^{(+)} R_{2}^{(+)}\left(\partial_{x} \varphi_{0}^{(-)}\right)^{2}+\frac{1}{2}\left(R_{1}^{(+)}\right)^{2}\left(\partial_{x} \varphi_{0}^{(-)}\right)^{2} \\
& +\frac{1}{2}\left(\partial_{x} R_{1}^{(+)}\right)^{2}+\partial_{x} R_{0}^{(+)} \partial_{x} R_{2}^{(+)}+\left.\left[2\left(R_{1}^{(+)}\right)^{2}+4 R_{0}^{(+)} R_{2}^{(+)}\right] \frac{\partial V}{\partial R}\right|_{\varepsilon=0} \\
& +4 R_{0}^{(+)} R_{1}^{(+)}\left[\left.\frac{\partial^{2} V}{\partial \varepsilon \partial R}\right|_{\varepsilon=0}+\left.\frac{\partial^{2} V}{\partial R^{2}}\right|_{\varepsilon=0} R_{1}^{(+)}\right] \\
& +\left(R_{0}^{(+)}\right)^{2}\left[\left.\frac{\partial^{3} V}{\partial \varepsilon^{2} \partial R}\right|_{\varepsilon=0}+\left.2 \frac{\partial^{3} V}{\partial \varepsilon \partial R^{2}}\right|_{\varepsilon=0} R_{1}^{(+)}+\left.\frac{\partial^{3} V}{\partial R^{3}}\right|_{\varepsilon=0}\left(R_{1}^{(+)}\right)^{2}+\left.2 \frac{\partial^{2} V}{\partial R^{2}}\right|_{\varepsilon=0} R_{2}^{(+)}\right]
\end{aligned}
$$

and

$$
\begin{aligned}
& -\left(R_{0}^{(+)}\right)^{2} \partial_{t} \varphi_{2}^{(+)}-2 R_{0}^{(+)} R_{2}^{(-)} \partial_{t} \varphi_{0}^{(-)}=-R_{0}^{(+)} \partial_{x}^{2} R_{2}^{(-)}-R_{2}^{(-)} \partial_{x}^{2} R_{0}^{(+)} \\
& +\left(R_{0}^{(+)}\right)^{2} \partial_{x} \varphi_{0}^{(-)} \partial_{x} \varphi_{2}^{(+)}+R_{0}^{(+)} R_{2}^{(-)}\left(\partial_{x} \varphi_{0}^{(-)}\right)^{2} \\
& +\partial_{x} R_{0}^{(+)} \partial_{x} R_{2}^{(-)}+\left.4 R_{0}^{(+)} R_{2}^{(-)} \frac{\partial V}{\partial R}\right|_{\varepsilon=0} \\
& +\left.2 \frac{\partial^{2} V}{\partial R^{2}}\right|_{\varepsilon=0} R_{2}^{(-)}
\end{aligned}
$$

Again we have a structure very similar to that discussed in the case of the equations (5.2.15)-(5.2.18). Indeed, having found the solutions for the zero and first order fields, we put them into (5.2.20)-(5.2.23) and get four coupled partial differential equations with nonconstant coefficients which are linear in $R_{2}^{( \pm)}$and $\varphi_{2}^{( \pm)}$. In addition, the pair of equations (5.2.20) and (5.2.23) is decoupled from the pair (5.2.21) and (5.2.22), i.e. $R_{2}^{(+)}$couples only to $\varphi_{2}^{(-)}$and $R_{2}^{(-)}$also only to $\varphi_{2}^{(+)}$. Again, the pair of equations (5.2.20) and (5.2.23) is homogeneous in the second order fields and the pair (5.2.21) and (5.2.22) is non-homogeneous. Thus, as before, $R_{2}^{(-)}=0$ and $\varphi_{2}^{(+)}=$constant is a solution, but $R_{2}^{(+)}=0$ and $\varphi_{2}^{(-)}=$ constant, cannot be a solution. In addition, if $R_{2}$ and $\varphi_{2}$, are solutions, with a non-definite parity, then $R_{2}-R_{2}^{(-)}$and $\varphi_{2}-\varphi_{2}^{(+)}$are also solutions but now with a definite parity. So, we 
can always choose the second order solutions to satisfy

$$
P: \quad R_{2} \rightarrow R_{2} \quad \varphi_{2} \rightarrow-\varphi_{2}+\text { const. }
$$

We can repeat this process, and even though we have not proved this here, this structure repeats itself at every order of perturbation in $\varepsilon$. Therefore, the fields $R^{(-)}$and $\varphi^{(+)}$can always be "gauged away" since they satisfy homogeneous equations (order by order), but the fields $R^{(+)}$and $\varphi^{(-)}$are robust in the sense that they always have to be present in the solution. Thus, we have shown that the solutions satisfying (5.1.38) are favoured by the dynamics when the potential $V$ in (5.1.1) is a deformation of the integral NLS potential (5.1.9). We point out however, that the property of being even or odd under $P$ is not something that can be encoded into the initial boundary conditions at a given initial time $t_{0}$. The properties under $P$ involve a link between the past and the future of the solution and so, perhaps, cannot be understood using the usual techniques (specially numerical) of investigating the coupling of the normal modes as the systems evolves in time. We are perhaps facing a new and intriguing non-liner phenomenon. In the next section, we go further in our analysis and study the properties under $P$ of the exact one and two-soliton solutions of the integral NLS theory.

\subsection{The parity properties of NLS solitons}

We will now analyze the one and two soliton solutions of the integrable NLS theory (5.1.10) under the parity transformation (5.1.37). The solutions are constructed by the Hirota's method described in the appendix C.

\subsubsection{The one-soliton solutions}

In terms of the fields $R$ and $\varphi$ introduced in (15.1.18) the one-bright-soliton solution (5.1.11) is given by

$$
R_{0}^{\text {bright }}=\frac{\rho^{2}}{|\eta|} \frac{1}{\cosh ^{2}\left[\rho\left(x-v t-x_{0}\right)\right]} ; \quad \varphi_{0}^{\text {bright }}=2\left[\left(\rho^{2}-\frac{v^{2}}{4}\right) t+\frac{v}{2} x\right]
$$

Analogously, the one-dark-soliton solution (5.1.12) is given by

$$
R_{0}^{\mathrm{dark}}=\frac{\rho^{2}}{\eta} \tanh ^{2}\left[\rho\left(x-v t-x_{0}\right)\right] ; \quad \varphi_{0}^{\mathrm{dark}}=2\left[\frac{v}{2} x-\left(2 \rho^{2}+\frac{v^{2}}{4}\right) t\right]
$$


Then it is clear that the relevant parity transformation, in each case, is

$$
P: \quad \tilde{x} \rightarrow-\tilde{x} \quad t \rightarrow-t \quad \text { with } \quad \tilde{x}=x-x_{0}
$$

Therefore one has that

$$
P: \quad R_{0}^{\text {bright/dark }} \rightarrow R_{0}^{\text {bright/dark }} ; \quad \varphi_{0}^{\text {bright/dark }} \rightarrow-\varphi_{0}^{\text {bright/dark }}+2 v x_{0}
$$

which is agreement with (5.2.14) and (5.1.38).

If one chooses the potential in (5.1.1) as

$$
V=\frac{2}{2+\varepsilon} \eta R^{2+\varepsilon}
$$

then the theory has a one-soliton solution given by

$$
R=\left[\frac{2+\varepsilon}{2} \frac{\rho^{2}}{|\eta|} \frac{1}{\cosh ^{2}\left[(1+\varepsilon) \rho\left(x-v t-x_{0}\right)\right]}\right]^{\frac{1}{1+\varepsilon}} ; \quad \varphi=2\left[\left(\rho^{2}-\frac{v^{2}}{4}\right) t+\frac{v}{2} x\right]
$$

which is a deformation of the one-bright-soliton (5.3.1). Notice that under the parity (5.3.3) it transforms as

$$
P: \quad R \rightarrow R ; \quad \varphi \rightarrow-\varphi+2 v x_{0}
$$

Since (5.3.6) is an exact solution of the deformed NLS theory this observation supports our claims of section 5.2. based on the perturbative series in $\varepsilon$, that solutions satisfying the property (5.3.7) are favoured by the dynamics.

\subsubsection{The two-soliton solutions}

The two-bright-soliton solution of the NLS model can been obtained using the Hirota method. The details are given in the appendix [C. Its expression is given in (C.0.13), which can be rewritten as

$$
\psi_{0}=\frac{2}{\sqrt{|\eta|}} \frac{\mathcal{N}}{\mathcal{D}}
$$

where the overall phase $i e^{-i \phi}$, has been absorbed using the symmetry (5.1.8), and where we have defined

$$
\mathcal{D}=2 e^{z_{+}}\left[\cosh z_{+}+e^{-\Delta} \cosh z_{-}-16 \frac{\left|\rho_{1}\right|\left|\rho_{2}\right|}{\Lambda_{-}} \cos \left(\Omega_{1}-\Omega_{2}-2 \delta_{+}\right)\right]
$$


and

$$
\begin{aligned}
\mathcal{N} & =e^{z_{+}} e^{-\frac{\Delta}{2}} e^{-i \frac{\left(\Omega_{1}+\Omega_{2}\right)}{2}} e^{-i \delta_{-}}\left[e^{-\frac{z_{+}}{2}} e^{i \delta_{-}}\left(\left|\rho_{1}\right| e^{-i \frac{\left(\Omega_{1}-\Omega_{2}\right)}{2}} e^{\frac{z_{-}}{2}}+\left|\rho_{2}\right| e^{i \frac{\left(\Omega_{1}-\Omega_{2}\right)}{2}} e^{-\frac{z_{-}}{2}}\right)\right. \\
& \left.+e^{\frac{z_{+}}{2}} e^{-i \delta_{-}}\left(\left|\rho_{2}\right| e^{i \frac{\left(\Omega_{1}-\Omega_{2}\right)}{2}} e^{\frac{z_{-}}{2}} e^{-i 2 \delta_{+}}+\left|\rho_{1}\right| e^{-i \frac{\left(\Omega_{1}-\Omega_{2}\right)}{2}} e^{-\frac{z_{-}}{2}} e^{i 2 \delta_{+}}\right)\right]
\end{aligned}
$$

In this expression we use $\Delta$ defined by

$$
e^{\Delta}=\frac{\Lambda_{-}}{\Lambda_{+}}=\frac{\left(v_{1}-v_{2}\right)^{2}+4\left(\rho_{1}-\rho_{2}\right)^{2}}{\left(v_{1}-v_{2}\right)^{2}+4\left(\rho_{1}+\rho_{2}\right)^{2}}
$$

and the coordinates

$$
z_{+} \equiv X_{1}+X_{2}+\Delta \quad z_{-} \equiv X_{1}-X_{2}
$$

with

$$
X_{i}=\rho_{i}\left(x-v_{i} t-x_{i}^{(0)}\right) \quad \Omega_{i}=\left(\frac{v_{i}^{2}}{4}-\rho_{i}^{2}\right) t-\frac{v_{i}}{2} x+\theta_{i}+\zeta_{i} \quad i=1,2
$$

where

$$
\delta_{ \pm}=\operatorname{ArcTan}\left[\frac{2\left(\rho_{1} \pm \rho_{2}\right)}{\left(v_{1}-v_{2}\right)}\right] .
$$

The quantities $\Omega_{i}$ are linear in $x$ and $t$, and so in the new coordinates $z_{ \pm}$. We can therefore, separate the homogeneous dependence on $z_{ \pm}$by writing

$$
\begin{aligned}
& \frac{\Omega_{1}-\Omega_{2}}{2}-\delta_{+}=\Omega_{-}+c, \\
& \frac{\Omega_{1}+\Omega_{2}}{2}+\delta_{-}=\Omega_{+}+d,
\end{aligned}
$$

where $\Omega_{ \pm}$are homogeneous in $z_{ \pm}$, i.e. $\Omega_{ \pm}=\beta_{ \pm}^{+} z_{+}+\beta_{ \pm}^{-} z_{-}$, with $\beta_{ \pm}^{ \pm}$being some constants depending on $v_{i}$ and $\rho_{i}, i=1,2$. Note that the constants $\zeta_{i}$ appearing in the expression of $\Omega_{i}$ in (5.3.13) are the phases of $z_{1}$ and $w_{1}$ given in (C.0.10), and so depend on $v_{i}$ and $\rho_{i}$, $i=1,2$. However, the constants $\theta_{i}$ also appearing in (5.3.13) are the phases of $a_{+}$and $b_{+}$ given in (C.0.9), and so are independent of $v_{i}$ and $\rho_{i}$. The constants $c$ and $d$ introduced in (5.3.16) depend on $v_{i}, \rho_{i}$ and $x_{i}^{(0)}(i=1,2)$ but are linear in $\theta_{1}-\theta_{2}$ and $\theta_{1}+\theta_{2}$, respectively, and so can be traded for $\theta_{i}, i=1,2$, and be considered as constants independent of $v_{i}, \rho_{i}$ and $x_{i}^{(0)}$. Thus, the two-soliton solution (5.3.8) depends only on 8 free parameters; namely, $v_{i}, \rho_{i}, x_{i}^{(0)}(i=1,2) c$ and $d$, and can be written as

$$
\psi_{0}=\frac{e^{-\frac{\Delta}{2}}}{\sqrt{|\eta|}} e^{-i \Omega_{+}} \frac{\hat{\mathcal{N}}}{\hat{\mathcal{D}}}
$$


where the overall phase $e^{-i d}$, has been absorbed using the symmetry (5.1.8), and where we have introduced

$$
\hat{\mathcal{D}}=\cosh z_{+}+e^{-\Delta} \cosh z_{-}-16 \frac{\left|\rho_{1}\right|\left|\rho_{2}\right|}{\Lambda_{-}} \cos \left[2\left(\Omega_{-}+c\right)\right]
$$

and

$$
\begin{aligned}
\hat{\mathcal{N}} & =e^{-\frac{z_{+}}{2}} e^{i \delta_{-}}\left(\left|\rho_{1}\right| e^{-i\left(\Omega_{-}+c+\delta_{+}\right)} e^{\frac{z_{-}}{2}}+\left|\rho_{2}\right| e^{i\left(\Omega_{-}+c+\delta_{+}\right)} e^{-\frac{z_{-}}{2}}\right) \\
& +e^{\frac{z_{+}}{2}} e^{-i \delta_{-}}\left(\left|\rho_{1}\right| e^{-i\left(\Omega_{-}+c-\delta_{+}\right)} e^{-\frac{z_{-}}{2}}+\left|\rho_{2}\right| e^{i\left(\Omega_{-}+c-\delta_{+}\right)} e^{\frac{z_{-}}{2}}\right)
\end{aligned}
$$

We are now in a position to consider the parity transformation (5.1.37) relevant for the two-bright-soliton solution, i.e.

$$
P: \quad\left(z_{+}, z_{-}\right) \rightarrow\left(-z_{+},-z_{-}\right)
$$

which can be written in terms of $x$ and $t$ as

$$
P: \quad(\tilde{x}, \tilde{t}) \rightarrow(-\tilde{x},-\tilde{t}) \quad \text { with } \quad \tilde{x}=x-x_{\Delta} \quad \tilde{t}=t-t_{\Delta}
$$

and

$$
\begin{aligned}
& x_{\Delta}=\frac{\Delta\left(\rho_{1} v_{1}-\rho_{2} v_{2}\right)+2 \rho_{1} \rho_{2}\left(v_{2} x_{1}^{(0)}-v_{1} x_{2}^{(0)}\right)}{\left(2 \rho_{1} \rho_{2}\left(v_{2}-v_{1}\right)\right)} \\
& t_{\Delta}=\frac{\Delta\left(\rho_{1}-\rho_{2}\right)+2 \rho_{1} \rho_{2}\left(x_{1}^{(0)}-x_{2}^{(0)}\right)}{\left(2 \rho_{1} \rho_{2}\left(v_{2}-v_{1}\right)\right)}
\end{aligned}
$$

Note that under this parity transformation $\Omega_{ \pm}$are odd since they are linear and homogeneous in $z_{ \pm}$. Therefore, if

$$
c=n \frac{\pi}{2}, \quad n \in \mathbb{Z}
$$

the term $\cos \left[2\left(\Omega_{-}+c\right)\right]$, in $\hat{\mathcal{D}}$, given in (5.3.18), is invariant under the parity $P$. Consequently, $\hat{\mathcal{D}}$ is even under $P$

$$
P(\hat{\mathcal{D}})=\hat{\mathcal{D}}
$$

In addition, when $c$ satisfies (5.3.23), as one can check,

$$
P(\hat{\mathcal{N}})=(-1)^{n} \hat{\mathcal{N}}^{*}
$$

Thus, the two-bright-soliton solution (5.3.17) satisfies

$$
P\left(\psi_{0}\right)=(-1)^{n} \psi_{0}^{*}
$$




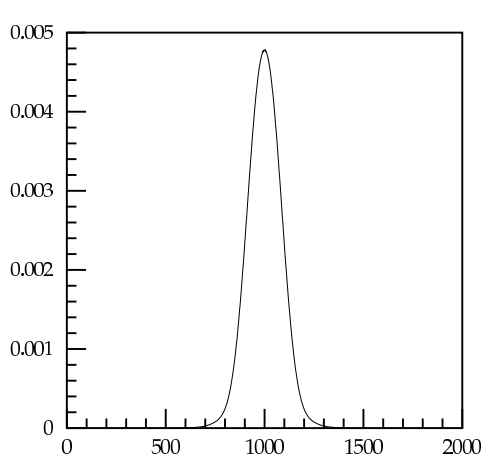

Figure 5.1 - Plot of $|\psi|^{2}$ against $x$ for the one-soliton solution of the unperturbed NLS model.

In terms of the fields $R$ and $\varphi$ introduced in (5.1.18), i.e. for $\psi_{0}=\sqrt{R_{0}} e^{i \frac{\varphi_{0}}{2}}$, we find that

$$
P: \quad R_{0} \rightarrow R_{0} ; \quad \varphi_{0} \rightarrow-\varphi_{0}+2 \pi n
$$

which is what we have assumed in (5.2.14).

\subsection{Numerical analysis}

In this section we present some numerical results which support the claims we have made in the preceding sections.

Our results concern the NLS model and its deformation discussed in the last section i.e. with the potential of the form (5.3.5). In our numerical studies we used a fixed lattice of 5001 points with time evolution calculated using the 4th order Runge-Kutta method. The lattice step was taken to be $d x=0.01$ (and sometimes 0.05 or 0.1 ) and the time step used was $\mathrm{dt}=0.00005$. We used both fixed and absorbing boundary conditions (to avoid any reflections from the boundaries) but as our field configurations were always very localised in the main section of the lattice the results did not depend on the boundary conditions (we only considered the evolution of the solitons when they were still some distance away from these boundaries.

\subsubsection{The NLS model}

Let us first present some of our results for the NLS model i.e., for $\varepsilon=0$ ). The one soliton solution, (5.1.11), for the case of $v=0$, is shown in figure (5.1). In this figure we present a plot of $|\psi|^{2}$ as a function of $x$.

Next we have looked at several field configurations involving two solitons i.e., given by (5.3.17). In this case we varied the values of the free parameter $c$. As mentioned in the last 
section, when $c$ is an integer multiple of $\frac{\pi}{2}$ the two-soliton field configuration (5.3.17) is an eigenfunction of $P$ in the sense of (5.3.27). We have followed the field configuration given by (5.3.17) and have used this field configuration as an initial condition for a full simulation and the results were virtually indistinguishable from each other. This has provided a test of our numerical procedure. In figure (5.2) ( $a, b$ and $c$ ) we present plots of the position of one soliton as a function of time for 3 different values of $c$, namely $c=0, c=0.7$ and $c=1.4$. The position was determined by looking at the maxima of the energy density. The trajectory of the other soliton is symmetric to this one and to the right. We notice a slight dependence on the values of $c$.

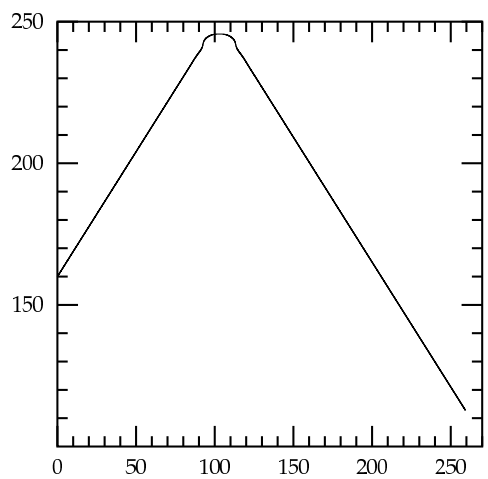

(a) $c=0$

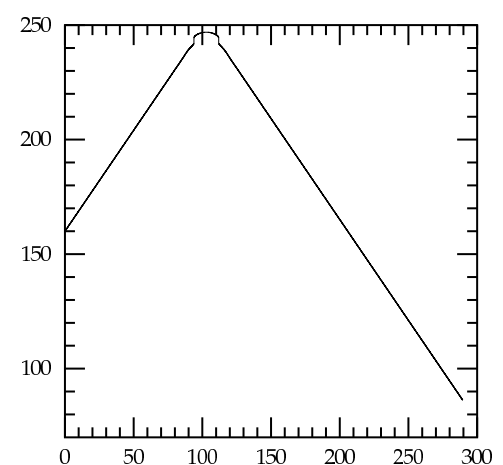

(b) $\mathrm{c}=0.7$

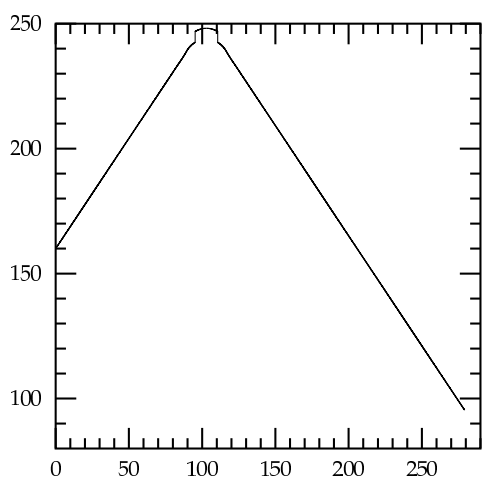

(c) $\mathrm{c}=1.4$

Figure $5.2-\quad$ Trajectories of two Solitons at $v=0.4(\epsilon=0)$

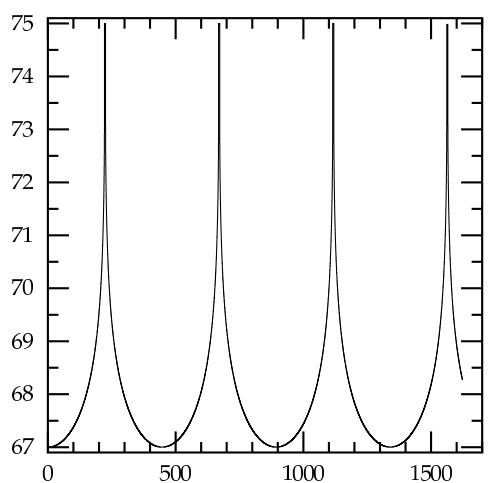

(a) $\mathrm{c}=0$

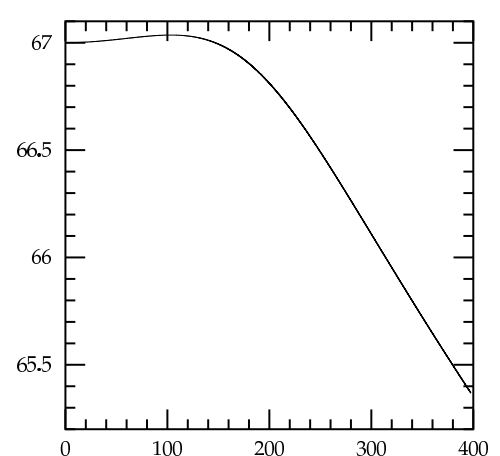

(b) $\mathrm{c}=0.7$

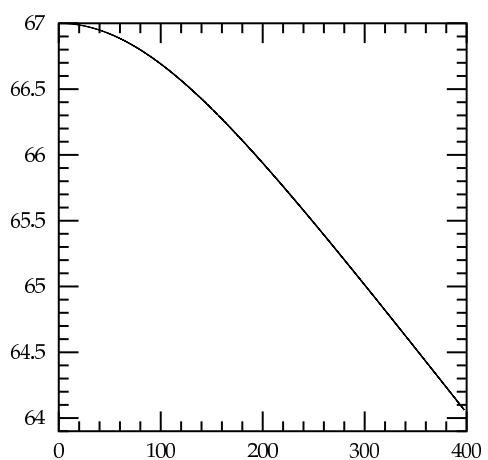

(c) $\mathrm{c}=1.4$

Figure 5.3 - Trajectories of two solitons at rest $(\epsilon=0)$

The existence of multi-soliton solutions does not directly describe the forces between the solitons. Of course, one can deduce them by analysing in detail the time dependence of their positions etc. Another way to proceed involves putting two solitons at rest, not too close (not to deform them) and not too far away (so that they do interact) and see what will happen.

We have performed such a study and in (5.3) we present the trajectories of the soliton "on 


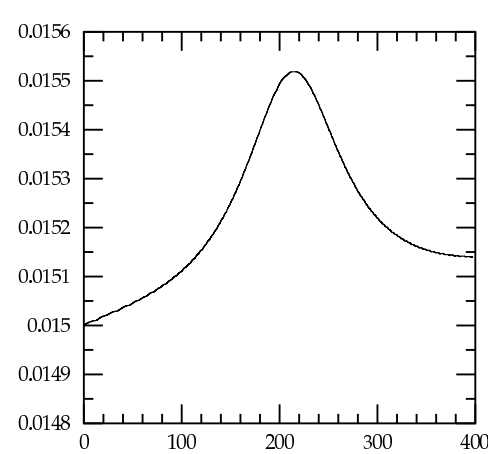

(a) $c=0$

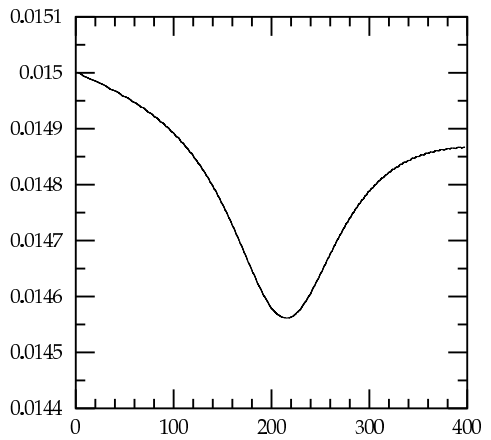

(b) $\mathrm{c}=0.7$

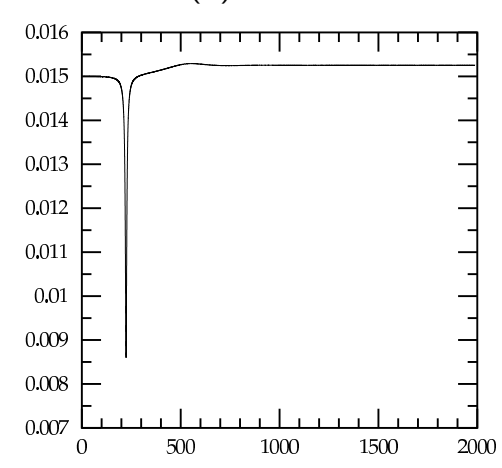

(d) $c=0.01$

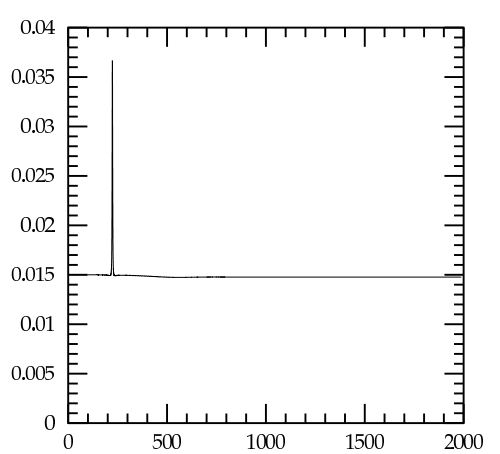

(c) $\mathrm{c}=1.4$ .

.

Figure $5.4-\quad$ Heights of the solitons originaly at rest $(\epsilon=0)$

the left" with respect to time for 3 values of the relative phase between them (equivalent to $c$ ). We see that at $c=0$ the solitons attract, at $c=0.7$ the forces are quite complicated resulting in a rather complicated trajectories and for $c=1.4$ they repel. However, the parameter $c$ has also another role and this is associated with the heights of the solitons. When $c=0$ both solitons, when they move towards each other, stay of the same size but as they come towards each other they overlap and some appear to be taller. When $c \neq 0$ the situation is more complicated. The non-zero value of $c$ breaks the symmetry and so one soliton tends to grow while the other, to decrease in size. For this to happen they have to interact and so be close enough; the two effects (both of them growing and one of them growing and the other one getting smaller) produce a more complicated pattern of their sizes and, in part, is responsible for their repulsion and never being able to come very close to each other. Hence the effect of them overlapping is very small. In figure (5.4). we present the time dependence of the heights of the solitons for the cases of $c=0.3$ and $c=0.01$. The first two pictures (from the left) show the time dependence of the heights of the two solitons for $c=0.3$, and the other two for $c=0.01$. The extremum of height seen in plots $a$ ) and $b$ ) corresponds to the case when the two solitons are at the closest distance from each other. In the plots $c$ ) and $d$ ) we note that after the scattering the values of the heights are slightly different. This may appear strange at first but the two solitons move with marginally different velocities after the scattering; this 
effect is induced during the scattering by the non-zero value of $c$.

And what about the conserved charges? Well, the NLS model is integrable so that all anomalies vanish (and so all charges are conserved). In the next subsection we look at the same problems for $\epsilon \neq 0$ i.e., when the model is not integrable.

\subsubsection{The modified model with $\varepsilon \neq 0$}

Next we have considered the $\varepsilon \neq 0$ cases. This time we have only one soliton solution (5.3.6) which is a simple deformation of the one soliton of the NLS model (5.1.11). In fact, when one plots it for small values of $\varepsilon$ it is hard to see any difference.

As for $\varepsilon \neq 0$ the model is non-integrable and we do not have analytic expressions involving two solitons. Hence we can only use two one solitons some distance apart or use the twosoliton solutions of the NLS model i.e., the expression for $\varepsilon=0$ ) and take them as the initial conditions for our numerical simulations.

In figure (5.5) we present the plots of the trajectories of one soliton (similar to figure (5.2)) for $\varepsilon=0.06$ for 3 values of $c$.

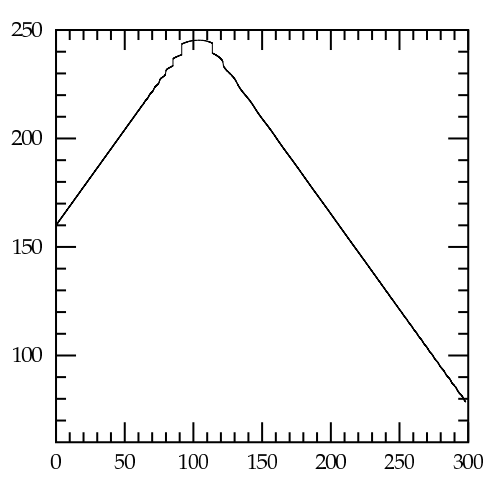

(a) $\mathrm{c}=0$

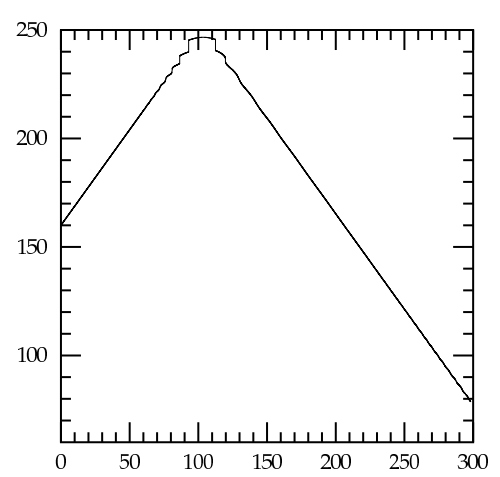

(b) $c=0.7$

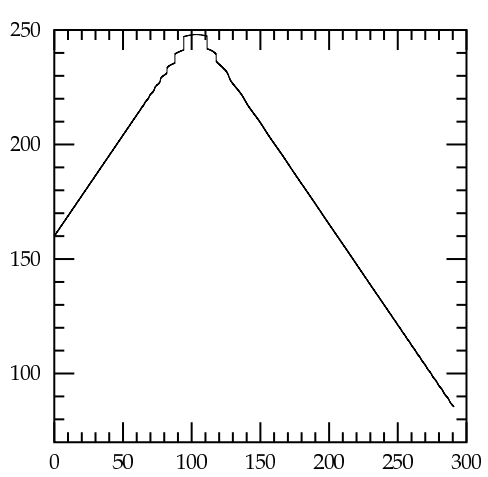

(c) $\mathrm{c}=1.4$

Figure 5.5 - Trajectories of two solitons at $v=0.4(\epsilon=0.06)$

Looking at the trajectories and comparing them to those of the NLS model we see very little difference. The same was observed for other values of $\varepsilon$. In fact these trajectories were obtained by starting with initial configurations corresponding to the NLS model and then evolving them with $\varepsilon \neq 0$. We have also looked at the effects of evolving the initial configurations described by two $\varepsilon \neq 0$ solitons 'sewn' together. The obtained trajectories were very similar. This is due to the fact that the solitons are well localised and all the perturbations induced by taking non-exact expressions were very small.

Next we looked at two solitons at rest. In this case we have taken the expressions for two 
solitons corresponding to $\varepsilon \neq 0$ placed next to each other. In figure (5.6) and (5.7) we present the plots similar to those of figure (15.3) for $\varepsilon=0.06$ and for $\varepsilon=-0.06$.

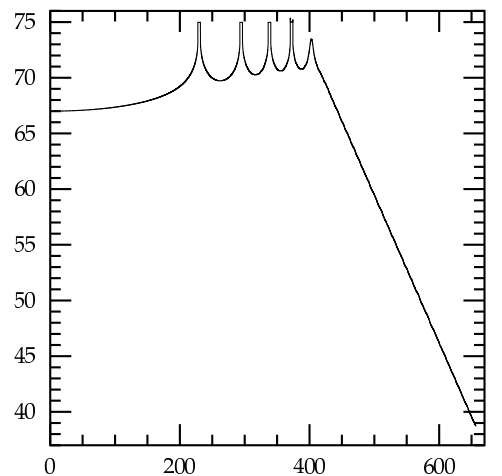

(a) $c=0$.

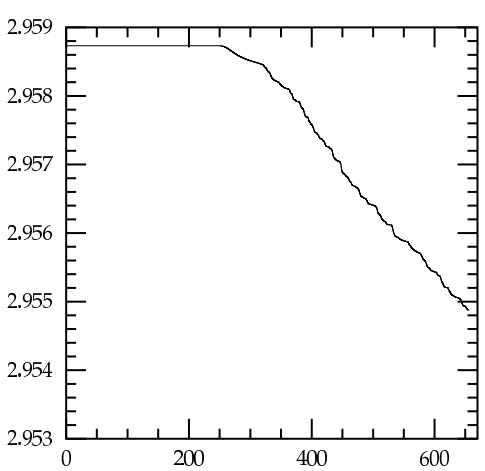

(b) Energy for $\mathrm{c}=0$.

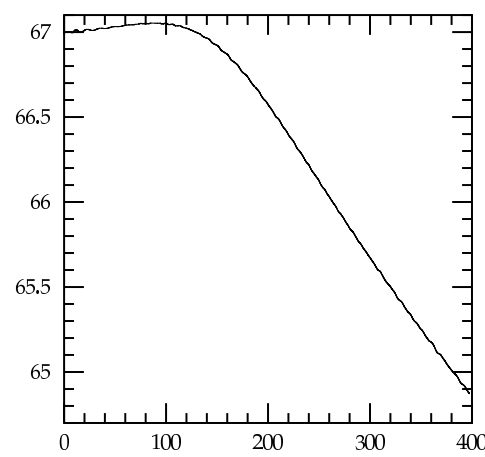

(c) $\mathrm{c}=0.7$.

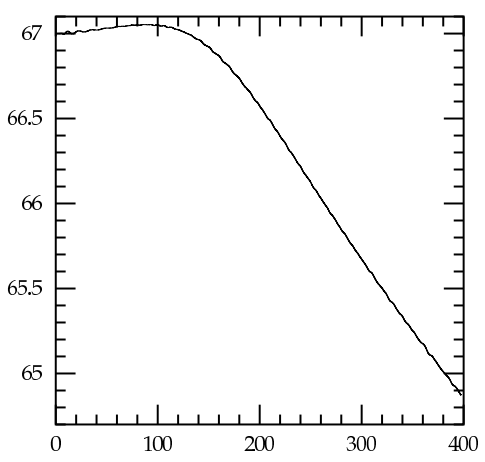

(d) $\mathrm{c}=1.4$.

Figure 5.6 - Trajectories (and the energy) of two solitons at rest $(\epsilon=0.06)$

Comparing these plots with those of figure (15.3) we see only little difference. The dependence on $c$ is very similar although the strength of the attraction (or repulsion) does appear to depend on $\epsilon$. Clearly the overall attraction (at least for $c=0$ ) increases with the increase of $\epsilon$. In addition, we note that for $c=0$, in the NLS case, the solitons oscillate around their point of attraction while for $\epsilon \neq 0$ the amplitude of their oscillation decreases (see figure (5.3(a) and compare with figures (5.6(a) and $(5.7(\mathrm{a}))$ ). This suggests that for $\varepsilon \neq 0$ the solitons radiate a little and so come closer and closer to each other after each oscillation. This is indeed the case as can be seen from the expressions of the total energy (for $c=0$, the energy is effectively conserved while for $c \neq 0$ it decreases a little (see figures $6 b$ ) and $7 b$ ). After a while, however, during these interactions, they gradually change their height and then they split up, repel and move away from each other. During this last part of the motion they move with slightly different velocities and so their sizes are also slightly different. In this their behaviour resembles the $c \neq 0$ case; so we note that as $\varepsilon \neq 0$ the interaction between the solitons gradually induces their behaviour as if $c$ were not 0 . In figure (5.8) we plot the heights of the two solitons observed in the scattering in the $\varepsilon=0.06, c=0$ case. Figure (5.8(a) corresponds to the case of the left hand one, and figure $(5.8(\mathrm{~b}))$ - the right one. 


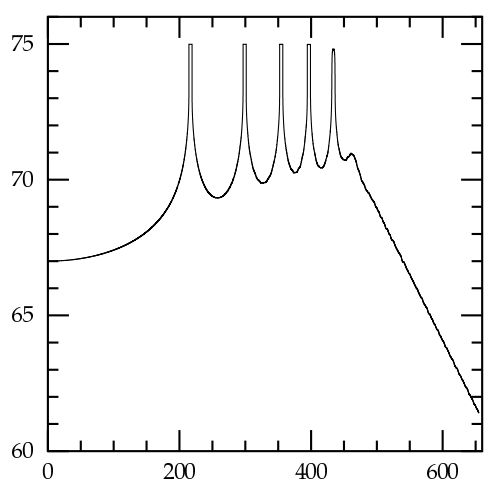

(a) $c=0$.

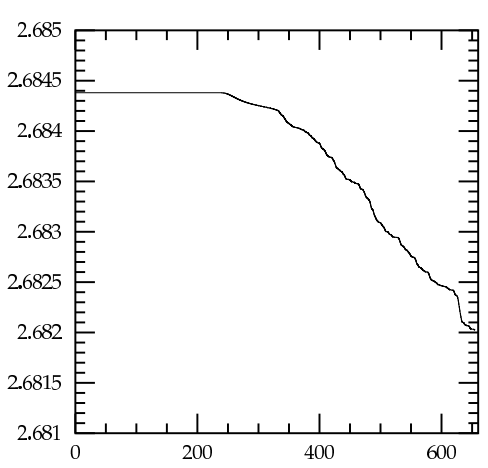

(b) Energy for $\mathrm{c}=0$.

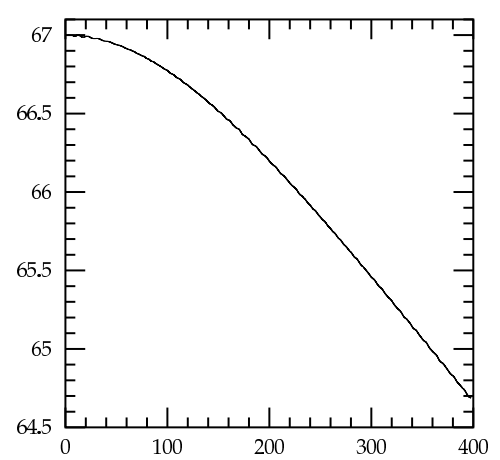

(d) $c=1.4$.

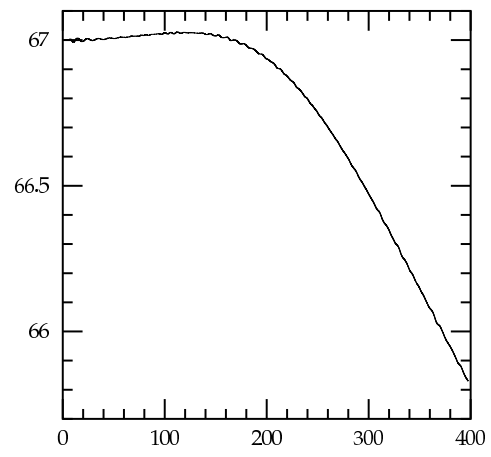

(c) $\mathrm{c}=0.7$.

Figure 5.7 - Trajectories (and the energy) of two solitons at rest $(\epsilon=-0.06)$

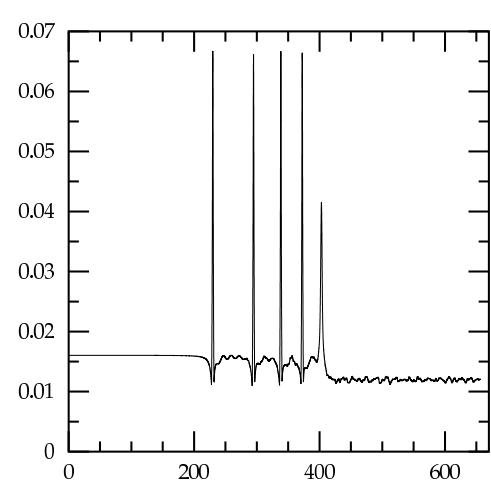

(a) The left one.

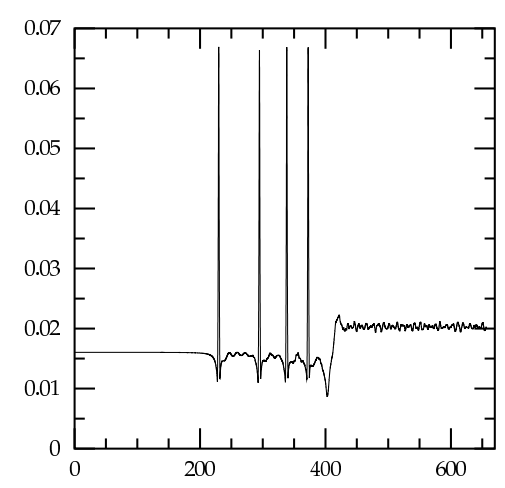

(b) The right one.

Figure 5.8 - Heights of the two solitons observed in their scattering at rest $(\epsilon=-0.06 c=0)$

Furthermore, in the last section we did stress that the cases of $c$ given by (5.3.23) are special for all $\varepsilon$ 's as then we could use our parity arguments to claim asymptotic conservation of further anomalous conserved quantities (5.1.52).

So we have looked at the first nontrivial anomaly. To get its form we used the expression of our potential (5.3.5) and so calculated $X$ from the second formula in (5.1.24). Then we put it into the formula for $\alpha^{(3,-4)}$ given in (B.0.4). In order to avoid using the explicit value of $t_{\Delta}$, which for the zero order solution (expanded in $\varepsilon$ ) is given in (5.3.22), we decided to integrate the resultant expression for $\beta_{4}$. Therefore, using (15.1.24), (5.1.35) and (B.0.4) we 
introduce the quantity

$$
\begin{aligned}
\chi^{(4)}(t) & \equiv \int_{-\infty}^{t} d t^{\prime} \beta_{4}=\int_{-\infty}^{t} d t^{\prime} \int_{-\infty}^{\infty} d x X \alpha^{(3,-4)} \\
& =-2 i \eta^{2} \int_{-\infty}^{t} d t^{\prime} \int_{-\infty}^{\infty} d x\left(R^{\varepsilon}-1\right)\left[6 \eta R^{3}+\frac{3}{2}\left(\partial_{x} \varphi\right)^{2} R^{2}-2 R \partial_{x}^{2} R+\frac{3}{2}\left(\partial_{x} R\right)^{2}\right]
\end{aligned}
$$

As at large values of $t^{\prime}$ the integrand in (5.4.1) vanishes, we can take, in our numerical simulations, the lower end of the $t^{\prime}$-integral to be large in the past but finite. It is the quantity $\chi^{(4)}$ given in (5.4.1) whose plots we present next.

Clearly for $\varepsilon=0$ the anomaly vanishes so in fig 9 and 10 we present our results for $\varepsilon=0.06$ and in fig 11 and 12 those for $\varepsilon=-0.06$.

The first figures in each group show the anomaly when the solitons were sent towards each other at $v=0.4$ and the second ones (10 and 12) those started at rest. In each case the first figure corresponds to the special value of $c$, i.e. $c=0$, the others to $c=0.7$ and $c=1.4$. Note that the scale on the vertical axis in the figures is very different. The anomaly for the cases corresponding to $c=0$ is essentially zero thus supporting our claims of the previous section. Of course, our results are non-perturbative but they do involve also small corrections due to the numerical errors. In any case the smallness of the corrections suggest to us that our claims are correct and the results are stable with respect to small perturbations. For $c \neq 0$ we do see some important corrections to the anomaly as expected (even though the differences of the trajectories are not very significant).

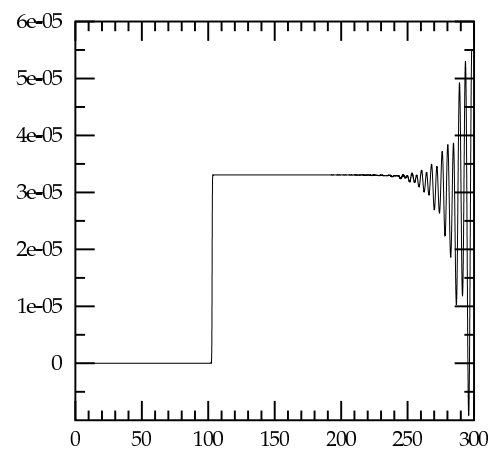

(a) $c=0$.

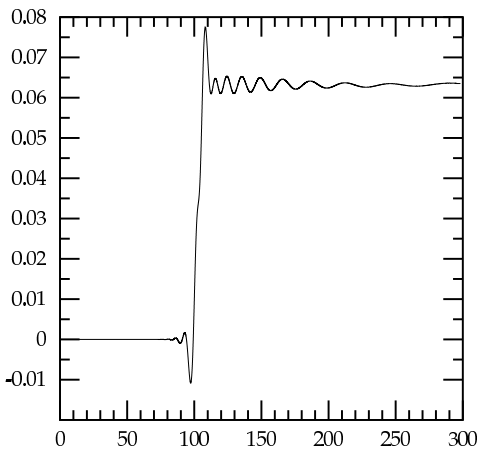

(b) $\mathrm{c}=0.7$.

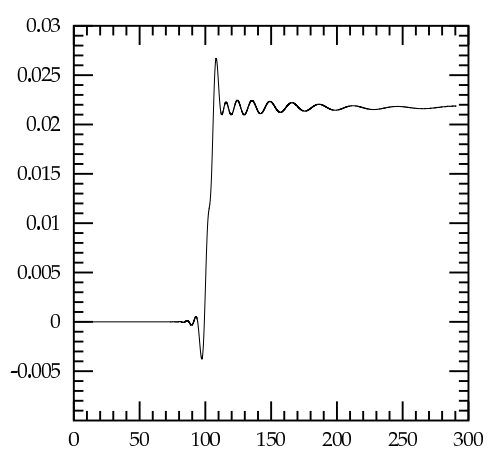

(c) $\mathrm{c}=1.4$.

Figure 5.9 - Time integrated anomaly of two solitons sent at $v=0.4(\epsilon=0.06)$ 


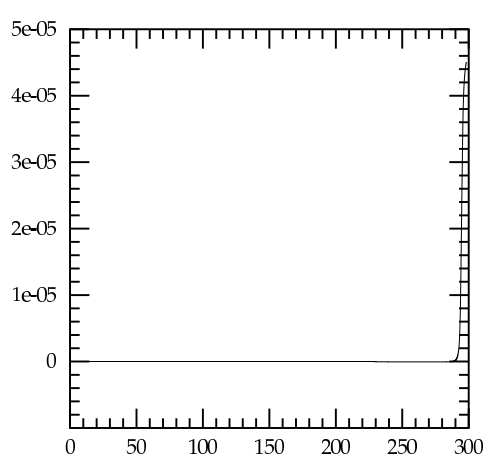

(a) $c=0$.

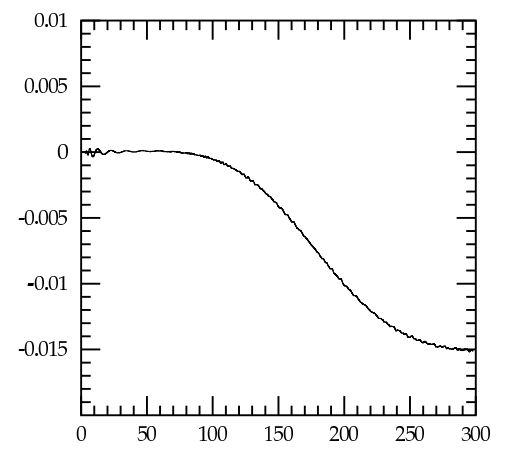

(b) $c=0.7$.

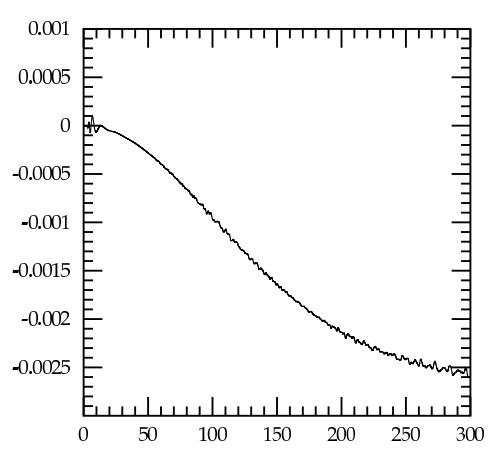

(c) $\mathrm{c}=1.4$.

Figure 5.10 - Time integrated anomaly of two solitons at rest $(\epsilon=0.06)$

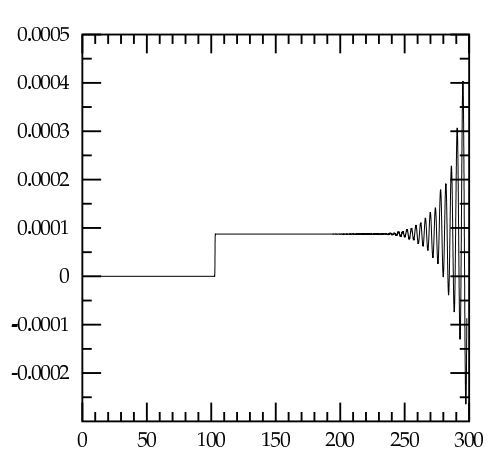

(a) $c=0$.

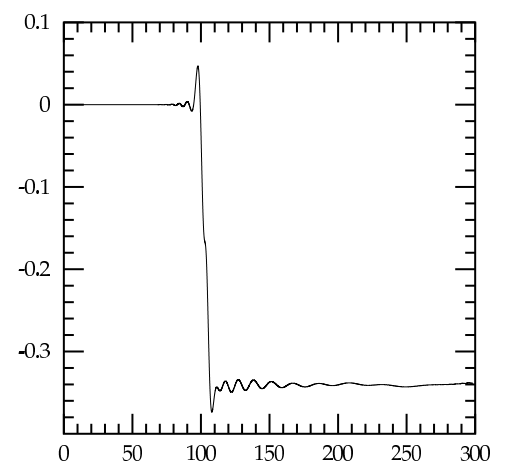

(b) $c=0.7$.

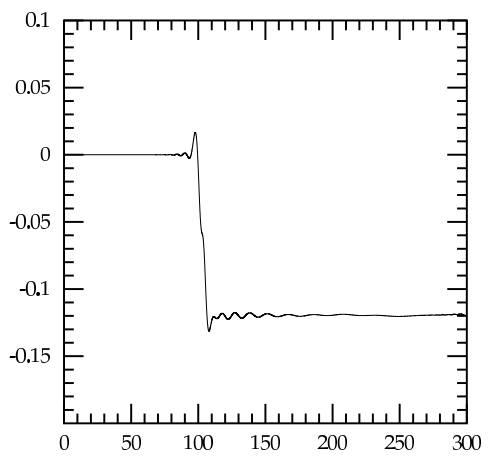

(c) $\mathrm{c}=1.4$.

Figure 5.11 - Time integrated anomaly of two solitons sent at $v=0.4(\epsilon=-0.06)$

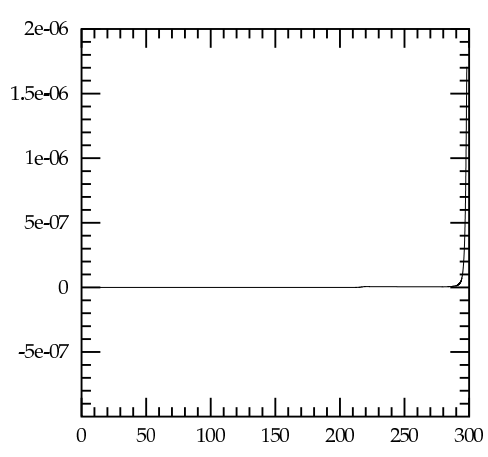

(a) $c=0$.

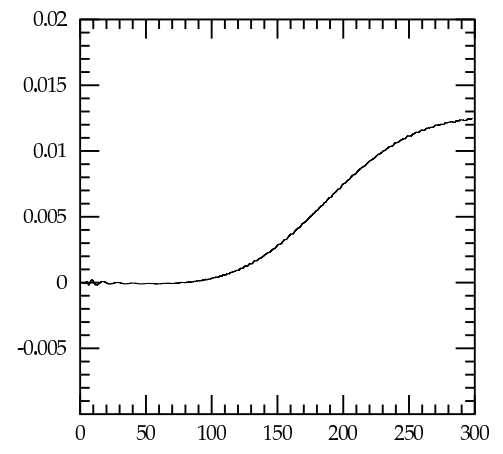

(b) $c=0.7$.

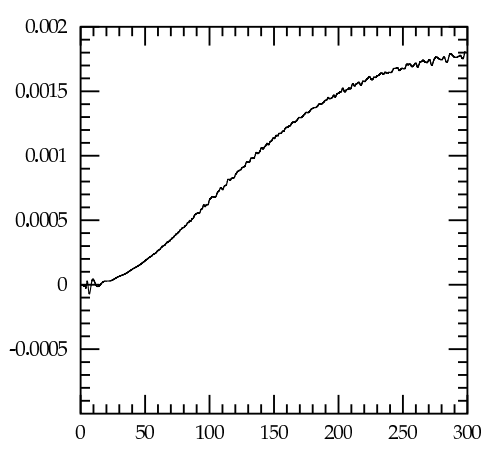

(c) $\mathrm{c}=1.4$.

Figure $5.12-\quad$ Time integrated anomaly of two solitons at rest $(\epsilon=-0.06)$ 


\section{CHAPTER 6}

\section{Final Comments}

This thesis is about using methods and/or ideas from integrable field theories to investigate non-integrable ones. We found that in gauge theories it is possible to construct charges that are not, in principle, related to the Noether's charges, in an analogous way one gets the hidden charges in integrable theories in $(1+1)$-dimensional space-time, in many cases responsible for their integrability and/or existece of solitonic solutions. The path-independence leading to these charges is generalised to what we call surface-independence, volume-independence etc. and this property is obtained from the integral equations we proposed, which are like a flux equation, based on the generalised Stokes theorem introduced for the first time in (6). The charges calculated in this way are invariant under any gauge transformation, which is not the case for the standard charges known for non-Abelian gauge theories in the literature. Moreover, their conservation comes from the integral equation, and not from topology or some other features of particular solutions. In this sense, these charges are very general. As presented in appendix $\mathrm{A}$ we developed a regularisation method of the Wilson lines to deal with the singularities of the gauge connection. This is of major importance for our calculations but also can help to treat this common problem when dealing with holonomies in other cases. Our results open an interesting perspective in non-Abelian gauge theories: we showed how the loop space can be a powerful tool in this context. Certainly a deeper mathematical analysis can bring even more structures to our attention. We calculated explicitly the charges of some configurations of Yang-Mills theory in (3+1)-dimensional space-time, but as we understand, there are a finite number of them. Although the path-independence of the Wilson line that appear in $(1+1)$-dimensional integrable theories was extended, we could not understand yet what would play the role of the spectral parameter. This would lead to the possibility of investigation of integrability of gauge theories. The parameters $\alpha$ and $\beta$ appearing in the integral equations and after that in the charge operators and finally in the charges remain to be understood. So far we do not see how to fix them, and this fixing is crucial for the value of the charge. 
We also discussed here the concept, recently introduced by Luiz Ferreira and Wojciech Zakrzewski, of quasi-integrability in the context of the deformations of the NLS model in $(1+1)$ dimensions. The unperturbed model is fully integrable and possesses multi-soliton solutions. The perturbations destroy integrability but the perturbative models still possess soliton solutions.

We have looked at the problem of quasi-integrability and in this case related it to the properties of specific field configurations (like those describing multi-solitons) under very specific parity transformations. It was showed that when one considers the perturbed models which are not integrable, the models do not possess an infinite number of conserved charges (like the integrable ones do). However, when one restricts the attention to specific field configurations, sometimes it is possible to say more. Namely, when the field configurations satisfy some very specific parity conditions (which are often physical in nature) the extra charges, though not conserved, do satisfy some interesting conditions (given in (5.1.52)). These conditions do restrict the scattering properties of solitons and so provide the basis of our understanding of quasi-integrability. We have also looked at the properties of the soliton field configurations numerically and have found a good support of our claims. 


\section{REFERENCES}

1 LAX, P. Integrals of nonlinear equations of evolution and solitary waves. Communications in Pure and Applied Mathematics, v. 21, n. 5, p. 467-490, 1968.

2 FERREIRA, L. A.; ZAKRZEWSKI, W. J. A simple formula for the conserved charges of soliton theories. Journal of High Energy Physics, , n. 9, Sept 2007.

3 MONTONEN, C.; OLIVE, D. Magnetic monopoles as gauge particles. Physics Letters B, Amsterdam, v. 72, n. 1, p. 117-120, 1977.

4 MANTON, N. S.; SUTCLIFFE, P. Topological solitons. Cambridge, UK: Cambridge University Press, 2004.

5 BABELON, O.; BERNARD, D.; TALON, M. Introduction to classical integrable systems. Cambridge, UK: Cambridge University Press, 2003.

6 ALVAREZ, O.; FERREIRA, L.; GUILLEN, J. A new approach to integrable theories in any dimension. Nuclear Physics B, Amsterdam, v. 529, n. 3, p. 689-736, Oct. 1998.

7 ALVAREZ, O.; FERREIRA, L. A.; SANCHEZ-GUILLEN, J. Integrable theories and loop spaces: fundamentals, applications and new developments. International Journal of Modern Physics A, Singapore, v. 24, n. 10, p. 1825-1888, Apr 202009.

8 FERREIRA, L. A.; LUCHINI, G. Gauge and integrable theories in loop spaces. Nuclear Physics B, Amsterdam, v. 858, n. 2, p. 336-365, May 112012.

9 FERREIRA, L. A. Exact vortex solutions in an extended skyrme-faddeev model. Journal of High energy Physics, , n. 5, May 2009.

10 SCHLIEDER, S. Some remarks on charges and their conservation in a classical $S U(2)$ Yang-Mills theory. Nuovo Cimento A: nuclei particles and fields, v. 63, n. 2, p. 137-154, 1981.

11 ABBOTT, L.; DESER, S. Charge definition in non-abelian gauge-theories. Physics Letters $B$, Amsterdam, v. 116, n. 4, p. 259-263, 1982. 
12 TOD, K. Quasi-local charges in yang-mills theory. Proceedings of the Royal Society of London Series A: mathematical physical and engineering sciences, v. 389, n. 1797, p. 369-377, 1983.

13 CHRUSCIEL, P.; KONDRACKI, W. Some global charges in classical Yang-Mills Theory. Physical Review D, v. 36, n. 6, p. 1874-1881, Sept 1987.

14 FARKAS, R.; SZABADOS, L. B. On quasi-local charges and newman-penrose type quantities in yang-mills theories. Classical and Quantum Gravity, v. 28, n. 14, July 2011.

15 WEINBERG, S. The quantum theory of fields: Modern applications. Cambridge, UK: Cambridge University Press, 1995.

16 MANDELSTAM, S. Quantum electrodynamics without potentials. Annals of Physics, New York, v. 19, n. 1, p. 1-24, 1962.

17 YANG, C. Integral formalism for gauge fields. Physical Review Letters, Woodbury, v. 33, n. 7, p. 445-447, 1974.

18 WU, T.; YANG, C. Concept of non-integrable phase factors and global formulation of gauge fields. Physical Review D, v. 12, n. 12, p. 3845-3857, 1975.

19 POLYAKOV, A. String representations and hidden symmetries for gauge fields. Physics Letters B, Amsterdam, v. 82, n. 2, p. 247-250, 1979.

20 EGUCHI, T.; HOSOTANI, Y. Integrability condition in loop space. Physics Letters $B$, Amsterdam, v. 96, n. 3-4, p. 349-351, 1980.

21 MIGDAL, A. Loop equations and $1 / N$ expansion. Physics Reports, v. 102, n. 4, p. 201-290, 1983.

22 MAKEENKO, Y.; MIGDAL, A. Exact equation for the loop average in multicolor qcd. Physics Letters B, Amsterdam, v. 88, n. 1-2, p. 135-137, 1979.

23 RAJEEV, S. Yang-mills theory on loop space. In: . Editor Fariborz, AH. c2003 ( AIP CONFERENCE PROCEEDINGS, v. 687). p. 41-48.

24 LOLL, R. Loop approaches to gauge field-theories. Theoretical and Mathematical Physics, v. 93, n. 3, p. 1415-1432, Dec 1992.

25 FERREIRA, L. A.; LUCHINI, G. Integral form of yang-mills equations and its gauge invariant conserved charges. Physical Review D, v. 86, n. 8, Oct 2012. 
26 FERREIRA, L. A.; ZAKRZEWSKI, W. J. The concept of quasi-integrability: a concrete example. Journal of High Energy Physics, , n. 5, May 2011.

27 BAZEIA, D.; LOSANO, L.; MALBOUISSON, J. M. C.; MENEZES, R. Classical behavior of deformed sine-gordon models. Physica D - Nonliear Phenomena, v. 237, n. 7, p. 937-946, June 2008.

28 DRINFELD, V. G.; SOKOLOV, V. V. Lie algebras and equations of korteweg-de vries type. Journal of Sovietic Mathematics, v. 30, p. 1975-2036, 1984.

29 OLIVE, D.; TUROK, N. Local conserved-densities and zero-curvature conditions for toda lattice field-theories. Nuclear Physics B, Amsterdam, v. 257, n. 2, p. 277-301, 1985.

30 ARATYN, H.; FERREIRA, L.; GOMES, J.; ZIMERMAN, A. The conserved charges and integrability of the conformal affine toda models. Modern Physics Letters A, Singapore, v. 9, n. 30, p. 2783-2801, Sept 1994.

31 FADDEEV, L. D.; TAKHTAJAN, L. A. Hamiltonian methods in the theory of solitons. New York, LLC: Springer-Verlag, 1987.

32 KIVSHAR, Y.; LUTHER-DAVIES, B. Dark optical solitons: physics and applications. Physics Reports - Review Section of Physics Letters, v. 298, n. 2-3, p. 81-197, May 1998.

33 AGRAWAL, G. P. Nonlinear fiber optics: quantum electronics - principles and applications. Academic Press, 1089.

34 SULEM, C.; SULEM, P.-L. The nonlinear Schrödinger equation: self-focusing and wave collapse. Springer-Verlag, 1999.

35 FERREIRA, L. A.; LUCHINI, G.; ZAKRZEWSKI, W. J. The concept of quasi-integrability for modified non-linear schröedinger models. Journal of High Energy Physics, , n. 5, MAY 2011.

36 WU, T.; YANG, C.-N. Some solutions of the classical isotopic gauge field equations. 1967.

37 T'HOOFT, G. Magnetic monopoles in unified gauge theories. Nuclear Physics B, Amsterdam, v. B 79, n. 2, p. 276-284, 1974.

38 JULIA, B.; ZEE, A. Poles with both magnetic and electric charges in nonabelian gauge theory. Physics Review, v. D11, p. 2227-2232, 1975.

39 GODDARD, P.; OLIVE, D. Magnetic monopoles in gauge field-theories. Reports on Progress in Physics, v. 41, n. 9, p. 1357-1437, 1978. 
40 GIAMBIAGI, J.; ROTHE, K. Regular $N$-instanton fields and singular gauge transformations, journal $=$ Nuclear Physics $B$, year $=1977$, volume $=129$, number $=1$, pages $=111-124$, doi $=10.1016 / 0550-3213(77) 90022-0$, issn = 0550-3213, unique-id = ISI:A1977EA10900008, .

41 DE ALFARO, V.; FUBINI, S.; FURLAN, G. A new classical solution of the Yang-Mills field Equations. Physics Letters, v. B65, p. 163, 1976.

42 DE ALFARO, V.; FUBINI, S.; FURLAN, G. Properties of $O(4) \times O(2)$ symmetric solutions of the Yang-Mills field equations. Physics Letters, v. B72, p. 203, 1977.

43 DEALFARO, V.; FUBINI, S.; FURLAN, G. Classical solutions of generally invariant gauge theories. Physics Letters B, Amsterdam, v. 73, n. 4-5, p. 463-467, 1978.

44 ACTOR, A. Classical solutions of $S U(2)$ Yang-Mills theory. Reviews of Modern Physics, Woodbury, v. 51, n. 3, p. 461-525, 1979. 


\section{APPENDIX $A$}

\section{Regularisation of Wilson lines}

In order to calculate the r.h.s. of the relations (4.3.4) and (4.3.13), which give the conserved charges, as volume ordered integrals, for the Wu-Yang monopole and dyon solutions respectively, we have to evaluate the Wilson line operator $W$, defined by (2.1.1), for the connection

$$
A_{i}=-\frac{1}{e} \epsilon_{i j k} \frac{x^{j}}{r^{2}} T_{k}
$$

which has a singularity at the origin of the coordinate system. In the case of the dyon the time component of the connection is non-zero and also present a singularity at the origin. However, it does not play a role in the charge calculation since all the Wilson line operators are defined on space curves with no time component. We show in this appendix how the Wilson line operator can be regularised, when it is integrated along a purely spatial (no time) curve $\Gamma$ passing through the origin. In order to do that we shall split $\Gamma$ in three parts, $\Gamma=\Gamma_{2} \circ \tilde{\Gamma}_{\varepsilon} \circ \Gamma_{1}$, as shown in part I of the Figure (A.1).

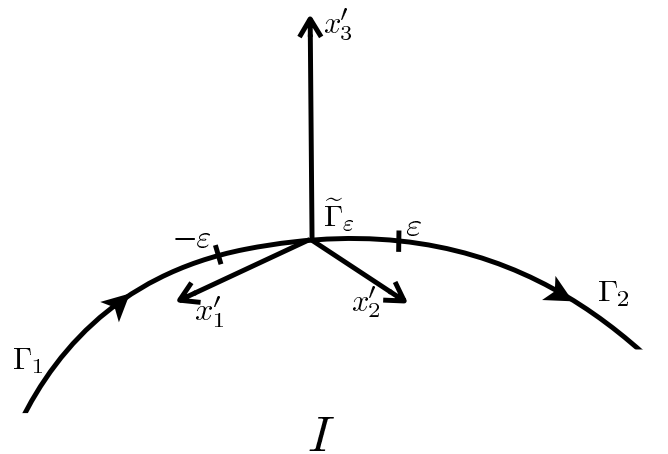

Figure A.1 - The regularisation of the Wilson line operator is done by replacing the path that passes through to the origin by a path going around it.

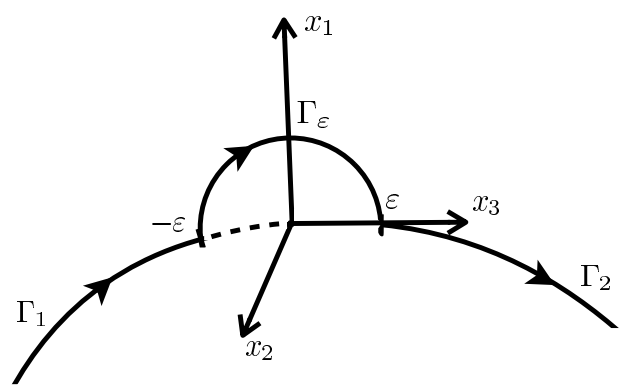

$I I$

The solution of (2.1.1) can be written as

$$
W=W_{\Gamma_{2}} \cdot W_{\tilde{\Gamma}_{\varepsilon}} \cdot W_{\Gamma_{1}}
$$

The quantities $W_{\Gamma_{1}}$ and $W_{\Gamma_{2}}$ do not involve the singularity and so we should not worry about 
them. We have to evaluate $W_{\tilde{\Gamma}_{\varepsilon}}$ which pass through the origin. We shall take $\tilde{\Gamma}_{\varepsilon}$ infinitesimally small in such a way that we can approximate it by an infinitesimal straight line of length $2 \varepsilon$ containing the origin in its middle point. Note that the quantity $A_{i} \frac{d x^{i}}{d \sigma}$, appearing in (2.1.1), is invariant under rotations, and so we can rotate the coordinate system in such a way that the $x^{3}$-axis lies parallel to $\tilde{\Gamma}_{\varepsilon}$ and in the direction of growing $\sigma$, i.e. in the sense of integration of (2.1.1), as shown in part II of the Figure A.1. Along such infinitesimal straight line $\tilde{\Gamma}_{\varepsilon}$, parametrised as $x^{3}=\sigma$, we have that $A_{i} \frac{d x^{i}}{d \sigma}=-\frac{1}{e} \frac{1}{r^{2}}\left[x^{1} T_{2}-x^{2} T_{1}\right]$. However, on $\tilde{\Gamma}_{\varepsilon}$ one has $x^{1}=x^{2}=0$, and so for $r \neq 0$ such expression vanishes. On the other hand, for $r=0$ it diverges, and so, we have a quite ill defined quantity.

In order to regularise the Wilson line we shall replace $\tilde{\Gamma}_{\varepsilon}$ by a semi-circle $\Gamma_{\varepsilon}$ of radius $\varepsilon$, with diameter being the previous straight line, and lying on the plane $x^{1} x^{3}$ as shown in part II of Figure A.1. We evaluate $W\left(\Gamma_{\varepsilon}\right)$ on such semi-circle and then take the limit $\varepsilon \rightarrow 0$. The points in $\Gamma_{\varepsilon}$ can be parametrised as

$$
x^{1}=\varepsilon \sin \sigma \quad x^{2}=0 \quad x^{3}=-\varepsilon \cos \sigma \quad 0 \leq \sigma \leq \pi
$$

Therefore for all such points we have $r=\varepsilon$, and so from (A.0.1) one has

$$
A_{i} \frac{d x^{i}}{d \sigma}=\varepsilon\left(A_{1} \cos \sigma+A_{3} \sin \sigma\right)=-\frac{1}{e} T_{2}
$$

We note that it does not depend upon $\varepsilon$ and $\sigma$, and lies in the direction of just one generator of $S U(2)$. Therefore, the problem is Abelian and the path ordering is not necessary. Then from (2.1.1) we have

$$
W\left(\Gamma_{\varepsilon}\right)=e^{i \pi T_{2}}
$$

which we take as the regularised expression for $W\left(\tilde{\Gamma}_{\varepsilon}\right)$. Of course, we would obtain different results for different choices of curves going around the origin, specially non-planar curves. However, as we will show below, the evaluation of the r.h.s of the relations (4.3.4) and (4.3.13) is independent of such choices, and the regularisation of those quantities is quite unique.

Note that for the Wu-Yang monopole and dyon solutions one has

$$
F_{i j}=\frac{1}{e} \epsilon_{i j k} \frac{n_{k}}{r^{2}} \hat{n} \cdot T ; \quad \widetilde{F}_{i j}=-\frac{\gamma}{e} \epsilon_{i j k} \frac{n_{k}}{r^{2}} \hat{n} \cdot T
$$

with $\gamma=0$ in the pure monopole case. In the evaluation of (4.3.6) and (4.3.14) we have to deal with the conjugated quantities $F_{i j}^{W}$ and $\widetilde{F}_{i j}^{W}$, and so essentially we have to worry about the quantity $W^{-1} \hat{n} \cdot T W$. Our prescription is to scan the volume (the whole space) with closed surfaces based at $x_{\mathrm{R}}$, and each of those surfaces are scanned with loops based at $x_{\mathrm{R}}$. 
The origin lies on a given surface labelled by $\zeta_{0}$, and to just one loop, labelled by $\tau_{0}$, on that surface, and corresponding to the point labelled by $\sigma_{0}$ on that loop. For the surfaces corresponding to $\zeta<\zeta_{0}$ there are no problems in the integration since everything is regular. On each loop on those surfaces $W^{-1} \hat{n} \cdot T W$ is constant and equal to $T_{\mathrm{R}}$, i.e. the value of $\hat{n} \cdot T$ at the reference point $x_{\mathrm{R}}$.

Therefore, the commutators in (4.3.6) and (4.3.14) vanish for $\zeta<\zeta_{0}$, since the conjugated tensors $F_{i j}^{W}$ and $\widetilde{F}_{i j}^{W}$ all lie in the direction of $T_{\mathrm{R}}$ on any point of any loop scanning the surfaces for $\zeta<\zeta_{0}$. On the surface for $\zeta=\zeta_{0}$ everything is fine until we reach the loop corresponding to $\tau=\tau_{0}$. In other words, the commutators in (4.3.6) and (4.3.14) also vanish for $\zeta=\zeta_{0}$ and $\tau<\tau_{0}$. Let us consider the loop corresponding to $\tau=\tau_{0}$. For $\sigma<\sigma_{0}$ we still have the vanishing of those commutators since the singularity has not been touched yet. After crossing the singularity we have that the Wilson line $W$ becomes $W_{2} W\left(\Gamma_{\varepsilon}\right) W_{1}$ (see (A.0.2)), where $W_{1}$ is the result of the integration of (2.1.1) along $\Gamma_{1}$, i.e. the curve from the reference point $x_{\mathrm{R}}$ up to the point marked $-\varepsilon$ on Figure A.1 along the loop corresponding to $\tau=\tau_{0}$, which passes through the origin. Similarly $W_{2}$ is obtained by integrating (2.1.1) along $\Gamma_{2}$, i.e. the curve from the point marked $\varepsilon$ on Figure A.1, up to some generic point beyond the origin along that same loop. In addition, $W\left(\Gamma_{\varepsilon}\right)$ is the regularised expression, given in (A.0.5), for the integration of (2.1.1) along $\tilde{\Gamma}_{\varepsilon}$.

Along the curve $\Gamma_{2}$ the connection (A.0.1) is regular, and so

$$
W_{2}^{-1} \hat{n} \cdot T W_{2}=(\hat{n} \cdot T)_{\Gamma_{2}^{0}}
$$

where $(\hat{n} \cdot T)_{\Gamma_{2}^{0}}$ is the value of $\hat{n} \cdot T$ at the initial point of the curve $\Gamma_{2}$, which is the point marked $\varepsilon$ on Figure A.1. But since we have rotated the coordinate system such that the $x^{3}$-axis lies along $\tilde{\Gamma}_{\varepsilon}$, we have that $(\hat{n} \cdot T)_{\Gamma_{2}^{0}}=T_{3}$. Now using (A.0.5), we have that

$$
W^{-1}\left(\Gamma_{\varepsilon}\right) W_{2}^{-1} \hat{n} \cdot T W_{2} W\left(\Gamma_{\varepsilon}\right)=e^{-i \pi T_{2}} T_{3} e^{i \pi T_{2}}=-T_{3}=(\hat{n} \cdot T)_{\Gamma_{1}^{\text {end }}}
$$

since $-T_{3}$ is the value of $\hat{n} \cdot T$ at the final point of the curve $\Gamma_{1}$, which is the point marked $-\varepsilon$ on Figure A.1. Along the curve $\Gamma_{1}$ the connection (A.0.1) is regular, and we have

$$
W_{1}^{-1} W^{-1}\left(\Gamma_{\varepsilon}\right) W_{2}^{-1} \hat{n} \cdot T W_{2} W\left(\Gamma_{\varepsilon}\right) W_{1}=W_{1}^{-1}(\hat{n} \cdot T)_{\Gamma_{1}^{\text {end }}} W_{1}=T_{\mathrm{R}}
$$

where $T_{\mathrm{R}}$ is the value of $(\hat{n} \cdot T)$ at the reference point $x_{\mathrm{R}}$ which is the initial point of $\Gamma_{1}$. Therefore, the field tensor and its dual, given in (A.0.6), lie in the direction of $T_{R}$ when conjugated with $W_{2} \cdot W\left(\Gamma_{\varepsilon}\right) \cdot W_{1}$, and so the commutators in (4.3.6) and (4.3.14) vanish when evaluated on the loop corresponding to $\tau=\tau_{0}$, i.e. the one passing through the singularity of 
(A.0.1). Of course, the quantities (4.3.6) and (4.3.14) will vanish on all loops scanning the surfaces for $\zeta>\zeta_{0}$, since the potential $(A .0 .1)$ is not singular there, and $W^{-1} \hat{n} \cdot T W=T_{\mathrm{R}}$, for $W$ obtained by the integration of (2.1.1) on such loops.

Consequently all the commutators in (4.3.6) and (4.3.14) vanish on any loop on the scanning of any surface on the scanning of the volume. Since the $\mathrm{Wu}$-Yang solutions have no sources we have $\tilde{J}_{123}=J_{0}=0$, and so $\mathcal{J}_{\text {monopole }}$ and $\mathcal{J}_{\text {dyon }}$ also vanishes. Therefore we conclude that the r.h.s. of (4.3.4) and (4.3.13) are equal to unity, i.e.

$$
P_{3} e^{\int_{\text {space }} d \zeta d \tau V \mathcal{J}_{\text {monopole }} V^{-1}}=\mathbb{1} ; \quad P_{3} e^{\int_{\text {space }} d \zeta d \tau V \mathcal{J}_{\text {dyon }} V^{-1}}=\mathbb{1}
$$

We now come to the issue of the uniqueness of the regularisation procedure. We have chosen to replace the segment $\tilde{\Gamma}_{\varepsilon}$ by the semi-cicle $\Gamma_{\varepsilon}$. Let us now analyze what happens to the quantity $W^{-1}\left(\Gamma_{\varepsilon}\right)(\hat{n} \cdot T)_{\Gamma_{1}^{\text {end }}} W\left(\Gamma_{\varepsilon}\right)=W^{-1}\left(\Gamma_{\varepsilon}\right)\left(-T_{3}\right) W\left(\Gamma_{\varepsilon}\right)$, when we make arbitrary infinitesimal variations on the semi-circle $\Gamma_{\varepsilon}$ keeping its end points fixed, i.e. the points marked $\varepsilon$ and $-\varepsilon$ on Figure A.1. We have

$$
\begin{aligned}
\delta\left[W^{-1}\left(\Gamma_{\varepsilon}\right)\left(-T_{3}\right) W\left(\Gamma_{\varepsilon}\right)\right] & =\left[W^{-1}\left(\Gamma_{\varepsilon}\right)\left(-T_{3}\right) W\left(\Gamma_{\varepsilon}\right), W^{-1}\left(\Gamma_{\varepsilon}\right) \delta W\left(\Gamma_{\varepsilon}\right)\right] \\
& =\left[T_{3}, W^{-1}\left(\Gamma_{\varepsilon}\right) \delta W\left(\Gamma_{\varepsilon}\right)\right]
\end{aligned}
$$

where in the last equality we have used (A.0.5) and (A.0.8). The variation of the Wilson line can be easily evaluated using for instances the techniques of section 2 of (6). When the end points of the curve $\Gamma_{\varepsilon}$ are kept fixed one gets

$$
W^{-1}\left(\Gamma_{\varepsilon}\right) \delta W\left(\Gamma_{\varepsilon}\right)=\int_{0}^{\pi} d \sigma W^{-1} F_{i j} W \frac{d x^{i}}{d \sigma} \delta x^{j}
$$

where $F_{i j}$ is the curvature, given in (A.0.6), of the connection (A.0.1), and where $W$ in the integrand in (A.0.12), is obtained by integrating (2.1.1) along $\Gamma_{\varepsilon}$, from its initial point at $\sigma=0$ to the point $\sigma=\sigma$ where the tensor $F_{i j}$ is evaluated. As long as the transformed curve does not pass through the singularity of the connection (A.0.1), the relations $D_{i}(n \cdot T)=0$ and $\frac{d}{d \sigma}\left(W^{-1} n \cdot T W\right)=0$ can be used to show that $W^{-1} \hat{n} \cdot T W=-T_{3}$, where $-T_{3}$ is the value of $\hat{n} \cdot T$ at the initial point of $\Gamma_{\varepsilon}$. Therefore, the integrand in (A.0.12) always lies in the direction of $T_{3}$, and so

$$
\delta\left[W^{-1}\left(\Gamma_{\varepsilon}\right)\left(-T_{3}\right) W\left(\Gamma_{\varepsilon}\right)\right]=0
$$

Consequently any curve $\Gamma$, with the same end points as $\Gamma_{\varepsilon}$, and that can be continuously deformed into $\Gamma_{\varepsilon}$, satisfies $W^{-1}\left(\Gamma_{\varepsilon}\right)\left(-T_{3}\right) W\left(\Gamma_{\varepsilon}\right)=W^{-1}(\Gamma)\left(-T_{3}\right) W(\Gamma)=T_{3}$. That shows that our prescription for the regularisation of the Wilson line is independent of the choice of the curve replacing the segment $\tilde{\Gamma}_{\varepsilon}$. 
Note that the special role being played by $T_{3}$ is an artifact of our choice of the orientation of the coordinate axis with respect to the curve. Note in addition that our results do not imply that the Wilson line does not change. It is just the conjugation of $T_{3}$ by the Wilson line that remains invariant. In the cases where the variation of the curve lies on the same plane as $\Gamma_{\varepsilon}$, then the Wilson line itself is invariant. The reason is that the r.h.s of (A.0.12) measures the magnetic flux through the infinitesimal surface spanned by the variation, and since the magnetic field is radial it is parallel to such surface, and so $\delta W\left(\Gamma_{\varepsilon}\right)=0$ in such cases. 


\section{APPENDIX $B$}

\section{Explicity quantities involved in equation (5.1.25)}

We give in this appendix the first few explicit expressions for the parameters $\zeta_{i}^{(-n)}, i=1,2$, introduced in (5.1.26), for the components $a_{x}$ of the connection defined in (5.1.25), and the quantities $\alpha^{(j,-n)}, j=1,2,3$, introduced in (5.1.33). On the r.h.s. of the equations below we use the following notation: (for partial derivatives w.r.t. $x$ and $t$ )

$$
\star^{(n, m)} \equiv \partial_{x}^{n} \partial_{t}^{m} \star
$$

The expressions for $\zeta_{i}^{(-n)}$ are:

$$
\begin{aligned}
\zeta_{1}^{(-1)} & =0 \\
\zeta_{2}^{(-1)} & =2 \sqrt{|\eta|} \sqrt{R}, \\
\zeta_{1}^{(-2)} & =\frac{i \sqrt{|\eta|} R^{(1,0)}}{\sqrt{R}}, \\
\zeta_{2}^{(-2)} & =\sqrt{|\eta|} \varphi^{(1,0)} \sqrt{R} \\
\zeta_{1}^{(-3)} & =\frac{i\left(\sqrt{|\eta|} \varphi^{(1,0)} R^{(1,0)}+\sqrt{|\eta|} \varphi^{(2,0)} R\right)}{\sqrt{R}} \\
\zeta_{2}^{(-3)} & =\frac{16|\eta|^{3 / 2} \sigma R^{3}+3 \sqrt{|\eta|}\left(\varphi^{(1,0)}\right)^{2} R^{2}-6 \sqrt{|\eta|} R^{(2,0)} R+3 \sqrt{|\eta|}\left(R^{(1,0)}\right)^{2}}{6 R^{3 / 2}}, \\
\zeta_{1}^{(-4)} & =\frac{i}{12 R^{5 / 2}}\left[64|\eta|^{3 / 2} \sigma R^{(1,0)} R^{3}+9 \sqrt{|\eta|}\left(\varphi^{(1,0)}\right)^{2} R^{(1,0)} R^{2}+18 \sqrt{|\eta|} \varphi^{(1,0)} \varphi^{(2,0)} R^{3}\right. \\
& \left.-12 \sqrt{|\eta|} R^{(3,0)} R^{2}+18 \sqrt{|\eta|} R^{(1,0)} R^{(2,0)} R-9 \sqrt{|\eta|}\left(R^{(1,0)}\right)^{3}\right] \\
\zeta_{2}^{(-4)} & =\frac{1}{4 R^{3 / 2}\left[16|\eta|^{3 / 2} \sigma \varphi^{(1,0)} R^{3}-6 \sqrt{|\eta|} \varphi^{(2,0)} R^{(1,0)} R-6 \sqrt{|\eta|} \varphi^{(1,0)} R^{(2,0)} R\right.} \\
& \left.+3 \sqrt{|\eta|} \varphi^{(1,0)}\left(R^{(1,0)}\right)^{2}+\sqrt{|\eta|}\left(\varphi^{(1,0)}\right)^{3} R^{2}-4 \sqrt{|\eta|} \varphi^{(3,0)} R^{2}\right]
\end{aligned}
$$


The components $a_{x}^{(3, n)}$ introduced in (??) are:

$$
\begin{aligned}
a_{x}^{(3,0)} & =\frac{1}{2} i \varphi^{(1,0)} \\
a_{x}^{(3,-1)} & =2 i|\eta| \sigma R \\
a_{x}^{(3,-2)} & =i|\eta| \sigma \varphi^{(1,0)} R \\
a_{x}^{(3,-3)} & =\frac{i|\eta|\left(4|\eta| R^{3}+\sigma\left(\varphi^{(1,0)}\right)^{2} R^{2}-2 \sigma R^{(2,0)} R+\sigma\left(R^{(1,0)}\right)^{2}\right)}{2 R} \\
a_{x}^{(3,-4)} & =\frac{i|\eta|}{4 R}\left[12|\eta| \varphi^{(1,0)} R^{3}-6 \sigma R\left(\varphi^{(2,0)} R^{(1,0)}+\varphi^{(1,0)} R^{(2,0)}\right)+3 \sigma \varphi^{(1,0)}\left(R^{(1,0)}\right)^{2}\right. \\
& \left.+\sigma\left(\left(\varphi^{(1,0)}\right)^{3}-4 \varphi^{(3,0)}\right) R^{2}\right] .
\end{aligned}
$$

The quantities $\alpha^{(j,-n)}$, introduced in (5.1.33) are:

$$
\begin{aligned}
\alpha^{(3,0)} & =1 \\
\alpha^{(3,-1)} & =0 \\
\alpha^{(3,-2)} & =2|\eta| \sigma R \\
\alpha^{(3,-3)} & =2|\eta| \sigma \varphi^{(1,0)} R \\
\alpha^{(3,-4)} & =6|\eta|^{2} R^{2}+\frac{3}{2}|\eta| \sigma\left(\varphi^{(1,0)}\right)^{2} R-2|\eta| \sigma R^{(2,0)}+\frac{3|\eta| \sigma\left(R^{(1,0)}\right)^{2}}{2 R}
\end{aligned}
$$

and

$$
\begin{aligned}
\alpha^{(1,0)} & =0 \\
\alpha^{(1,-1)} & =-2 \sqrt{|\eta|} \sqrt{R} \\
\alpha^{(1,-2)} & =-\sqrt{|\eta|} \varphi^{(1,0)} \sqrt{R}, \\
\alpha^{(1,-3)} & =-4|\eta|^{3 / 2} \sigma R^{3 / 2}-\frac{1}{2} \sqrt{|\eta|}\left(\varphi^{(1,0)}\right)^{2} \sqrt{R}-\frac{\sqrt{|\eta|}\left(R^{(1,0)}\right)^{2}}{2 R^{3 / 2}}+\frac{\sqrt{|\eta|} R^{(2,0)}}{\sqrt{R}} \\
\alpha^{(1,-4)} & =-6|\eta|^{3 / 2} \sigma \varphi^{(1,0)} R^{3 / 2}-\frac{3 \sqrt{|\eta|} \varphi^{(1,0)}\left(R^{(1,0)}\right)^{2}}{4 R^{3 / 2}}+\frac{3 \sqrt{|\eta|} \varphi^{(1,0)} R^{(2,0)}}{2 \sqrt{R}} \\
& +\frac{3 \sqrt{|\eta|} \varphi^{(2,0)} R^{(1,0)}}{2 \sqrt{R}}-\frac{1}{4} \sqrt{|\eta|}\left(\varphi^{(1,0)}\right)^{3} \sqrt{R}+\sqrt{|\eta|} \varphi^{(3,0)} \sqrt{R}
\end{aligned}
$$


and

$$
\begin{aligned}
\alpha^{(2,0)} & =0 \\
\alpha^{(2,-1)} & =0 \\
\alpha^{(2,-2)} & =-\frac{i \sqrt{|\eta|} R^{(1,0)}}{\sqrt{R}}, \\
\alpha^{(2,-3)} & =-\frac{i \sqrt{|\eta|} \varphi^{(1,0)} R^{(1,0)}}{\sqrt{R}}-i \sqrt{|\eta|} \varphi^{(2,0)} \sqrt{R} \\
\alpha^{(2,-4)} & =-6 i|\eta|^{3 / 2} \sigma \sqrt{R} R^{(1,0)}-\frac{3 i \sqrt{|\eta|}\left(\varphi^{(1,0)}\right)^{2} R^{(1,0)}}{4 \sqrt{R}}-\frac{3}{2} i \sqrt{|\eta|} \varphi^{(1,0)} \varphi^{(2,0)} \sqrt{R} \\
& +\frac{3 i \sqrt{|\eta|}\left(R^{(1,0)}\right)^{3}}{4 R^{5 / 2}}-\frac{3 i \sqrt{|\eta|} R^{(2,0)} R^{(1,0)}}{2 R^{3 / 2}}+\frac{i \sqrt{|\eta|} R^{(3,0)}}{\sqrt{R}}
\end{aligned}
$$




\section{APPENDIX C}

\section{The Hirota solutions}

Here we construct the one and two bright soliton solutions of the integrable NLS theory (5.1.10) using the Hirota method. The one and two dark soliton solutions require a different procedure from the one described here. We introduce the Hirota tau-functions as

$$
\psi_{0}=\frac{i}{\gamma} \frac{\tau_{+}}{\tau_{0}} ; \quad \bar{\psi}_{0}=-\frac{i}{\bar{\gamma}} \frac{\tau_{-}}{\tau_{0}}
$$

where $\eta=\gamma \bar{\gamma}$. The bright soliton solutions exist for $\eta<0$ and so we need $\bar{\gamma}=-\gamma^{*}$, and then $\frac{\tau_{-}}{\tau_{0}}=-\left(\frac{\tau_{+}}{\tau_{0}}\right)^{*}$. Putting (…1) into the the NLS equation (5.1.10) and its complex conjugate we get the two Hirota equations

$$
\begin{aligned}
& \tau_{0}^{2}\left(i \partial_{t} \tau_{+}+\partial_{x}^{2} \tau_{+}\right)-2 \tau_{0} \partial_{x} \tau_{+} \partial_{x} \tau_{0}-2 \tau_{+}^{2} \tau_{-}- \\
& \tau_{0} \tau_{+}\left(i \partial_{t} \tau_{0}+\partial_{x}^{2} \tau_{0}\right)+2 \tau_{+}\left(\partial_{x} \tau_{0}\right)^{2}=0 \\
& \tau_{0}^{2}\left(-i \partial_{t} \tau_{-}+\partial_{x}^{2} \tau_{-}\right)-2 \tau_{0} \partial_{x} \tau_{-} \partial_{x} \tau_{0}-2 \tau_{-}^{2} \tau_{+}- \\
& \tau_{0} \tau_{-}\left(-i \partial_{t} \tau_{0}+\partial_{x}^{2} \tau_{0}\right)+2 \tau_{-}\left(\partial_{x} \tau_{0}\right)^{2}=0 .
\end{aligned}
$$

The one-soliton solution of (C.0.2) is given by

$$
\begin{aligned}
\tau_{0} & =1+a_{+} a_{-} \frac{z_{1} z_{2}}{\left(z_{1}-z_{2}\right)^{2}} e^{i \Gamma\left(z_{1}\right)} e^{-i \Gamma\left(z_{2}\right)}, \\
\tau_{+} & =a_{-} z_{2} e^{-i \Gamma\left(z_{2}\right)} \\
\tau_{-} & =a_{+} z_{1} e^{i \Gamma\left(z_{1}\right)}
\end{aligned}
$$

with $a_{ \pm}, z_{1}$ and $z_{2}$ being complex parameters and $\Gamma\left(z_{i}\right)=z_{i}^{2} t-z_{i} x$. We choose $z_{2}=z_{1}^{*}$ and $a_{-}=-a_{+}^{*}$, which implies that $\tau_{-}=-\tau_{+}^{*}$, and $\tau_{0}$ is real. We then parametrize them as $a_{ \pm}=i a e^{ \pm i \theta}, \quad z_{1}=\frac{v}{2}+i \rho=\sqrt{\frac{v^{2}}{4}+\rho^{2}} e^{i \zeta}, \quad \gamma=i \sqrt{|\eta|} e^{i \phi}, \quad \bar{\gamma}=i \sqrt{|\eta|} e^{-i \phi}$ 
with $a>0$, and $v$ and $\rho$ both real. We replace $a$ by $x_{0}$ defined as

$$
a \frac{\sqrt{\frac{v^{2}}{4}+\rho^{2}}}{2|\rho|}=e^{-\rho x_{0}}
$$

and find from (C.0.1) that

$$
\psi_{0}=\frac{i e^{-i(\theta+\zeta+\phi)}}{\sqrt{|\eta|}}|\rho| \frac{e^{i\left[\left(\rho^{2}-\frac{v^{2}}{4}\right) t+\frac{v}{2} x\right]}}{\cosh \left[\rho\left(x-v t-x_{0}\right)\right]} .
$$

This expression, up to an overall constant phase factor (due to the symmetry (5.1.8)) is the one-bright-soliton given in (5.1.11).

The two-soliton solution of (C.0.2) is given by

$$
\begin{aligned}
\tau_{0} & =1+a_{+} a_{-} \frac{z_{1} z_{2}}{\left(z_{1}-z_{2}\right)^{2}} e^{i \Gamma\left(z_{1}\right)} e^{-i \Gamma\left(z_{2}\right)}+b_{+} b_{-} \frac{w_{1} w_{2}}{\left(w_{1}-w_{2}\right)^{2}} e^{i \Gamma\left(w_{1}\right)} e^{-i \Gamma\left(w_{2}\right)} \\
& +a_{+} b_{-} \frac{z_{1} w_{2}}{\left(z_{1}-w_{2}\right)^{2}} e^{i \Gamma\left(z_{1}\right)} e^{-i \Gamma\left(w_{2}\right)}+a_{-} b_{+} \frac{w_{1} z_{2}}{\left(w_{1}-z_{2}\right)^{2}} e^{-i \Gamma\left(z_{2}\right)} e^{i \Gamma\left(w_{1}\right)} \\
& +a_{+} a_{-} b_{+} b_{-} \frac{z_{1} z_{2} w_{1} w_{2}\left(z_{1}-w_{1}\right)^{2}\left(z_{2}-w_{2}\right)^{2}}{\left(z_{1}-z_{2}\right)^{2}\left(w_{1}-w_{2}\right)^{2}\left(z_{1}-w_{2}\right)^{2}\left(w_{1}-z_{2}\right)^{2}} e^{i \Gamma\left(z_{1}\right)} e^{-i \Gamma\left(z_{2}\right)} e^{i \Gamma\left(w_{1}\right)} e^{-i \Gamma\left(w_{2}\right)}, \\
\tau_{+} & =a_{-} z_{2} e^{-i \Gamma\left(z_{2}\right)}+b_{-} w_{2} e^{-i \Gamma\left(w_{2}\right)}+a_{+} a_{-} b_{-} \frac{w_{2} z_{1} z_{2}\left(w_{2}-z_{2}\right)^{2}}{\left(w_{2}-z_{1}\right)^{2}\left(z_{1}-z_{2}\right)^{2}} e^{i \Gamma\left(z_{1}\right)} e^{-i \Gamma\left(z_{2}\right)} e^{-i \Gamma\left(w_{2}\right)} \\
& +a_{-} b_{+} b_{-} \frac{w_{1} w_{2} z_{2}\left(w_{2}-z_{2}\right)^{2}}{\left(w_{1}-w_{2}\right)^{2}\left(w_{1}-z_{2}\right)^{2}} e^{-i \Gamma\left(z_{2}\right)} e^{i \Gamma\left(w_{1}\right)} e^{-i \Gamma\left(w_{2}\right)}, \\
\tau_{-} & =a_{+} z_{1} e^{i \Gamma\left(z_{1}\right)}+b_{+} w_{1} e^{i \Gamma\left(w_{1}\right)}+a_{+} a_{-} b_{+} \frac{w_{1} z_{1} z_{2}\left(w_{1}-z_{1}\right)^{2}}{\left(w_{1}-z_{2}\right)^{2}\left(z_{1}-z_{2}\right)^{2}} e^{i \Gamma\left(z_{1}\right)} e^{i \Gamma\left(w_{1}\right)} e^{-i \Gamma\left(z_{2}\right)} \\
& +a_{+} b_{+} b_{-} \frac{w_{1} w_{2} z_{1}\left(w_{1}-z_{1}\right)^{2}}{\left(w_{1}-w_{2}\right)^{2}\left(w_{2}-z_{1}\right)^{2}} e^{i \Gamma\left(z_{1}\right)} e^{i \Gamma\left(w_{1}\right)} e^{-i \Gamma\left(w_{2}\right)}
\end{aligned}
$$

where $a_{ \pm}, b_{ \pm}, z_{1}, z_{2}, w_{1}$ and $w_{2}$ are arbitrary complex parameters, and as before, $\Gamma\left(w_{i}\right)=$ $w_{i}^{2} t-w_{i} x$. The two-bright-soliton solution of the NLS theory (5.1.10), corresponding to $\eta<0$, is obtained by taking $\tau_{-}=-\tau_{+}^{*}$, and $\tau_{0}$ real. One way of getting this involves putting

$$
z_{2}=z_{1}^{*}, \quad w_{2}=w_{1}^{*}, \quad a_{-}=-a_{+}^{*}, \quad b_{-}=-b_{+}^{*}
$$

and then parametrizing them as

$$
a_{ \pm}=i a_{1} e^{ \pm i \theta_{1}}, \quad b_{ \pm}=i a_{2} e^{ \pm i \theta_{2}}, \quad \gamma=i \sqrt{|\eta|} e^{i \phi}, \quad \bar{\gamma}=i \sqrt{|\eta|} e^{-i \phi}
$$

with $a_{i}>0, i=1,2$, and

$$
z_{1}=\frac{v_{1}}{2}+i \rho_{1}=\sqrt{\frac{v_{1}^{2}}{4}+\rho_{1}^{2}} e^{i \zeta_{1}}, \quad w_{1}=\frac{v_{2}}{2}+i \rho_{2}=\sqrt{\frac{v_{2}^{2}}{4}+\rho_{2}^{2}} e^{i \zeta_{2}} .
$$


This gives us

$$
\begin{aligned}
\Gamma\left(z_{1}\right) & =z_{1}^{2} t-z_{1} x=\left(\frac{v_{1}^{2}}{4}-\rho_{1}^{2}\right) t-\frac{v_{1}}{2} x-i \rho_{1}\left(x-v_{1} t\right), \\
\Gamma\left(w_{1}\right) & =w_{1}^{2} t-w_{1} x=\left(\frac{v_{2}^{2}}{4}-\rho_{2}^{2}\right) t-\frac{v_{2}}{2} x-i \rho_{2}\left(x-v_{2} t\right) .
\end{aligned}
$$

Finally, we replace $a_{i}$ by $x_{i}^{(0)}, i=1,2$, defined as

$$
a_{i} \frac{\sqrt{\frac{v_{i}^{2}}{4}+\rho_{i}^{2}}}{2\left|\rho_{i}\right|}=e^{-\rho_{i} x_{i}^{(0)}}
$$

Putting all these expressions into (C.0.7) and into (C.0.1) we obtain the final form of the two-bright-soliton solution:

$$
\begin{aligned}
\psi_{0} & =\frac{i 2 e^{-i \phi}}{\sqrt{|\eta|}} \times \\
\times & {\left[\frac{W_{1} e^{X_{1}}+W_{2} e^{X_{2}}+\frac{\Lambda_{-}}{\Lambda_{+}} e^{\left(X_{1}+X_{2}\right)}\left[W_{2} e^{X_{1}} e^{-i 2\left(\delta_{+}+\delta_{-}\right)}+W_{1} e^{X_{2}} e^{i 2\left(\delta_{+}-\delta_{-}\right)}\right]}{1+e^{2 X_{1}}+e^{2 X_{2}}+\left[\frac{\Lambda_{-}}{\Lambda_{+}}\right]^{2} e^{2\left(X_{1}+X_{2}\right)}-32 \frac{\left|\rho_{1}\right|\left|\rho_{2}\right|}{\Lambda_{+}} \cos \left(\Omega_{1}-\Omega_{2}-2 \delta_{+}\right) e^{\left(X_{1}+X_{2}\right)}}\right], }
\end{aligned}
$$

where

$$
\Lambda_{ \pm}=\left(v_{1}-v_{2}\right)^{2}+4\left(\rho_{1} \pm \rho_{2}\right)^{2} ; \quad \delta_{ \pm}=\operatorname{ArcTan}\left[\frac{2\left(\rho_{1} \pm \rho_{2}\right)}{\left(v_{1}-v_{2}\right)}\right]
$$

and

$$
W_{i}=\left|\rho_{i}\right| e^{-i \Omega_{i}}
$$

with

$$
\Omega_{i}=\left(\frac{v_{i}^{2}}{4}-\rho_{i}^{2}\right) t-\frac{v_{i}}{2} x+\theta_{i}+\zeta_{i}, \quad X_{i}=\rho_{i}\left(x-v_{i} t-x_{i}^{(0)}\right) \quad i=1,2 .
$$

